REPRESENTATION THEORY

An Electronic Journal of the American Mathematical Society

Volume 14, Pages 379-416 (May 6, 2010)

S 1088-4165(10)00375-4

\title{
CYCLOTOMIC $q$-SCHUR ALGEBRAS ASSOCIATED TO THE ARIKI-KOIKE ALGEBRA
}

\author{
TOSHIAKI SHOJI AND KENTARO WADA
}

\begin{abstract}
Let $\mathcal{H}_{n, r}$ be the Ariki-Koike algebra associated to the complex reflection group $\mathfrak{S}_{n} \ltimes(\mathbb{Z} / r \mathbb{Z})^{n}$, and let $\mathcal{S}(\Lambda)$ be the cyclotomic $q$-Schur algebra associated to $\mathcal{H}_{n, r}$, introduced by Dipper, James and Mathas. For each $\mathbf{p}=\left(r_{1}, \ldots, r_{g}\right) \in \mathbb{Z}_{>0}^{g}$ such that $r_{1}+\cdots+r_{g}=r$, we define a subalgebra $\mathcal{S}^{\mathbf{p}}$ of $\mathcal{S}(\Lambda)$ and its quotient algebra $\overline{\mathcal{S}}^{\mathbf{p}}$. It is shown that $\mathcal{S}^{\mathbf{p}}$ is a standardly based algebra and $\overline{\mathcal{S}}^{\mathbf{p}}$ is a cellular algebra. By making use of these algebras, we prove a product formula for decomposition numbers of $\mathcal{S}(\Lambda)$, which asserts that certain decomposition numbers are expressed as a product of decomposition numbers for various cyclotomic $q$-Schur algebras associated to ArikiKoike algebras $\mathcal{H}_{n_{i}, r_{i}}$ of smaller rank. This is a generalization of the result of N. Sawada. We also define a modified Ariki-Koike algebra $\overline{\mathcal{H}}^{\mathbf{p}}$ of type $\mathbf{p}$, and prove the Schur-Weyl duality between $\overline{\mathcal{H}}^{\mathbf{p}}$ and $\overline{\mathcal{S}}^{\mathbf{p}}$.
\end{abstract}

\section{INTRODUCTION}

Let $\mathcal{H}=\mathcal{H}_{n, r}$ be the Ariki-Koike algebra over an integral domain $R$ associated to the complex reflection group $W_{n, r}=\mathfrak{S}_{n} \ltimes(\mathbb{Z} / r \mathbb{Z})^{n}$ with parameters $q, Q_{1}, \ldots, Q_{r} \in$ $R$ such that $q$ is a unit in $R$. Let $\widetilde{\mathcal{P}}_{n, r}$ (resp. $\mathcal{P}_{n, r}$ ) be the set of $r$-compositions (resp. $r$-partitions) of $n$. The cyclotomic $q$-Schur algebra $\mathcal{S}(\Lambda)$ associated to $\mathcal{H}$ was introduced by Dipper, James and Mathas [DJM], which is the endomorphism algebra of a certain $\mathcal{H}$-module $M=\bigoplus_{\mu \in \Lambda} M^{\mu}$, where $\Lambda$ is a saturated subset of $\widetilde{\mathcal{P}}_{n, r}$. They showed that $\mathcal{S}(\Lambda)$ is a cellular algebra in the sense of Graham and Lehrer [GL, and Mathas [M] showed that the Schur-Weyl duality (i.e., the double centralizer property) holds between $\mathcal{H}$ and $\mathcal{S}(\Lambda)$ in the case where $\Lambda=\widetilde{\mathcal{P}}_{n, r}$ with a certain condition.

On the other hand, the modified Ariki-Koike algebra $\overline{\mathcal{H}}$ was introduced in [SawS], under the condition that $\left(^{*}\right)$ " $Q_{i}-Q_{j}$ are units in $R$ for each $i \neq j$ ", based on the study of the Schur-Weyl duality between $\mathcal{H}$ and a certain subalgebra of the quantum group of type $A$ ( $\underline{\operatorname{SakS}}, \underline{\underline{S h}})$. By using the cellular structure of $\overline{\mathcal{H}}$, a cyclotomic $q$-Schur algebra associated to $\overline{\mathcal{H}}$ was constructed, in analogy to $\mathcal{S}(\Lambda)$. It was shown in $[\mathrm{SawS}$, that this cyclotomic $q$-Schur algebra is isomorphic to the quotient algebra $\overline{\mathcal{S}}^{0}$ of a certain subalgebra $\mathcal{S}^{0}$ of $\mathcal{S}(\Lambda)$, and that the Schur-Weyl duality holds between $\overline{\mathcal{H}}$ and $\overline{\mathcal{S}}^{0}$. Moreover, the structure theorem for $\overline{\mathcal{S}}^{0}$ was proved, which asserts that $\overline{\mathcal{S}}^{0}$ is a direct sum of tensor products of various $q$-Schur algebras $\mathcal{S}\left(\widetilde{\mathcal{P}}_{n_{i}, 1}\right)$ associated to the Iwahori-Hecke algebra of type $A_{n_{i}-1}$.

Received by the editors November 1, 2007 and, in revised form, February 6, 2010.

2010 Mathematics Subject Classification. Primary 20C08, 20G43.

(C)2010 American Mathematical Society 
In $\mathrm{Sa}$, N. Sawada reconstructed the subalgebra $\mathcal{S}^{0}$ of $\mathcal{S}(\Lambda)$ and its quotient $\overline{\mathcal{S}}^{0}$ based on the cellular structure on $\mathcal{S}(\Lambda)$, which works without the assumption $\left({ }^{*}\right)$. He proved that $\mathcal{S}^{0}$ is a standardly based algebra in the sense of Du and Rui [DR], and showed, in the case where $R$ is a field, that the decomposition number $d_{\lambda \mu}$ between the Weyl module $W^{\lambda}$ and the irreducible module $L^{\mu}$ of $\mathcal{S}(\Lambda)\left(\lambda, \mu \in \mathcal{P}_{n, r}\right)$ coincides with the corresponding decomposition number for $\overline{\mathcal{S}}^{0}$ whenever $\left|\lambda^{(i)}\right|=$ $\left|\mu^{(i)}\right|$ for $i=1, \ldots, r$. This implies in the case where $\Lambda=\widetilde{\mathcal{P}}_{n, r}$, under the condition $\left(^{*}\right)$, that $d_{\lambda \mu}$ can be written as a product of $d_{\lambda^{(i)} \mu^{(i)}}$ for $i=1, \ldots, r$, where $d_{\lambda^{(i)} \mu^{(i)}}$ is the decomposition number of the $q$-Schur algebra $\mathcal{S}\left(\widetilde{\mathcal{P}}_{n_{i}, 1}\right)$ with $\left|\lambda^{(i)}\right|=\left|\mu^{(i)}\right|=n_{i}$.

The subalgebra $\mathcal{S}^{0}$ is regarded, in some sense, as a Borel type subalgebra of $\mathcal{S}(\Lambda)$. For example, we have $\mathcal{S}(\Lambda)=\mathcal{S}^{0} \cdot\left(\mathcal{S}^{0}\right)^{*}$, where $\left(\mathcal{S}^{0}\right)^{*}$ is the image of $\mathcal{S}^{0}$ under the involution $*$ of $\mathcal{S}(\Lambda)$, and $\overline{\mathcal{S}}^{0}$ is a quotient of both $\mathcal{S}^{0}$ and $\left(\mathcal{S}^{0}\right)^{*}$. Thus $\overline{\mathcal{S}}^{0}$ corresponds to a Cartan subalgebra. In this paper, we consider a parabolic analogue of $\mathcal{S}^{0}$ and $\overline{\mathcal{S}}^{0}$. We fix $\mathbf{p}=\left(r_{1}, \ldots, r_{g}\right) \in \mathbb{Z}_{>0}^{g}$ such that $r_{1}+\cdots r_{g}=r$. According to $\mathbf{p}$, we regard an $r$-partition $\lambda=\left(\lambda^{(1)}, \ldots, \lambda^{(r)}\right)$ as a $g$-tuple of multi-partitions $\lambda=\left(\lambda^{[1]}, \ldots, \lambda^{[g]}\right)$, where $\lambda^{[1]}=\left(\lambda^{(1)}, \ldots, \lambda^{\left(r_{1}\right)}\right), \lambda^{[2]}=\left(\lambda^{\left(r_{1}+1\right)}, \ldots, \lambda^{\left(r_{1}+r_{2}\right)}\right)$, and so on. For example, $\lambda^{[i]}=\lambda^{(i)}$ for $i=1, \ldots, r$ if $\mathbf{p}=\left(1^{r}\right)$ with $g=r$, and $\lambda^{[1]}=\lambda$ if $\mathbf{p}=(r)$ with $g=1$. For each $\mathbf{p}$, we define a subalgebra $\mathcal{S}^{\mathbf{p}}$ of $\mathcal{S}(\Lambda)$, and its quotient algebra $\overline{\mathcal{S}}^{\mathbf{p}}$. The algebra $\mathcal{S}^{\mathbf{p}}$ coincides with $\mathcal{S}^{0}$ if $\mathbf{p}=\left(1^{r}\right)$, and coincides with $\mathcal{S}(\Lambda)$ if $\mathbf{p}=(r)$. Thus $\mathcal{S}^{\mathbf{p}}$ is a generalization of $\mathcal{S}^{0}$, and is regarded as an intermediate object between $\mathcal{S}(\Lambda)$ and $\mathcal{S}^{0}$.

All of the results in $\mathrm{Sa}$ can be generalized to our cases; $\mathcal{S}^{\mathbf{p}}$ is a standardly based algebra and $\overline{\mathcal{S}}^{\mathbf{p}}$ is a cellular algebra. Assume that $R$ is a field. For $\lambda=$ $\left(\lambda^{[1]}, \ldots, \lambda^{[g]}\right), \mu=\left(\mu^{[1]}, \ldots, \mu^{[g]}\right) \in \mathcal{P}_{n, r}$ such that $\left|\lambda^{[i]}\right|=\left|\mu^{[i]}\right|$ for $i=1, \ldots, g$, one can show (Theorem 3.13) that the decomposition number $d_{\lambda \mu}$ coincides with the corresponding decomposition number in the algebra $\overline{\mathcal{S}}^{\mathbf{p}}$. In the case where $\Lambda=\widetilde{\mathcal{P}}_{n, r}$, we prove the structure theorem (Theorem 4.15 ) for $\overline{\mathcal{S}}^{\mathrm{p}}$, which asserts that $\overline{\mathcal{S}}^{\mathbf{p}}$ is a direct sum of tensor products of various $\mathcal{S}\left(\widetilde{\mathcal{P}}_{n_{i}, r_{i}}\right)$. We remark, in contrast to the argument in [SawS], that no assumptions on parameters are required in this proof. Combining with the previous results, we obtain the product formula for decomposition numbers, namely, $d_{\lambda \mu}$ coincides with the product of $d_{\lambda^{[i]} \mu^{[i]}}$ for $i=1, \ldots, g$, where $d_{\lambda^{[i]} \mu^{[i]}}$ is the decomposition number for $\mathcal{S}\left(\widetilde{\mathcal{P}}_{n_{i}, r_{i}}\right)$ with $\left|\lambda^{[i]}\right|=$ $\left|\mu^{[i]}\right|=n_{i}$, which holds without any restriction on the parameters (Theorem 4.17).

By making use of the Schur functors on $\mathcal{S}(\Lambda)$, one can define a modified ArikiKoike algebra $\overline{\mathcal{H}}^{\mathbf{p}}$ of type $\mathbf{p}$ as a certain subalgebra of $\mathcal{S}^{\mathbf{p}}$. The algebra $\overline{\mathcal{H}}^{\mathbf{p}}$ is isomorphic to $\overline{\mathcal{H}}$ if $\mathbf{p}=\left(1^{r}\right)$, and coincides with $\mathcal{H}$ if $\mathbf{p}=(r)$. Put $Q_{i}^{\mathbf{p}}=Q_{r_{1}+\cdots+r_{i}}$ for $i=1, \ldots, g$. Under the assumption $(* *)$ " $Q_{i}^{\mathbf{p}}-Q_{j}^{\mathbf{p}}$ are units in $R$ for each $i \neq j$ ", we give a presentation of $\overline{\mathcal{H}}^{\mathbf{p}}$ which is a generalization of the presentation of $\overline{\mathcal{H}}$ given in SawS. We show that $\overline{\mathcal{S}}^{\mathbf{p}}$ is realized as an endomorphism algebra of a certain $\overline{\mathcal{H}}^{\mathbf{p}}$-module $\bar{M}^{\mathbf{p}}=\bigoplus_{\mu \in \Lambda} \bar{M}^{\mu}$, and prove the Schur-Weyl duality between $\overline{\mathcal{S}}^{\mathbf{p}}$ and $\overline{\mathcal{H}}^{\mathbf{p}}$. In the case where the parameters $q, Q_{1}, \ldots, Q_{r}$ satisfy the separation condition in the sense of $\left[\mathrm{A}\right.$ ] (see (8.3.1)), it is shown that all the $\overline{\mathcal{H}}^{\mathbf{p}}$ are isomorphic to $\mathcal{H}$, and so the above results give new presentations of $\mathcal{H}$.

By using the Jantzen filtration, $v$-decomposition numbers $d_{\lambda \mu}(v)$ for $\mathcal{S}(\Lambda)$ can be defined, which is a polynomial analogue of $d_{\lambda \mu}$. The results in this paper concerning the decomposition numbers for $\mathcal{S}(\Lambda), \mathcal{S}^{\mathbf{p}}, \overline{\mathcal{S}}^{\mathbf{p}}$ are generalized to $v$-decomposition 
numbers. In particular, the product formula for $v$-decomposition numbers is obtained, which is discussed in $[\mathrm{W}$.

Notation. Let $R$ be an integral domain and $M$ a free $R$-module of finite rank. We denote by End $M$ the endomorphism algebra of $M$, where the composition is defined by $(f \circ g)(m)=f(g(m))$ for $f, g \in$ End $M, m \in M$. Thus End $M$ acts on $M$ from the left by $(f, m) \mapsto f(m)$. We denote by $\operatorname{End}^{0} M$ the opposite algebra of End $M$. If an $R$-algebra $A$ (resp. $B$ ) acts on $M$ from the left (resp. from the right), then we have a natural homomorphism of $R$-algebras $A \rightarrow$ End $M$ (resp. $B \rightarrow \operatorname{End}^{0} M$ ). If an $R$-algebra $X$ acts on $M$ from the right or left, we denote by End $_{X} M$ the subalgebra of End $M$ consisting of endomorphisms commuting with $X$. The subalgebra $\operatorname{End}_{X}^{0} M$ of $\operatorname{End}^{0} M$ is defined similarly.

\section{Recollection of Cyclotomic $q$-Schur algebras}

1.1. Let $R$ be an integral domain, $q, Q_{1}, \ldots, Q_{r}$ be elements in $R$ with $q$ invertible. The Ariki-Koike algebra $\mathcal{H}=\mathcal{H}_{n, r}$ associated to the complex reflection group $\mathfrak{S}_{n} \ltimes(\mathbb{Z} / r \mathbb{Z})^{n}$ is an associative algebra over $R$ with generators $T_{0}, T_{1}, \ldots, T_{n-1}$ subject to the condition

$$
\begin{array}{ll}
\left(T_{0}-Q_{1}\right) \cdots\left(T_{0}-Q_{r}\right)=0, & \\
\left(T_{i}-q\right)\left(T_{i}+q^{-1}\right)=0 & \\
T_{0} T_{1} T_{0} T_{1}=T_{1} T_{0} T_{1} T_{0}, & \\
T_{i} T_{j}=T_{j} T_{i} & (|i-j| \geq 2), \\
T_{i} T_{i+1} T_{i}=T_{i+1} T_{i} T_{i+1} & (1 \leq i \leq n-2) .
\end{array}
$$

It is known that $\mathcal{H}$ is a free $R$-module with rank $n ! r^{n}$. We denote by $\mathcal{H}_{n}$ the subalgebra of $\mathcal{H}$ generated by $T_{1}, \ldots, T_{n-1}$, which is isomorphic to the IwahoriHecke algebra associated to the symmetric group $\mathfrak{S}_{n}$ of degree $n$.

1.2. It is known by [DJM] that $\mathcal{H}$ has a structure of the cellular algebra. In order to describe the cellular basis of $\mathcal{H}$, we prepare some notation. An element $\mu=$ $\left(\mu_{1}, \ldots, \mu_{m}\right) \in \mathbb{Z}_{\geq 0}^{m}$ is called a composition of length $\leq m$, and $|\mu|=\sum \mu_{i}$ is called the size of $\mu$. An $r$-composition $\lambda=\left(\lambda^{(1)}, \ldots, \lambda^{(r)}\right)$ is an $r$-tuple of compositions $\lambda^{(i)}=\left(\lambda_{1}^{(i)}, \ldots, \lambda_{m_{i}}^{(i)}\right)$. The size $|\lambda|$ of $\lambda$ is defined by $|\lambda|=\sum_{i=1}^{r}\left|\lambda^{(i)}\right|$. We denote by $\lambda$ by $\lambda=\left(\lambda_{j}^{(i)}\right)$. A composition $\mu=\left(\mu_{1}, \ldots, \mu_{m}\right)$ is called a partition if $\mu_{1} \geq \cdots \geq$ $\mu_{m} \geq 0$. An $r$-composition $\lambda$ is called an $r$-partition if $\lambda^{(i)}$ is a partition for all $i$. We fix $\mathbf{m}=\left(m_{1}, \ldots, m_{r}\right) \in \mathbb{Z}_{>0}^{r}$ once and for all, and denote by $\widetilde{\mathcal{P}}_{n, r}=\widetilde{\mathcal{P}}_{n, r}(\mathbf{m})$ the set of $r$-compositions $\lambda=\left(\lambda^{(1)}, \ldots, \lambda^{(r)}\right)$ of size $n$ such that $\lambda^{(i)}$ is a composition of length $\leq m_{i}$. Similarly, we define the set $\mathcal{P}_{n, r}=\mathcal{P}_{n, r}(\mathbf{m})$ of $r$-partitions. If $m_{i} \geq n$ for any $i, \mathcal{P}_{n, r}(\mathbf{m})$ are mutually identified for all $\mathbf{m}$. However, even in that case, $\widetilde{\mathcal{P}}_{n, r}(\mathbf{m})$ depends on the choice of $\mathbf{m}$.

For $r$-compositions $\lambda=\left(\lambda_{j}^{(i)}\right)$ and $\mu=\left(\mu_{j}^{(i)}\right)$, we define a dominance order $\lambda \unrhd \mu$ by the condition

$$
\sum_{c=1}^{k-1}\left|\lambda^{(c)}\right|+\sum_{j=1}^{i}\left|\lambda_{j}^{(k)}\right| \geq \sum_{c=1}^{k-1}\left|\mu^{(c)}\right|+\sum_{j=1}^{i}\left|\mu_{j}^{(k)}\right|
$$

for any $1 \leq k \leq r$ and $1 \leq i \leq m_{k}$. If $\lambda \unrhd \mu$ and $\lambda \neq \mu$, we write it as $\lambda \triangleright \mu$. 
Let $\lambda$ be an $r$-partition of $n$. We identify $\lambda$ with the $r$-tuple of Young diagrams, and refer it as the Young diagram of $\lambda$. We denote by $\operatorname{Std}(\lambda)$ the set of standard tableau $\mathfrak{t}=\left(\mathfrak{t}^{(1)}, \ldots, \mathfrak{t}^{(r)}\right)$ of shape $\lambda$, i.e., $\mathfrak{t}$ is a Young diagram of $\lambda$ with letters $1, \ldots, n$ attached to the nodes of the diagram, under the condition that $\mathfrak{t}^{(i)}$ is a standard tableau in the usual sense for each $i$. We define $\mathfrak{t}^{\lambda} \in \operatorname{Std}(\lambda)$ by attaching the letters $1,2, \ldots, n$ to the nodes of the Young diagram $\lambda$ in this order, from left to right, and from top to bottom for $\mathfrak{t}^{(1)}$, and then for $\mathfrak{t}^{(2)}$, and so on. $\mathfrak{S}_{n}$ acts naturally on the set of tableaux from the right, and we denote by $d(\mathfrak{t})$ the element in $\mathfrak{S}_{n}$ such that $\mathfrak{t}=\mathfrak{t}^{\lambda} d(\mathfrak{t})$ for each $\mathfrak{t} \in \operatorname{Std}(\lambda)$. More generally, the set $\mathrm{r}-\operatorname{Std}(\mu)$ of row-standard tableaux of shape $\mu$ is defined for $\mu \in \widetilde{\mathcal{P}}_{n, r}$, by replacing a standard tableau $\mathfrak{t}^{(i)}$ by a row-standard tableau. Then $\mathfrak{t}^{\mu}$ is defined similarly, and $d(\mathfrak{t}) \in \mathfrak{S}_{n}$ is defined also for $\mathfrak{t} \in \mathrm{r}-\operatorname{Std}(\mu)$.

For $\mu \in \widetilde{\mathcal{P}}_{n, r}$, we define $r$-tuples of integers

$$
\alpha(\mu)=\left(\alpha_{1}, \ldots, \alpha_{r}\right), \quad \mathbf{a}(\mu)=\left(a_{1}, \ldots, a_{r}\right)
$$

by $\alpha_{i}=\left|\mu^{(i)}\right|$, and $a_{i}=\sum_{j=1}^{i-1}\left|\mu^{(j)}\right|$ for $i=1, \ldots, r$. (Note that $a_{1}=0$.)

We define $L_{k} \in \mathcal{H}$ by $L_{k}=T_{k-1} \cdots T_{1} T_{0} T_{1} \cdots T_{k-1}$ for $k=1, \ldots, n$. Then $L_{1}, \ldots, L_{n}$ commute with each other. For $\mathbf{a}=\left(a_{1}, \ldots, a_{r}\right) \in \mathbb{Z}_{\geq 0}^{r}$, we define $u_{\mathbf{a}}^{+} \in \mathcal{H}$ by $u_{\mathbf{a}}^{+}=u_{\mathbf{a}, 1} u_{\mathbf{a}, 2} \cdots u_{\mathbf{a}, r}$, where

$$
u_{\mathbf{a}, k}=\prod_{i=1}^{a_{k}}\left(L_{i}-Q_{k}\right) .
$$

For $\mu \in \widetilde{\mathcal{P}}_{n, r}$, let $\mathfrak{S}_{\mu}=\mathfrak{S}_{\mu^{(1)}} \times \cdots \times \mathfrak{S}_{\mu^{(r)}}$ be the Young subgroup of $\mathfrak{S}_{n}$. We define $x_{\mu} \in \mathcal{H}_{n}$ by $x_{\mu}=\sum_{w \in \mathfrak{S}_{\mu}} q^{l(w)} T_{w}$, where $l(w)$ is the length of $w \in \mathfrak{S}_{n}$, and $T_{w}$ is a basis element of $\mathcal{H}_{n}$ corresponding to $w \in \mathfrak{S}_{n}$. We define $m_{\mu} \in \mathcal{H}$ by $m_{\mu}=u_{\mathbf{a}}^{+} x_{\mu}=$ $x_{\mu} u_{\mathbf{a}}^{+}$. For $\mathfrak{s}, \mathfrak{t} \in \mathrm{r}-\operatorname{Std}(\mu)$, we define $m_{\mathfrak{s t}} \in \mathcal{H}$ by $m_{\mathfrak{s t}}=T_{d(\mathfrak{s})}^{*} m_{\mu} T_{d(\mathfrak{t})}$, where $x \mapsto x^{*}$ is an anti-automorphism on $\mathcal{H}_{n}$ defined by $T_{i}^{*}=T_{i}$ for $i=1, \ldots, n-1$. Then it is known by [DJM, Theorem 3.26] that the set

$$
\left\{m_{\mathfrak{s t}} \mid \mathfrak{s}, \mathfrak{t} \in \operatorname{Std}(\lambda) \text { for some } \lambda \in \mathcal{P}_{n, r}\right\}
$$

gives a cellular basis of $\mathcal{H}$ with respect to the dominance order on $\mathcal{P}_{n, r}$ in the sense of [GL]. In particular, if we denote by $h \mapsto h^{*}$ the anti-automorphism on $\mathcal{H}$ defined by $T_{i}^{*}=T_{i}$ for $i=0, \ldots, n-1$, we have $m_{\mathfrak{s t}}^{*}=m_{\mathfrak{t} \mathfrak{s}}$.

1.3. Here we recall the concept of semistandard tableau in the case of multipartitions due to [DJM]. We consider the set $X$ of pairs $(i, k)$ with $1 \leq i \leq n, 1 \leq$ $k \leq r$, and define a total order on this set by $\left(i_{1}, k_{1}\right)<\left(i_{2}, k_{2}\right)$ if $k_{1}<k_{2}$, or if $k_{1}=k_{2}$ and $i_{1}<i_{2}$. For an $r$-partition $\lambda$ of $n$, a Tableau $T$ of shape $\lambda$ is defined as a Young diagram $\lambda$ with an element of $X$ attached to each node of $\lambda$. For each $(i, k) \in X$, let $\mu_{i}^{(k)}$ be the number of entries of $T$ containing $(i, k)$. Then $\mu=\left(\mu_{j}^{(k)}\right)$ is an $r$-composition of $n$. The Tableau $T$ is called a $\lambda$-tableau of type $\mu$. A Tableau $T=\left(T^{(1)}, \ldots, T^{(r)}\right)$ of shape $\lambda$ is called a semistandard tableau if it satisfies the properties; the entries of $T^{(i)}$ are weakly increasing along the rows, strictly increasing along the columns with respect to $X$, and furthermore the entries of $T^{(k)}$ consist of $\left(i, k^{\prime}\right)$ with $k^{\prime} \geq k$. We denote by $\mathcal{T}_{0}(\lambda, \mu)$ the set of semistandard tableau of shape $\lambda$ and type $\mu$ for $\lambda \in \mathcal{P}_{n, r}$ and $\mu \in \widetilde{\mathcal{P}}_{n, r}$. Note that $\mathcal{T}_{0}(\lambda, \mu)$ is empty unless $\lambda \unrhd \mu$. 
Let $\mathfrak{t}$ be a standard tableau of shape $\lambda$. For $\mu \in \widetilde{\mathcal{P}}_{n, r}$, we construct a tableau $\mu(\mathfrak{t})$ from $\mathfrak{t}$ as follows; replace the entry $j$ in $\mathfrak{t}$ by $(i, k)$ if $j$ appears in the $i$-th row of the $k$-th component $\left(\mathfrak{t}^{\mu}\right)^{(k)}$ of $\mathfrak{t}^{\mu} . \mu(\mathfrak{t})$ is a $\lambda$-tableau of type $\mu$, but it is not necessarily semistandard.

1.4. For each $\mu \in \widetilde{\mathcal{P}}_{n, r}$, we define a right $\mathcal{H}$-module $M^{\mu}$ by $M^{\mu}=m_{\mu} \mathcal{H}$. It is known by [DJM, Theorem 4.14] that $M^{\mu}$ is a free $R$-module with basis

$$
\left\{m_{S \mathfrak{t}} \mid S \in \mathcal{T}_{0}(\lambda, \mu), \mathfrak{t} \in \operatorname{Std}(\lambda) \text { for some } \lambda \in \mathcal{P}_{n, r}\right\},
$$

where

$$
m_{S \mathfrak{t}}=\sum_{\substack{\mathfrak{s} \in \operatorname{Std}(\lambda) \\ \mu(\mathfrak{s})=S}} q^{l(d(\mathfrak{s}))+l(d(\mathfrak{t}))} m_{\mathfrak{s t}} .
$$

A subset $\Lambda$ of $\widetilde{\mathcal{P}}_{n, r}(\mathbf{m})$ is called a saturated set if any partition $\lambda$ such that $\lambda \unrhd \mu$ for some $\mu \in \Lambda$ is contained in $\Lambda$. We denote by $\Lambda^{+}$the set of $r$-partitions of $n$ contained in $\Lambda$. Put $M=\bigoplus_{\mu \in \Lambda} M^{\mu}$. The cyclotomic $q$-Schur algebra $\mathcal{S}(\Lambda)$ associated to $\mathcal{H}$ (and to $\Lambda$ ) is defined by

$$
\mathcal{S}(\Lambda)=\operatorname{End}_{\mathcal{H}}(M)=\bigoplus_{\nu, \mu \in \Lambda} \operatorname{Hom}_{\mathcal{H}}\left(M^{\nu}, M^{\mu}\right) .
$$

We consider the structure of $\operatorname{Hom}_{\mathcal{H}}\left(M^{\nu}, M^{\mu}\right)$ for $\mu, \nu \in \Lambda$. Let $M^{\nu *}=\left(M^{\nu}\right)^{*}$ be the image of $M^{\nu}$ under $*$. We have $M^{\nu *}=\mathcal{H} m_{\nu}$. It is easy to see that for any $m \in M^{\nu *} \cap M^{\mu}=\mathcal{H} m_{\nu} \cap m_{\mu} \mathcal{H}$, the map $m_{\nu} h \mapsto m h(h \in \mathcal{H})$ gives rise to an $\mathcal{H}$-module homomorphism $\varphi_{m}: M^{\nu} \rightarrow M^{\mu}$. It is known by [DJM], Corollary 5.17] that the map $\varphi \mapsto \varphi\left(m_{\nu}\right)$ gives an isomorphism of $R$-modules

$$
\operatorname{Hom}_{\mathcal{H}}\left(M^{\nu}, M^{\mu}\right) \rightarrow M^{\nu *} \cap M^{\mu} .
$$

Suppose that $\mu, \nu \in \Lambda$, and $\lambda \in \Lambda^{+}$. We define for $S \in \mathcal{T}_{0}(\lambda, \mu), T \in \mathcal{T}_{0}(\lambda, \nu)$

$$
m_{S T}=\sum_{\substack{\mathfrak{s}, \mathfrak{t} \in \operatorname{Std}(\lambda) \\ \mu(\mathfrak{s})=S, \nu(\mathfrak{t})=T}} q^{l(d(\mathfrak{s}))+l(d(\mathfrak{t}))} m_{\mathfrak{s t}} .
$$

Then it is known by [DJM, Proposition 6.3] that the set

$$
\left\{m_{S T} \mid S \in \mathcal{T}_{0}(\lambda, \mu), T \in \mathcal{T}_{0}(\lambda, \nu) \text { for some } \lambda \in \Lambda^{+}\right\}
$$

gives rise to a basis of $M^{\nu *} \cap M^{\mu}$. We denote by $\varphi_{S T}$ the element of $\operatorname{Hom}_{\mathcal{H}}\left(M^{\nu}, M^{\mu}\right)$ corresponding to $m_{S T}$ via the isomorphism (1.4.3). Thus $\varphi_{S T}$ is a map $M^{\nu} \rightarrow M^{\mu}$ defined by $\varphi_{S T}\left(m_{\nu} h\right)=m_{S T} h$ for any $h \in \mathcal{H}$. For each $\lambda \in \Lambda^{+}$, put $\mathcal{T}_{0}(\lambda)=$ $\bigcup_{\mu \in \Lambda} \mathcal{T}_{0}(\lambda, \mu)$. The fundamental result of Dipper, James and Mathas is the following theorem.

Theorem $1.5([\overline{D J M})$. The cyclotomic $q$-Schur algebra $\mathcal{S}(\Lambda)$ is a cellular algebra with a cellular basis

$$
\mathcal{C}(\Lambda)=\left\{\varphi_{S T} \mid S, T \in \mathcal{T}_{0}(\lambda) \text { for some } \lambda \in \Lambda^{+}\right\} .
$$

1.6. For $\lambda \in \Lambda^{+}$, let $T^{\lambda}=\lambda\left(\mathfrak{t}^{\lambda}\right)$. Then $T^{\lambda}$ is a semistandard tableau obtained from $\mathfrak{t}^{\lambda}$ by replacing the entries $j$ in $\left(\mathfrak{t}^{\lambda}\right)^{(k)}$ by $(j, k)$. Then $\mathfrak{t}=\mathfrak{t}^{\lambda}$ is the unique standard tableau such that $\lambda(\mathfrak{t})=T^{\lambda}$. It follows that $m_{T^{\lambda} T^{\lambda}}=m_{\mathfrak{t}^{\lambda} \mathfrak{t}^{\lambda}}=m_{\lambda}$, and $\varphi_{T^{\lambda} T^{\lambda}}$ is the identity element in $\operatorname{Hom}_{\mathcal{H}}\left(M^{\lambda}, M^{\lambda}\right)$. We put $\varphi_{\lambda}=\varphi_{T^{\lambda} T^{\lambda}}$. 
For each $\lambda \in \Lambda^{+}$, we define $\mathcal{S}^{\vee \lambda}$ as the $R$-submodule of $\mathcal{S}(\Lambda)$ spanned by $\varphi_{S T}$, where $S, T \in \mathcal{T}_{0}\left(\lambda^{\prime}, \mu\right)$ for various $\lambda^{\prime} \in \Lambda^{+}$such that $\lambda^{\prime} \triangleright \lambda$, and for various $\mu \in \Lambda$. Then $\mathcal{S}^{\vee \lambda}$ is a two-sided ideal of $\mathcal{S}(\Lambda)$, and we define the Weyl module $W^{\lambda}$ as the right $\mathcal{S}(\Lambda)$-submodule of $\mathcal{S}(\Lambda) / \mathcal{S}^{\vee \lambda}$ generated by the image of $\varphi_{\lambda} \in \mathcal{S}(\Lambda)$. For each $T \in \mathcal{T}_{0}(\lambda)$, let $\varphi_{T}$ be the image of $\varphi_{T^{\lambda} T}$ in $W^{\lambda}$. Then the following holds: $W^{\lambda}$ is an $R$-free module with basis $\left\{\varphi_{T} \mid T \in \mathcal{T}_{0}(\lambda)\right\}$. There exists a canonical bilinear form $\langle$,$\rangle on W^{\lambda}$ determined by

$$
\varphi_{T^{\lambda} S} \varphi_{T T^{\lambda}} \equiv\left\langle\varphi_{S}, \varphi_{T}\right\rangle \varphi_{T^{\lambda} T^{\lambda}} \bmod \mathcal{S}^{\vee \lambda}
$$

Let $\operatorname{rad} W^{\lambda}=\left\{x \in W^{\lambda} \mid\langle x, y\rangle=0\right.$ for any $\left.y \in W^{\lambda}\right\}$. Then $\operatorname{rad} W^{\lambda}$ is an $\mathcal{S}(\Lambda)$ submodule of $W^{\lambda}$. Put $L^{\lambda}=W^{\lambda} / \operatorname{rad} W^{\lambda}$. Assume that $R$ is a field. Then it is known by DJM that $L^{\lambda}$ is a (non-zero) absolutely irreducible module, and that the set $\left\{L^{\lambda} \mid \lambda \in \Lambda^{+}\right\}$gives a complete set of non-isomorphic irreducible $\mathcal{S}(\Lambda)$-modules.

\section{Parabolic type subalgebras of $\mathcal{S}(\Lambda)$}

2.1. In Sa Sawada constructed a subalgebra $\mathcal{S}^{0}$ of $\mathcal{S}(\Lambda)$ and showed that its quotient algebra $\overline{\mathcal{S}}^{0}$ coincides with the cyclotomic $q$-Schur algebra associated to the modified Ariki-Koike algebra discussed in [SawS] under some condition on parameters (see Introduction). $\mathcal{S}^{0}$ is regarded, in some sense, as a Borel type subalgebra of $\mathcal{S}(\Lambda)$. In this section, we extend his result to a more general situation, i.e, to the parabolic type subalgebras.

2.2. Let $\Lambda$ and $\Lambda^{+}$be as in Section 1 . We fix $\mathbf{p}=\left(r_{1}, \ldots, r_{g}\right) \in \mathbb{Z}_{>0}^{g}$ such that $r=r_{1}+\cdots+r_{g}$ for some $g$, and put $p_{k}=\sum_{i=1}^{k-1} r_{i}$ for $k=1, \ldots, g$ with $p_{1}=0$. For each $\mu=\left(\mu^{(1)}, \ldots, \mu^{(r)}\right) \in \Lambda$, put

$$
\alpha_{\mathbf{p}}(\mu)=\left(n_{1}, \ldots, n_{g}\right), \quad \mathbf{a}_{\mathbf{p}}(\mu)=\left(a_{1}, \ldots, a_{g}\right),
$$

where $n_{k}=\sum_{i=1}^{r_{k}}\left|\mu^{\left(p_{k}+i\right)}\right|$ and $a_{k}=\sum_{i=1}^{k-1} n_{i}$ for $k=1, \ldots, g$ with $a_{1}=0$. By making use of $\mathbf{p}$, we express the $r$-compositions as the $g$-tuples of multi-compositions as follows: let $\mu=\left(\mu^{(1)}, \ldots, \mu^{(r)}\right) \in \widetilde{\mathcal{P}}_{n, r}$. We write $\mu$ as $\left(\mu^{[1]}, \ldots, \mu^{[g]}\right)$, where $\mu^{[k]}=\left(\mu^{\left(p_{k}+1\right)}, \ldots, \mu^{\left(p_{k}+r_{k}\right)}\right)$ is an $r_{k}$-composition of $n_{k}$. Note that $\mathbf{a}_{\mathbf{p}}(\mu)$ (resp. $\left.\alpha_{\mathbf{p}}(\mu)\right)$ coincides with $\mathbf{a}(\mu)$ (resp. $\left.\alpha(\mu)\right)$ in 1.2 in the special case where $\mathbf{p}=\left(1^{r}\right)$.

We define a partial order on $\mathbb{Z}_{\geq 0}^{g}$ by $\mathbf{a}=\left(a_{1}, \ldots, a_{g}\right) \geq \mathbf{b}=\left(b_{1}, \ldots, b_{g}\right)$ if $a_{k} \geq b_{k}$ for $k=1, \ldots, g$. We write $\mathbf{a}>\mathbf{b}$ if $\mathbf{a} \geq \mathbf{b}$ and $\mathbf{a} \neq \mathbf{b}$. The following properties are easily verified.

(2.2.1) Let $\mu, \nu \in \Lambda, \lambda \in \Lambda^{+}$. Then we have:

(i) $\mathbf{a}_{\mathbf{p}}(\mu)=\mathbf{a}_{\mathbf{p}}(\nu)$ if and only if $\alpha_{\mathbf{p}}(\mu)=\alpha_{\mathbf{p}}(\nu)$.

(ii) If $\nu \unrhd \mu$, then $\mathbf{a}_{\mathbf{p}}(\nu) \geq \mathbf{a}_{\mathbf{p}}(\mu)$. In particular, if $\mathcal{T}_{0}(\lambda, \mu) \neq \emptyset$, then $\lambda \unrhd \mu$ (cf. 1.3), and so $\mathbf{a}_{\mathbf{p}}(\lambda) \geq \mathbf{a}_{\mathbf{p}}(\mu)$.

For each $\lambda \in \Lambda^{+}, \mu \in \Lambda$, we define a set $\mathcal{T}_{0}^{\mathbf{p}}(\lambda, \mu)$ by $\mathcal{T}_{0}(\lambda, \mu)$ if $\mathbf{a}_{\mathbf{p}}(\lambda)=\mathbf{a}_{\mathbf{p}}(\mu)$ and by the empty set otherwise. Put $\mathcal{T}_{0}^{\mathbf{p}}(\lambda)=\bigcup_{\mu \in \Lambda} \mathcal{T}_{0}^{\mathbf{p}}(\lambda, \mu)$. 
Example 2.3. Let $n=20, r=5$ and take $\mu=\left(21 ; 121 ; 32 ; 1^{3} ; 41\right) \in \widetilde{\mathcal{P}}_{20,5}$. Let $\mathbf{p}=$ $(2,2,1)$. Then $\alpha_{\mathbf{p}}(\mu)=(7,8,5)$ and $\alpha_{\mathbf{p}}(\mu)=(0,7,15)$. We have $\mu=\left(\mu^{[1]}, \mu^{[2]}, \mu^{[3]}\right)$ with $\mu^{[1]}=(21 ; 121), \mu^{[2]}=\left(32 ; 1^{3}\right), \mu^{[3]}=(41)$.

2.4. Let $\mathcal{C}^{\mathbf{P}}=\mathcal{C}^{\mathbf{p}}(\Lambda)$ be the set of $\varphi_{S T} \in \mathcal{C}(\Lambda)$ for $S \in \mathcal{T}_{0}(\lambda, \mu), T \in \mathcal{T}_{0}(\lambda, \nu)$, where $\mu, \nu \in \Lambda, \lambda \in \Lambda^{+}$are taken subject to the condition that $\mathbf{a}_{\mathbf{p}}(\lambda)>\mathbf{a}_{\mathbf{p}}(\mu)$ if $\alpha_{\mathbf{p}}(\mu) \neq \alpha_{\mathbf{p}}(\nu)$. We define an $R$-submodule $\mathcal{S}^{\mathbf{p}}=\mathcal{S}^{\mathbf{p}}(\Lambda)$ of $\mathcal{S}(\Lambda)$ as the $R$-span of $\mathcal{C}^{\mathbf{p}}$. We will see that $\mathcal{S}^{\mathbf{p}}$ is a subalgebra of $\mathcal{S}(\Lambda)$ and that $\mathcal{S}^{\mathbf{p}}$ turns out to be a standardly based algebra in the sense of Du and Rui [DR]. Note that in the case where $\mathbf{p}=\left(1^{r}\right), \mathcal{S}^{\mathbf{p}}$ coincides with $\mathcal{S}^{0}$.

First we note that the identity element $1_{\mathcal{S}(\Lambda)}$ is contained in $\mathcal{S}^{\mathbf{p}}$. In fact, one can write $1_{\mathcal{S}(\Lambda)}=\sum_{\mu \in \Lambda} \varphi_{\mu}$, where $\varphi_{\mu} \in \mathcal{S}(\Lambda)$ is the identity map on $M^{\mu}$. Since $\varphi_{\mu}$ is written as a linear combination of $\varphi_{S T}$ with $S, T \in \mathcal{T}_{0}(\lambda, \mu)$, we see that $1_{\mathcal{S}(\Lambda)} \in \mathcal{S}^{\mathbf{p}}$.

In order to relate $\mathcal{S}^{\mathbf{p}}$ to the standardly based algebra, we introduce a different kind of labeling for $\mathcal{C}^{\mathbf{P}}$, following the idea of [Sa. Let us define a subset $\Sigma^{\mathbf{p}}$ of $\Lambda^{+} \times\{0,1\}$ by

$$
\begin{aligned}
\Sigma^{\mathbf{p}}=\left(\Lambda^{+} \times\{0,1\}\right) \backslash\left\{(\lambda, 1) \mid \mathcal{T}_{0}(\lambda, \mu)=\emptyset\right. \\
\text { for any } \left.\mu \in \Lambda \text { such that } \mathbf{a}_{\mathbf{p}}(\lambda)>\mathbf{a}_{\mathbf{p}}(\mu)\right\} .
\end{aligned}
$$

We define a partial order $\geq$ on $\Sigma^{\mathbf{p}}$ by $\left(\lambda_{1}, \varepsilon_{1}\right)>\left(\lambda_{2}, \varepsilon_{2}\right)$ if $\lambda_{1} \triangleright \lambda_{2}$ or $\lambda_{1}=\lambda_{2}$ and $\varepsilon_{1}>\varepsilon_{2}$.

For each $\eta=(\lambda, \varepsilon) \in \Sigma^{\mathbf{p}}$, put

$$
\begin{aligned}
& I^{\mathbf{p}}(\eta)= \begin{cases}\mathcal{T}_{0}^{\mathbf{p}}(\lambda) & \text { if } \varepsilon=0, \\
\bigcup_{\substack{\mu \in \Lambda \\
\mathbf{a}_{\mathbf{p}}(\lambda)>\mathbf{a}_{\mathbf{p}}(\mu)}} \mathcal{T}_{0}(\lambda, \mu) & \text { if } \varepsilon=1,\end{cases} \\
& J^{\mathbf{p}}(\eta)= \begin{cases}\mathcal{T}_{0}^{\mathbf{p}}(\lambda) & \text { if } \varepsilon=0, \\
\mathcal{T}_{0}(\lambda) & \text { if } \varepsilon=1 .\end{cases}
\end{aligned}
$$

Note that $I^{\mathbf{p}}(\eta)$ and $J^{\mathbf{p}}(\eta)$ are not empty. For $\eta \in \Sigma^{\mathbf{p}}$, if we put,

$$
\mathcal{C}^{\mathbf{p}}(\eta)=\left\{\varphi_{S T} \mid S \in I^{\mathbf{p}}(\eta), T \in J^{\mathbf{p}}(\eta)\right\}
$$

we see easily that

$$
\mathcal{C}^{\mathbf{p}}=\coprod_{\eta \in \Sigma^{\mathbf{p}}} \mathcal{C}^{\mathbf{p}}(\eta)
$$

For each $\eta \in \Sigma^{\mathbf{p}}$, we define a submodule $\left(\mathcal{S}^{\mathbf{p}}\right)^{\vee \eta}$ of $\mathcal{S}^{\mathbf{p}}$ as the $R$-span of $\varphi_{S T}$, where $S \in I^{\mathbf{p}}\left(\eta^{\prime}\right), T \in J^{\mathbf{p}}\left(\eta^{\prime}\right)$ for some $\eta^{\prime} \in \Sigma^{\mathbf{p}}$ such that $\eta^{\prime}>\eta$.

By using the cellular structure of $\mathcal{S}(\Lambda)$, the following result can be proved in a similar way as in [Sa, Lemma 2.4]. 
Lemma 2.5. Take $\lambda_{i} \in \Lambda^{+}, \mu_{i}, \nu_{i} \in \Lambda$ for $i=1,2$ such that $\nu_{1}=\mu_{2}$. Then for $\varphi_{S_{i} T_{i}} \in \mathcal{C}^{\mathbf{p}}$ with $S_{i} \in \mathcal{T}_{0}\left(\lambda_{i}, \mu_{i}\right), T_{i} \in \mathcal{T}_{0}\left(\lambda_{i}, \nu_{i}\right)$, the following hold:

$$
\begin{aligned}
& \varphi_{S_{1} T_{1}} \cdot \varphi_{S_{2} T_{2}}= \\
& = \begin{cases}\sum_{\varphi_{S T} \in \mathcal{C}^{\mathbf{p}}\left(\lambda_{1}, 0\right)} r_{S T} \varphi_{S T}+\sum_{\lambda \triangleright \lambda_{1}} \sum_{\varphi_{S T} \in \mathcal{C}^{\mathbf{p}}(\lambda)} r_{S T} \varphi_{S T} & \text { if } \varphi_{S_{1} T_{1}} \in \mathcal{C}^{\mathbf{p}}\left(\lambda_{1}, 0\right), \\
\sum_{\lambda \unrhd \lambda_{1}} \sum_{\varphi_{S T} \in \mathcal{C}^{\mathbf{p}}(\lambda, 1)} r_{S T} \varphi_{S T} & \text { if } \varphi_{S_{1} T_{1}} \in \mathcal{C}^{\mathbf{p}}\left(\lambda_{1}, 1\right), \\
\sum_{\lambda \unrhd \lambda_{2}} \sum_{\varphi_{S T} \in \mathcal{C}^{\mathbf{p}}(\lambda)} r_{S T} \varphi_{S T} & \text { if } \varphi_{S_{2} T_{2}} \in \mathcal{C}^{\mathbf{p}}\left(\lambda_{2}, 0\right), \\
\sum_{\lambda \unrhd \lambda_{2}} \sum_{\varphi_{S T} \in \mathcal{C}^{\mathbf{p}}(\lambda, 1)} r_{S T} \varphi_{S T} & \text { if } \varphi_{S_{2} T_{2}} \in \mathcal{C}^{\mathbf{p}}\left(\lambda_{2}, 1\right),\end{cases}
\end{aligned}
$$

where $r_{S T} \in R$ and $\mathcal{C}^{\mathbf{P}}(\lambda)=\mathcal{C}^{\mathbf{p}}(\lambda, 0) \cup \mathcal{C}^{\mathbf{p}}(\lambda, 1)$.

The following theorem is a generalization of [Sa, Theorem 2.6]. The proof is done similarly by using Lemma 2.5 .

Theorem 2.6. $\mathcal{S}^{\mathbf{p}}$ is a subalgebra of $\mathcal{S}(\Lambda)$ containing the identity element of $\mathcal{S}(\Lambda)$. Moreover, $\mathcal{S}^{\mathbf{p}}$ turns out to be a standardly based algebra with the standard basis $\mathcal{C}^{\mathbf{p}}$ in the sense of $\left[\mathrm{DR}\right.$, i.e., the following holds: for any $\varphi \in \mathcal{S}^{\mathbf{p}}, \varphi_{S T} \in \mathcal{C}^{\mathbf{p}}(\eta)$, we have

$$
\begin{aligned}
\varphi \cdot \varphi_{S T} & \equiv \sum_{S^{\prime} \in I^{\mathbf{P}}(\eta)} f_{S^{\prime}} \varphi_{S^{\prime} T} & \bmod \left(\mathcal{S}^{\mathbf{p}}\right)^{\vee \eta}, \\
\varphi_{S T} \cdot \varphi & \equiv \sum_{T^{\prime} \in J^{\mathbf{p}}(\eta)} f_{T^{\prime}}^{\prime} \varphi_{S T^{\prime}} & \bmod \left(\mathcal{S}^{\mathbf{p}}\right)^{\vee \eta}
\end{aligned}
$$

with $f_{S^{\prime}}, f_{T^{\prime}}^{\prime} \in R$, where in the first formula $f_{S^{\prime}}$ depends on $\left(\varphi, S, S^{\prime}\right)$ but does not depend on $T$, and in the second formula $f_{T^{\prime}}^{\prime}$ depends on $\left(\varphi, T, T^{\prime}\right)$ but does not depend on $S$.

2.7. For each $\eta \in \Sigma^{\mathbf{p}}$, let $\diamond Z_{\mathbf{p}}^{\eta}$ be an $R$-module with a basis $\left\{\varphi_{S}^{\eta} \mid S \in I^{\mathbf{p}}(\eta)\right\}$, and let $Z_{\mathbf{p}}^{\eta}$ be an $R$-module with a basis $\left\{\varphi_{T}^{\eta} \mid T \in J^{\mathbf{p}}(\eta)\right\}$. In view of Theorem 2.6 , one can define actions of $\mathcal{S}^{\mathbf{p}}$ on $\diamond Z_{\mathbf{p}}^{\eta}$ and on $Z_{\mathbf{p}}^{\eta}$ by

$$
\begin{aligned}
\varphi \cdot \varphi_{S}^{\eta} & =\sum_{S^{\prime} \in I^{\mathbf{p}}(\eta)} f_{S^{\prime}} \varphi_{S^{\prime}}^{\eta} \quad\left(S \in I^{\mathbf{p}}(\eta), \varphi \in \mathcal{S}^{\mathbf{p}}\right), \\
\varphi_{T}^{\eta} \cdot \varphi & =\sum_{T^{\prime} \in J^{\mathbf{p}}(\eta)} f_{T^{\prime}}^{\prime} \varphi_{T^{\prime}}^{\eta}
\end{aligned}
$$

where $f_{S^{\prime}}, f_{T^{\prime}}^{\prime}$ are as in the theorem. Then $\diamond Z_{\mathbf{p}}^{\eta}\left(\right.$ resp. $Z_{\mathbf{p}}^{\eta}$ ) has a structure of the left $\mathcal{S}^{\mathbf{p}}$-module (resp. the right $\mathcal{S}^{\mathbf{p}}$-module). Moreover, the theorem implies, for any $\varphi_{U T}, \varphi_{S V} \in \mathcal{C}^{\mathbf{p}}(\eta)$, that there exists $f_{T S} \in R$ (independent of the choice of $U, V)$ such that

$$
\varphi_{U T} \varphi_{S V} \equiv f_{T S} \varphi_{U V} \quad \bmod \left(\mathcal{S}^{\mathbf{p}}\right)^{\vee \eta} .
$$


We define a bilinear form $\beta_{\eta}: \diamond_{\mathbf{p}}^{\eta} \times Z_{\mathbf{p}}^{\eta} \rightarrow R$ by $\beta_{\eta}\left(\varphi_{S}^{\eta}, \varphi_{T}^{\eta}\right)=f_{T S}$ for $S \in$ $I^{\mathbf{p}}(\eta), T \in J^{\mathbf{p}}(\eta)$. Put

$$
\operatorname{rad} Z_{\mathbf{p}}^{\eta}=\left\{y \in Z_{\mathbf{p}}^{\eta} \mid \beta_{\eta}(x, y)=0 \text { for any } x \in{ }^{\diamond} Z_{\mathbf{p}}^{\eta}\right\} .
$$

Then $\operatorname{rad} Z_{\mathbf{p}}^{\eta}$ is an $\mathcal{S}^{\mathbf{p}}$-submodule of $Z_{\mathbf{p}}^{\eta}$ and we define the quotient module $L_{\mathbf{p}}^{\eta}=$ $Z_{\mathbf{p}}^{\eta} / \operatorname{rad} Z_{\mathbf{p}}^{\eta}$. By the general theory of standardly based algebras (see [DR]), we obtain the following corollary, which is a strengthened form of Sa, Proposition 3.7].

Corollary 2.8. Assume that $R$ is a field. Then:

(i) $L_{\mathbf{p}}^{\eta}$ is an absolutely irreducible $\mathcal{S}^{\mathbf{p}}$-module if it is non-zero.

(ii) The set $\left\{L_{\mathbf{p}}^{\eta} \neq 0 \mid \eta \in \Sigma^{\mathbf{p}}\right\}$ gives a complete set of non-isomorphic irreducible right $\mathcal{S}^{\mathbf{p}}$-modules.

Remarks 2.9. (i) In $\mathrm{Sa}$, only the case $Z_{\mathbf{p}}^{(\lambda, 0)}$ is discussed (for $\mathbf{p}=\left(1^{r}\right)$ ). In that case (for arbitrary $\mathbf{p}$ ), we have the following description on the basis of $Z_{\mathbf{p}}^{(\lambda, 0)}$ as in the case of the Weyl module $W^{\lambda}$. For each $\lambda \in \Lambda^{+}, \varphi_{\lambda}=\varphi_{T^{\lambda} T^{\lambda}}$ is contained in $\mathcal{C}^{\mathbf{p}}(\lambda, 0)$. We consider the $\mathcal{S}^{\mathbf{p}}$-submodule $W_{\mathbf{p}}^{\lambda}$ of $\mathcal{S}^{\mathbf{p}} /\left(\mathcal{S}^{\mathbf{p}}\right)^{\vee(\lambda, 0)}$ generated by the image of $\varphi_{\lambda}$. Since $T^{\lambda} \in I^{\mathbf{p}}(\lambda, 0)$, we see that $\varphi_{T^{\lambda} T} \in \mathcal{C}^{\mathbf{p}}(\lambda, 0)$ for any $T \in J^{\mathbf{p}}(\lambda, 0)$. We denote by $\varphi_{T}^{\prime}$ the image of $\varphi_{T^{\lambda} T}$ on $\mathcal{S}^{\mathbf{p}} /\left(\mathcal{S}^{\mathbf{p}}\right)^{\vee(\lambda, 0)}$. Then one can check that $\varphi_{T}^{\prime} \in W_{\mathbf{p}}^{\lambda}$ and that the map $\varphi_{T} \rightarrow \varphi_{T}^{\prime}$ gives an isomorphism $Z_{\mathbf{p}}^{(\lambda, 0)} \rightarrow W_{\mathbf{p}}^{\lambda}$ of $\mathcal{S}^{\mathbf{p}_{-}}$ modules. In particular, we see that $Z_{\mathbf{p}}^{(\lambda, 0)}$ is generated by $\varphi_{T^{\lambda}}^{(\lambda, 0)}$ as an $\mathcal{S}^{\mathbf{p}}$-module.

However, the above argument cannot be applied to $Z_{\mathbf{p}}^{(\lambda, 1)}$ since $\varphi_{T^{\lambda} T} \notin \mathcal{S}^{\mathbf{p}}$ for $T \in J^{\mathbf{p}}(\lambda, 1) \backslash J^{\mathbf{p}}(\lambda, 0)$. It is not known whether $Z_{\mathbf{p}}^{(\lambda, 1)}$ is generated by one element as an $\mathcal{S}^{\mathbf{p}}$-module.

(ii) For any $\lambda \in \Lambda^{+}$, we have $L_{\mathbf{p}}^{(\lambda, 0)} \neq 0$. In fact, since $T^{\lambda} \in I^{\mathbf{P}}(\lambda, 0) \cap J^{\mathbf{p}}(\lambda, 0)$, we have $f_{T^{\lambda} T^{\lambda}}=1$. This implies that $\beta_{(\lambda, 0)}\left(\varphi_{T^{\lambda}}^{(\lambda, 0)}, \varphi_{T^{\lambda}}^{(\lambda, 0)}\right)=1$ and we see that $\operatorname{rad} Z_{\mathbf{p}}^{(\lambda, 0)} \neq Z_{\mathbf{p}}^{(\lambda, 0)}$.

This argument cannot be applied to $Z_{\mathbf{p}}^{(\lambda, 1)}$ since $T^{\lambda} \notin I^{\mathbf{P}}(\lambda, 1)$ and so $\varphi_{T^{\lambda}}^{(\lambda, 1)} \notin$ $\diamond Z_{\mathbf{p}}^{(\lambda, 1)}$. It is not known when $L_{\mathbf{p}}^{(\lambda, 1)} \neq 0$.

2.10. Recall that $\varphi \mapsto \varphi^{*}$ is the anti-automorphism on $\mathcal{S}(\Lambda)$ defined by $\varphi_{S T} \mapsto$ $\varphi_{T S}$, related to the cellular structure. Let $\mathcal{S}^{\mathbf{p} *}=\left(\mathcal{S}^{\mathbf{p}}\right)^{*}$ be the image of $\mathcal{S}^{\mathbf{p}}$ under the map $*$. Then $\mathcal{S}^{\mathbf{p} *}$ is a subalgebra of $\mathcal{S}(\Lambda)$, and it is easy to check that $\mathcal{S}^{\mathbf{p} *}$ is a standardly based algebra with the standard basis $\mathcal{C}^{\mathbf{p} *}=\coprod_{\eta \in \Sigma^{\mathbf{p}}} \mathcal{C}^{\mathbf{p}}(\eta)^{*}$, where

$$
\mathcal{C}^{\mathbf{p}}(\eta)^{*}=\left\{\varphi_{S T} \in \mathcal{C}(\Lambda) \mid S \in J^{\mathbf{p}}(\eta), T \in I^{\mathbf{p}}(\eta)\right\} .
$$

In a similar way as in $\mathrm{Sa}$, Proposition 3.2], one can show the following result.

Proposition 2.11. We have $\mathcal{S}(\Lambda)=\mathcal{S}^{\mathbf{p}} \cdot \mathcal{S}^{\mathbf{p} *}$.

2.12. Let $\widehat{\mathcal{S}}^{\mathbf{p}}$ be the $R$-submodule of $\mathcal{S}^{\mathbf{p}}$ spanned by

$$
\widehat{\mathcal{C}}^{\mathbf{p}}=\mathcal{C}^{\mathbf{p}} \backslash\left\{\varphi_{S T} \mid S, T \in \mathcal{T}_{0}^{\mathbf{p}}(\lambda) \text { for some } \lambda \in \Lambda^{+}\right\} .
$$

Then by the second and the fourth formulas in Lemma $2.5, \widehat{\mathcal{S}}^{\text {p }}$ turns out to be a two-sided ideal of $\mathcal{S}^{\mathbf{p}}$. We denote by $\overline{\mathcal{S}}^{\mathbf{p}}=\overline{\mathcal{S}}^{\mathbf{p}}(\Lambda)$ the quotient algebra $\mathcal{S}^{\mathbf{p}} / \widehat{\mathcal{S}}^{\mathbf{p}}$. Let 
$\pi: \mathcal{S}^{\mathbf{p}} \rightarrow \overline{\mathcal{S}}^{\mathbf{p}}$ be the natural projection, and put $\bar{\varphi}=\pi(\varphi)$ for $\varphi \in \mathcal{S}^{\mathbf{p}}$. It is easy to see that $\overline{\mathcal{S}}^{\mathrm{p}}$ is an $R$-free module with the basis

$$
\overline{\mathcal{C}}^{\mathbf{p}}=\left\{\bar{\varphi}_{S T} \mid S, T \in \mathcal{T}_{0}^{\mathbf{p}}(\lambda) \text { for } \lambda \in \Lambda^{+}\right\} .
$$

Similarly, one can define a quotient algebra $\overline{\mathcal{S}}^{\mathbf{p} *}=\mathcal{S}^{\mathbf{p} *} / \widehat{\mathcal{S}}^{\mathbf{p}}$, where $\widehat{\mathcal{S}}^{\mathbf{p} *}=\left(\widehat{\mathcal{S}}^{\mathbf{p}}\right)^{*}$ is a two-sided ideal of $\mathcal{S}^{\mathbf{p} *}$. Let $\pi^{\prime}$ be the natural projection $\mathcal{S}^{\mathbf{p} *} \rightarrow \overline{\mathcal{S}}^{\mathbf{p} *}$, and put $\bar{\varphi}^{\prime}=$ $\pi^{\prime}(\varphi)$ for $\varphi \in \mathcal{S}^{\mathbf{p} *}$. Then $\overline{\mathcal{S}}^{\mathbf{p} *}$ has an $R$-free basis $\overline{\mathcal{C}}^{\mathbf{p} *}=\left\{\bar{\varphi}_{S T}^{\prime} \mid S, T \in \mathcal{T}_{0}^{\mathbf{p}}(\lambda), \lambda \in\right.$ $\left.\Lambda^{+}\right\}$. It is clear that $\bar{\varphi}_{S T} \mapsto \bar{\varphi}_{S T}^{\prime}$ gives an isomorphism $\overline{\mathcal{S}}^{\mathbf{p}} \rightarrow \overline{\mathcal{S}}^{\mathrm{p} *}$ of $R$-algebras. On the other hand, the anti-algebra isomorphism $\mathcal{S}^{\mathbf{p}} \rightarrow \mathcal{S}^{\mathbf{p} *}$ induces an anti-algebra isomorphism $\overline{\mathcal{S}}^{\mathbf{p}} \rightarrow \overline{\mathcal{S}}^{\mathbf{p} *}, \bar{\varphi}_{S T} \mapsto \bar{\varphi}_{T S}^{\prime}$. It follows that the map $\bar{\varphi}_{S T} \mapsto \bar{\varphi}_{T S}$ induces an anti-algebra automorphism $*$ on $\overline{\mathcal{S}}^{\mathbf{p}}$. Thus we have the following theorem (cf. [Sa, Theorem 4.8]). Note that the second assertion is obtained from the cellular structure of $\mathcal{S}(\Lambda)$.

Theorem 2.13. $\overline{\mathcal{S}}^{\mathbf{p}}$ is a cellular algebra with a cellular basis $\overline{\mathcal{C}}^{\mathbf{p}}$, i.e., the following properties hold:

(i) $\bar{\varphi}_{S T} \mapsto\left(\bar{\varphi}_{S T}\right)^{*}=\bar{\varphi}_{T S}$ gives an anti-algebra automorphism $*$ on $\overline{\mathcal{S}}^{\mathbf{p}}$.

(ii) Let $\left(\overline{\mathcal{S}}^{\mathbf{p}}\right)^{\vee \lambda}$ be the $R$-submodule of $\overline{\mathcal{S}}^{\mathbf{p}}$ spanned by $\bar{\varphi}_{S T}$ such that $S, T \in$ $\mathcal{T}_{0}^{\mathbf{p}}\left(\lambda^{\prime}\right)$ with $\lambda^{\prime} \triangleright \lambda$. Then for any $\lambda \in \Lambda^{+}, S, T \in \mathcal{T}_{0}^{\mathbf{p}}(\lambda), \bar{\varphi} \in \overline{\mathcal{S}}^{\mathbf{p}}$,

$$
\bar{\varphi}_{S T} \cdot \bar{\varphi} \equiv \sum_{T^{\prime} \in \mathcal{T}_{0}^{\mathbf{p}}(\lambda)} r_{T^{\prime}} \bar{\varphi}_{S T^{\prime}} \quad \bmod \left(\overline{\mathcal{S}}^{\mathbf{p}}\right)^{\vee \lambda}
$$

where $r_{T^{\prime}} \in R$ depends on $\lambda, T, \bar{\varphi}$, but does not depend on $S$.

2.14. We apply the general theory of cellular algebras to $\overline{\mathcal{S}}^{\mathbf{p}}$. For each $\lambda \in \Lambda^{+}$, $\left(\overline{\mathcal{S}}^{\mathbf{p}}\right)^{\vee \lambda}$ is a two-sided ideal of $\overline{\mathcal{S}}^{\mathbf{p}}$, and we define the Weyl module $\bar{Z}_{\mathbf{p}}^{\lambda}$ as the $\overline{\mathcal{S}}^{\mathbf{p}}$ submodule of the right $\overline{\mathcal{S}}^{\mathbf{p}}$-module $\overline{\mathcal{S}}^{\mathbf{p}} /\left(\overline{\mathcal{S}}^{\mathbf{p}}\right)^{\vee \lambda}$ generated by $\bar{\varphi}_{T^{\lambda} T^{\lambda}}+\left(\overline{\mathcal{S}}^{\mathbf{p}}\right)^{\vee \lambda}$. Let $\bar{\varphi}_{T}$ be the image of $\bar{\varphi}_{T^{\lambda} T}$ on $\overline{\mathcal{S}}^{\mathbf{p}} /\left(\overline{\mathcal{S}}^{\mathbf{p}}\right)^{\vee \lambda}$. Then the set $\left\{\bar{\varphi}_{T} \mid T \in \mathcal{T}_{0}^{\mathbf{p}}(\lambda)\right\}$ gives a basis of $\bar{Z}_{\mathbf{p}}^{\lambda}$. The symmetric bilinear form $\langle,\rangle_{\mathbf{p}}: \bar{Z}_{\mathbf{p}}^{\lambda} \times \bar{Z}_{\mathbf{p}}^{\lambda} \rightarrow R$ is defined by the equation

$$
\left\langle\bar{\varphi}_{S}, \bar{\varphi}_{T}\right\rangle_{\mathbf{p}} \bar{\varphi}_{T^{\lambda} T^{\lambda}} \equiv \bar{\varphi}_{T^{\lambda} S} \bar{\varphi}_{T^{\lambda} \lambda} \quad \bmod \left(\overline{\mathcal{S}}^{\mathbf{p}}\right)^{\vee \lambda} .
$$

Then the radical $\operatorname{rad} \bar{Z}_{\mathbf{p}}^{\lambda}$ of $\bar{Z}_{\mathbf{p}}^{\lambda}$ with respect to this form is an $\overline{\mathcal{S}}^{\mathbf{p}}$-submodule of $\bar{Z}_{\mathbf{p}}^{\lambda}$, and we define an $\overline{\mathcal{S}}^{\mathbf{p}}$-module $\bar{L}_{\mathbf{p}}^{\lambda}$ by $\bar{L}_{\mathbf{p}}^{\lambda}=\bar{Z}_{\mathbf{p}}^{\lambda} / \operatorname{rad} \bar{Z}_{\mathbf{p}}^{\lambda}$. Since $\left\langle\bar{\varphi}_{T^{\lambda}}, \bar{\varphi}_{T^{\lambda}}\right\rangle_{\mathbf{p}}=1$, we see that $\bar{L}_{\mathbf{p}}^{\lambda} \neq 0$ for any $\lambda \in \Lambda^{+}$. By the general theory of cellular algebras, we have

Corollary 2.15. Suppose that $R$ is a field. Then, for any $\lambda \in \Lambda^{+}, \bar{L}_{\mathbf{p}}^{\lambda}$ is an absolutely irreducible $\overline{\mathcal{S}}^{\mathbf{p}}$-module, and the set $\left\{\bar{L}_{\mathbf{p}}^{\lambda} \mid \lambda \in \Lambda^{+}\right\}$gives a complete set of non-isomorphic irreducible $\overline{\mathcal{S}}^{\mathbf{p}}$-modules.

\section{Decomposition numbers for $\mathcal{S}(\Lambda), \mathcal{S}^{\mathbf{p}}$ and $\overline{\mathcal{S}}^{\mathbf{p}}$}

3.1. By the discussion in the previous section, we have the following diagram:

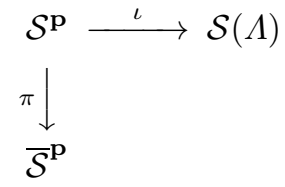


where $\iota$ is the inclusion map, and $\pi$ is the natural surjective map. We have constructed the Weyl modules $W^{\lambda}, Z_{\mathbf{p}}^{\eta}$ and $\bar{Z}_{\mathbf{p}}^{\lambda}$ for $\mathcal{S}(\Lambda), \mathcal{S}^{\mathbf{p}}$ and $\overline{\mathcal{S}}^{\mathbf{p}}$, and assuming that $R$ is a field, the irreducible modules $L^{\mu}, L_{\mathbf{p}}^{\eta^{\prime}}, \bar{L}_{\mathbf{p}}^{\mu}$, respectively for $\lambda, \mu \in \Lambda^{+}, \eta, \eta^{\prime} \in \Sigma^{\mathbf{p}}$. We consider the decomposition numbers

$$
\left[W^{\lambda}: L^{\mu}\right]_{\mathcal{S}(\Lambda)}, \quad\left[Z_{\mathbf{p}}^{\eta}: L_{\mathbf{p}}^{\eta^{\prime}}\right]_{\mathcal{S}^{\mathbf{p}}}, \quad\left[\bar{Z}_{\mathbf{p}}^{\lambda}: \bar{L}_{\mathbf{p}}^{\mu}\right]_{\mathcal{S}^{\mathbf{p}}}
$$

for $\mathcal{S}(\Lambda), \mathcal{S}^{\mathbf{p}}$ and $\overline{\mathcal{S}}^{\mathbf{p}}$. By using the above maps, we shall discuss the relationship among these decomposition numbers.

First we consider the relation between $\mathcal{S}^{\mathbf{p}}$ and $\overline{\mathcal{S}}^{\mathbf{p}}$. We regard an $\overline{\mathcal{S}}^{\mathbf{p}}$-module as an $\mathcal{S}^{\mathbf{p}}$-module through the map $\pi$. The following lemma is easily verified if we notice that $\pi\left(\left(\mathcal{S}^{\mathbf{p}}\right)^{\vee(\lambda, 0)}\right)=\overline{\mathcal{S}}^{\vee \lambda}$ and that $\beta_{(\lambda, 0)}\left(\varphi_{S}^{(\lambda, 0)}, \varphi_{T}^{(\lambda, 0)}\right)=\left\langle\bar{\varphi}_{S}, \bar{\varphi}_{T}\right\rangle_{\mathbf{p}}$ for $S, T \in \mathcal{T}_{0}^{\mathbf{p}}(\lambda)$.

Lemma 3.2. (i) For any $\lambda \in \Lambda^{+}$, the map $\varphi_{T}^{(\lambda, 0)} \mapsto \bar{\varphi}_{T}\left(T \in \mathcal{T}_{0}^{\mathbf{p}}(\lambda)\right)$ gives an isomorphism $Z_{\mathbf{p}}^{(\lambda, 0)} \stackrel{\sim}{\rightarrow} \bar{Z}_{\mathbf{p}}^{\lambda}$ of $\mathcal{S}^{\mathbf{p}}$-modules.

(ii) Assume that $R$ is a field. Then the above map induces an isomorphism $L_{\mathbf{p}}^{(\lambda, 0)} \stackrel{\sim}{\rightarrow} \bar{L}_{\mathbf{p}}^{\lambda}$ of $\mathcal{S}^{\mathbf{p}}$-modules.

The following proposition is proved in a similar way as in [Sa, Theorem 4.15] by taking the lemma into account.

Proposition 3.3. Assume that $R$ is a field. Then:

(i) The composition factors of $Z_{\mathbf{p}}^{(\lambda, 0)}$ are isomorphic to $L_{\mathbf{p}}^{(\mu, 0)}$ for some $\mu \in \Lambda^{+}$ such that $\lambda \triangleright \mu$.

(ii) For any $\lambda, \mu \in \Lambda^{+}$, we have $\left[\bar{Z}_{\mathbf{p}}^{\lambda}: \bar{L}_{\mathbf{p}}^{\mu}\right]_{\mathcal{S}^{\mathbf{p}}}=\left[Z_{\mathbf{p}}^{(\lambda, 0)}: L_{\mathbf{p}}^{(\mu, 0)}\right]_{\mathcal{S}^{\mathbf{p}}}$.

(iii) For $\lambda, \mu \in \Lambda^{+}$such that $\alpha_{\mathbf{p}}(\lambda) \neq \alpha_{\mathbf{p}}(\mu)$, we have $\left[\bar{Z}_{\mathbf{p}}^{\lambda}: \bar{L}_{\mathbf{p}}^{\mu}\right]_{\mathcal{S}^{\mathbf{p}}}=0$.

3.4. Next we consider the relation between $\mathcal{S}(\Lambda)$ and $\mathcal{S}^{\mathbf{p}}$. Since $\mathcal{S}^{\mathbf{p}}$ is a subalgebra of $\mathcal{S}(\Lambda)$, we regard an $\mathcal{S}(\Lambda)$-module as an $\mathcal{S}^{\mathbf{p}}$-module by restriction. Recall that $J^{\mathbf{p}}(\lambda, 0)=\mathcal{T}_{0}^{\mathbf{p}}(\lambda), J^{\mathbf{p}}(\lambda, 1)=\mathcal{T}_{0}(\lambda)$ for $\lambda \in \Lambda^{+}$. Thus the basis of $Z_{\mathbf{p}}^{(\lambda, 0)}$ is $\left\{\varphi_{T}^{(\lambda, 0)} \mid T \in \mathcal{T}_{0}^{\mathbf{P}}(\lambda)\right\}$, the basis of $Z_{\mathbf{p}}^{(\lambda, 1)}$ is $\left\{\varphi_{T}^{(\lambda, 1)} \mid T \in \mathcal{T}_{0}(\lambda)\right\}$, and the basis of $W^{\lambda}$ is $\left\{\varphi_{T} \mid T \in \mathcal{T}_{0}(\lambda)\right\}$, respectively. The following result is implicit in [Sa].

Lemma 3.5. For each $\lambda \in \Lambda^{+}$, the following hold:

(i) The map $\varphi_{T}^{(\lambda, 0)} \mapsto \varphi_{T}^{(\lambda, 1)}\left(T \in \mathcal{T}_{0}^{\mathbf{p}}(\lambda)\right)$ gives an injective homomorphism $Z_{\mathbf{p}}^{(\lambda, 0)} \rightarrow Z_{\mathbf{p}}^{(\lambda, 1)}$ of $\mathcal{S}^{\mathbf{p}}$-modules.

(ii) The $\operatorname{map} \varphi_{T}^{(\lambda, 1)} \mapsto \varphi_{T}\left(T \in \mathcal{T}_{0}(\lambda)\right)$ gives an isomorphism $Z_{\mathbf{p}}^{(\lambda, 1)} \stackrel{\sim}{\rightarrow} W^{\lambda}$ of $\mathcal{S}^{\mathbf{p}}$-modules.

Proof. Take $\varphi_{S T} \in \mathcal{C}(\Lambda)\left(S, T \in \mathcal{T}_{0}(\lambda)\right)$, and $\varphi \in \mathcal{S}^{\mathbf{p}}$. By the property of the cellular algebra $\mathcal{S}(\Lambda)$, we have

$$
\varphi_{S T} \cdot \varphi \equiv \sum_{T^{\prime} \in \mathcal{T}_{0}(\lambda)} r_{T^{\prime}} \varphi_{S T^{\prime}} \quad \bmod \mathcal{S}(\Lambda)^{\vee \lambda} .
$$

Since $\varphi_{S T} \in \mathcal{S}^{\mathbf{p}}$ and $\mathcal{S}(\Lambda)^{\vee \lambda} \cap \mathcal{S}^{\mathbf{p}}=\left(\mathcal{S}^{\mathbf{p}}\right)^{\vee(\lambda, 1)}$, the congruence relation by $\mathcal{S}(\Lambda)^{\vee \lambda}$ in the above formula can be replaced by $\left(\mathcal{S}^{\mathbf{p}}\right)^{\vee(\lambda, 1)}$. In particular, for $\varphi_{S T} \in$ 
$\mathcal{C}^{\mathbf{p}}(\lambda, 1), \varphi \in \mathcal{S}^{\mathbf{p}}$, we have

$$
\varphi_{S T} \cdot \varphi \equiv \sum_{T^{\prime} \in \mathcal{T}_{0}(\lambda)} r_{T^{\prime}} \varphi_{S T^{\prime}} \quad \bmod \left(\mathcal{S}^{\mathbf{p}}\right)^{\vee(\lambda, 1)} .
$$

On the other hand, by the second formula in Theorem 2.6 we have, for $\varphi_{S T} \in$ $\mathcal{C}^{\mathbf{p}}(\lambda, 0), \varphi \in \mathcal{S}^{\mathbf{p}}$

$$
\varphi_{S T} \cdot \varphi \equiv \sum_{T^{\prime} \in \mathcal{T}_{0}^{\mathbf{p}}(\lambda)} f_{T^{\prime}} \varphi_{S T^{\prime}} \quad \bmod \left(\mathcal{S}^{\mathbf{p}}\right)^{\vee(\lambda, 0)} ;
$$

but the first formula in Lemma 2.5 shows that $\varphi_{S T^{\prime}} \in \mathcal{C}^{\mathrm{P}}(\lambda, 1)$ does not appear in the expression of $\varphi_{S T} \cdot \varphi$ except $\varphi_{S T^{\prime}} \in \mathcal{C}^{\mathbf{p}}(\lambda, 0)$. It follows that the congruence relation $\left(\mathcal{S}^{\mathbf{p}}\right)^{\vee(\lambda, 0)}$ in the above formula can be replaced by $\left(\mathcal{S}^{\mathbf{p}}\right)^{\vee(\lambda, 1)}$. Thus we have, for $\varphi_{S T} \in \mathcal{C}^{\mathbf{p}}(\lambda, 0), \varphi \in \mathcal{S}^{\mathbf{p}}$,

$$
\varphi_{S T} \cdot \varphi \equiv \sum_{T^{\prime} \in \mathcal{T}_{0}^{\mathbf{p}}(\lambda)} f_{T^{\prime}} \varphi_{S T^{\prime}} \quad \bmod \left(\mathcal{S}^{\mathbf{p}}\right)^{\vee(\lambda, 1)} .
$$

We now prove (i). For $T \in \mathcal{T}_{0}^{\mathbf{p}}(\lambda), \varphi \in \mathcal{S}^{\mathbf{p}}$, one can write

$$
\begin{aligned}
& \varphi_{T}^{(\lambda, 0)} \cdot \varphi=\sum_{T^{\prime} \in \mathcal{T}_{0}^{\mathrm{p}}(\lambda)} g_{T^{\prime}} \varphi_{T^{\prime}}^{(\lambda, 0)}, \\
& \varphi_{T}^{(\lambda, 1)} \cdot \varphi=\sum_{T^{\prime} \in \mathcal{T}_{0}(\lambda)} g_{T^{\prime}}^{\prime} \varphi_{T^{\prime}}^{(\lambda, 1)} .
\end{aligned}
$$

By the definition of Weyl modules, we see that $g_{T^{\prime}}=f_{T^{\prime}}$ and $g_{T^{\prime}}^{\prime}=r_{T^{\prime}}$. Thus by comparing (3.5.1) and (3.5.2), we have

$$
g_{T^{\prime}}^{\prime}= \begin{cases}g_{T^{\prime}} & \text { if } T^{\prime} \in \mathcal{T}_{0}^{\mathbf{p}}(\lambda), \\ 0 & \text { otherwise }\end{cases}
$$

This proves (i). The assertion (ii) is proved in a similar way.

The following proposition can be proved in a similar way as in [Sa, Theorem 3.3].

Proposition 3.6. For each $\lambda \in \Lambda^{+}$, there exists an isomorphism of $\mathcal{S}(\Lambda)$-modules

$$
Z_{\mathbf{p}}^{(\lambda, 0)} \otimes_{\mathcal{S} \mathbf{p}} \mathcal{S}(\Lambda) \stackrel{\sim}{\rightarrow} W^{\lambda}
$$

which maps $\varphi_{T^{\lambda}}^{(\lambda, 0)} \psi \otimes \varphi$ to $\varphi_{T^{\lambda}} \psi \varphi$ for $\psi \in \mathcal{S}^{\mathbf{p}}, \varphi \in \mathcal{S}(\Lambda)$.

3.7. By Lemma 3.5, the map $\varphi_{T}^{(\lambda, 0)} \mapsto \varphi_{T}$ gives an injective homomorphism $f_{\lambda}: Z_{\mathbf{p}}^{(\lambda, 0)} \rightarrow W^{\lambda}$ of $\mathcal{S}^{\mathbf{p}}$-modules. By this map we regard $Z_{\mathbf{p}}^{(\lambda, 0)}$ as an $\mathcal{S}^{\mathbf{p}}$-submodule of $W^{\lambda}$. We have the following lemma.

Lemma 3.8. Assume that $\lambda \in \Lambda^{+}$.

(i) Let $M$ be an $\mathcal{S}^{\mathbf{p}}$-submodule of $Z_{\mathbf{p}}^{(\lambda, 0)}$, and let $\widetilde{M}$ be the $\mathcal{S}(\Lambda)$-submodule of $W^{\lambda}$ generated by $M$. Then $\widetilde{M} \cap Z_{\mathbf{p}}^{(\lambda, 0)}=M$.

(ii) Let $M_{1} \subsetneq M_{2}$ be $\mathcal{S}^{\mathbf{p}}$-submodules of $Z_{\mathbf{p}}^{(\lambda, 0)}$. Let $\iota_{i}$ be the inclusion map $M_{i} \rightarrow Z_{\mathbf{p}}^{(\lambda, 0)}$, and $\iota_{i} \otimes \operatorname{Id}$ be the induced map $M_{i} \otimes_{\mathcal{S}^{\mathbf{p}}} \mathcal{S}(\Lambda) \rightarrow Z_{\mathbf{p}}^{(\lambda, 0)} \otimes_{\mathcal{S}^{\mathrm{p}}} \mathcal{S}(\Lambda)$ for $i=1,2$. Then we have $\operatorname{Im}\left(\iota_{1} \otimes \operatorname{Id}\right) \subsetneq \operatorname{Im}\left(\iota_{2} \otimes \operatorname{Id}\right)$. 
Proof. We prove (i). Take $x \in \widetilde{M} \cap Z_{\mathbf{p}}^{(\lambda, 0)}$. We write $x=\sum_{T \in \mathcal{T}_{0}^{\mathbf{p}}(\lambda)} r_{T} \varphi_{T}^{(\lambda, 0)}$. Since $x \in \widetilde{M}$, one can write $x=\sum_{i} y_{i} \psi_{i}$ with $\psi_{i} \in \mathcal{S}(\Lambda), y_{i}=\sum_{T \in \mathcal{T}_{0}^{\mathrm{p}}(\lambda)} r_{T, i} \varphi_{T}^{(\lambda, 0)} \in$ $Z_{\mathbf{p}}^{(\lambda, 0)}$. Hence we have a relation as elements in $W^{\lambda}$,

$$
\sum_{T \in \mathcal{T}_{0}^{\mathbf{p}}(\lambda)} r_{T} \varphi_{T}=\sum_{i} \sum_{T \in \mathcal{T}_{0}^{\mathbf{p}}(\lambda)} r_{T, i} \varphi_{T} \psi_{i}
$$

This means that

$$
\sum_{T \in \mathcal{T}_{0}^{\mathbf{p}}(\lambda)} r_{T} \varphi_{T^{\lambda} T} \equiv \sum_{i} \sum_{T \in \mathcal{T}_{0}^{\mathbf{p}}(\lambda)} r_{T, i} \varphi_{T^{\lambda} T} \psi_{i} \quad \bmod \mathcal{S}(\Lambda)^{\vee \lambda}
$$

Put $\alpha=\alpha_{\mathbf{p}}(\lambda)$. Take $\nu \in \Lambda$ such that $\alpha_{\mathbf{p}}(\nu)=\alpha$ and multiply $\varphi_{\nu}$ on both sides of the above equation. Note that $\varphi_{\nu} \in \mathcal{S}^{\mathbf{p}}$ is a projection from $M$ to $M^{\nu}$, and we have $\mathcal{S}^{\mathbf{p}} \cap \mathcal{S}(\Lambda)^{\vee \lambda}=\left(\mathcal{S}^{\mathbf{p}}\right)^{\vee(\lambda, 1)} \subset\left(\mathcal{S}^{\mathbf{p}}\right)^{\vee(\lambda, 0)}$. It follows that

$$
\sum_{T \in \mathcal{T}_{0}(\lambda, \nu)} r_{T} \varphi_{T^{\lambda} T} \equiv \sum_{i} \sum_{T \in \mathcal{T}_{0}^{\mathbf{p}}(\lambda)} r_{T, i} \varphi_{T^{\lambda} T} \psi_{i} \varphi_{\nu} \quad \bmod \left(\mathcal{S}^{\mathbf{p}}\right)^{\vee(\lambda, 0)}
$$

Since this holds for any $\nu \in \Lambda$ such that $\alpha_{\mathbf{p}}(\nu)=\alpha$, we have

$$
\sum_{T \in \mathcal{T}_{0}^{\mathbf{p}}(\lambda)} r_{T} \varphi_{T^{\lambda} T} \equiv \sum_{\substack{\nu \in \Lambda \\ \alpha_{\mathbf{p}}(\nu)=\alpha}} \sum_{i} \sum_{T \in \mathcal{T}_{0}^{\mathbf{p}}(\lambda)} r_{T, i} \varphi_{T^{\lambda} T} \psi_{i} \varphi_{\nu} \quad \bmod \left(\mathcal{S}^{\mathbf{p}}\right)^{\vee(\lambda, 0)}
$$

Put $\varphi_{\alpha}=\sum_{\nu} \varphi_{\nu}$, where $\nu$ runs over all the elements in $\Lambda$ such that $\alpha_{\mathbf{p}}(\nu)=\alpha$. Since $\varphi_{\alpha}$ is the projection from $M$ onto $M^{\alpha}=\bigoplus_{\nu} M^{\nu}$, we see that $\varphi_{T^{\lambda} T} \varphi_{\alpha}=\varphi_{T^{\lambda} T}$ for any $T \in \mathcal{T}_{0}^{\mathbf{p}}(\lambda)$. Moreover, we note that $\varphi_{\alpha} \psi_{i} \varphi_{\nu} \in \mathcal{S}^{\mathbf{p}}$ since it is contained in $\operatorname{Hom}_{\mathcal{H}}\left(M^{\nu}, M^{\alpha}\right)$. It follows that

$$
\varphi_{T^{\lambda} T} \psi_{i} \varphi_{\nu}=\varphi_{T^{\lambda} T}\left(\varphi_{\alpha} \psi_{i} \varphi_{\nu}\right) \in \mathcal{S}^{\mathbf{p}}
$$

for $T \in \mathcal{T}_{0}^{\mathbf{p}}(\lambda)$. Thus one can rewrite (3.8.1) as a relation on $Z_{\mathbf{p}}^{(\lambda, 0)}$ as

$$
x=\sum_{T \in \mathcal{T}_{0}^{\mathbf{p}}(\lambda)} r_{T} \varphi_{T}^{(\lambda, 0)}=\sum_{\substack{\nu \in \Lambda \\ \alpha_{\mathbf{p}}(\nu)=\alpha}} \sum_{i} \sum_{T \in \mathcal{T}_{0}^{\mathbf{p}}(\lambda)} r_{T, i} \varphi_{T}^{(\lambda, 0)}\left(\varphi_{\alpha} \psi_{i} \varphi_{\nu}\right) .
$$

This shows that $x=\sum_{\nu} \sum_{i} y_{i}\left(\varphi_{\alpha} \psi_{i} \varphi_{\nu}\right) \in M$ as asserted.

Next we prove (ii). Under the embedding $Z_{\mathbf{p}}^{\lambda} \hookrightarrow W^{\lambda}$, Proposition 3.6 implies that $\operatorname{Im}\left(\iota_{i} \otimes \mathrm{Id}\right)=\widetilde{M}_{i}$. Take $x \in M_{2} \backslash M_{1}$. Suppose that $\widetilde{M}_{1}=\widetilde{M}_{2}$. Then $x \in$ $\widetilde{M}_{1} \cap Z_{\mathbf{p}}^{(\lambda, 0)}=M_{1}$ by (i). This is a contradiction.

By making use of Lemma 3.8, we show the following lemma.

Lemma 3.9. Assume that $R$ is a field. Then for each $\lambda \in \Lambda^{+}$, there exists a unique maximal $\mathcal{S}(\Lambda)$-submodule $N^{\lambda}$ of $L_{\mathbf{p}}^{(\lambda, 0)} \otimes_{\mathcal{S}^{\mathbf{p}}} \mathcal{S}(\Lambda)$ such that

$$
L_{\mathbf{p}}^{(\lambda, 0)} \otimes_{\mathcal{S}^{\mathrm{p}}} \mathcal{S}(\Lambda) / N^{\lambda} \simeq L^{\lambda} .
$$

Proof. Since $L_{\mathbf{p}}^{(\lambda, 0)} \simeq Z_{\mathbf{p}}^{(\lambda, 0)} / \operatorname{rad} Z_{\mathbf{p}}^{(\lambda, 0)}$, we have a surjective homomorphism

$$
Z_{\mathbf{p}}^{(\lambda, 0)} \otimes_{\mathcal{S}^{\mathbf{p}}} \mathcal{S}(\Lambda) \rightarrow L_{\mathbf{p}}^{(\lambda, 0)} \otimes_{\mathcal{S}^{\mathbf{p}}} \mathcal{S}(\Lambda)
$$

as $\mathcal{S}(\Lambda)$-modules. Since $L_{\mathbf{p}}^{(\lambda, 0)} \neq 0$, we have $L_{\mathbf{p}}^{(\lambda, 0)} \otimes_{\mathcal{S}^{\mathrm{p}}} \mathcal{S}(\Lambda) \neq 0$ by Lemma 3.8 , and the kernel of this map is a proper $\mathcal{S}(\Lambda)$-submodule of $Z_{\mathrm{p}}^{(\lambda, 0)} \otimes_{\mathcal{S}^{\mathrm{p}}} \mathcal{S}(\Lambda)$; but $Z_{\mathbf{p}}^{(\lambda, 0)} \otimes_{\mathcal{S}} \mathcal{S}(\Lambda)$ is isomorphic to $W^{\lambda}$ by Proposition 3.6, and $L^{\lambda} \simeq W^{\lambda} / \operatorname{rad} W^{\lambda}$. 
Since $\operatorname{rad} W^{\lambda}$ is the unique maximal submodule of $W^{\lambda}$, we see that there exists a surjective homomorphism $L_{\mathbf{p}}^{(\lambda, 0)} \otimes_{\mathcal{S} \mathbf{p}} \mathcal{S}(\Lambda) \rightarrow L^{\lambda}$ of $\mathcal{S}(\Lambda)$-modules. It is clear that $N^{\lambda}$ is the unique maximal submodule since it is a quotient of $\operatorname{rad} W^{\lambda}$.

Lemma 3.10. Assume that $R$ is a field. For each $\lambda \in \Lambda^{+}$, the $\mathcal{S}^{\mathbf{p}}$-module $L^{\lambda}$ contains $L_{\mathbf{p}}^{(\lambda, 0)}$ as a submodule.

Proof. By definition, we have $\beta_{\lambda}\left(\varphi_{S}^{(\lambda, 0)}, \varphi_{T}^{(\lambda, 0)}\right)=\left\langle\varphi_{S}, \varphi_{T}\right\rangle$ for any $S, T \in \mathcal{T}_{0}^{\mathbf{p}}(\lambda)$. Moreover, one can check that $\left\langle\varphi_{S}, \varphi_{T}\right\rangle=0$ for $S \in \mathcal{T}_{0}(\lambda) \backslash \mathcal{T}_{0}^{\mathbf{p}}(\lambda), T \in \mathcal{T}_{0}^{\mathbf{p}}(\lambda)$. It follows that $f_{\lambda}\left(\operatorname{rad} Z_{\mathbf{p}}^{(\lambda, 0)}\right) \subset \operatorname{rad} W^{\lambda}$, where $f_{\lambda}: Z_{\mathbf{p}}^{(\lambda, 0)} \hookrightarrow W^{\lambda}$ is the injective map given in 3.7. Then $f_{\lambda}$ induces a homomorphism $\bar{f}_{\lambda}: L_{\mathbf{p}}^{(\lambda, 0)} \rightarrow L^{\lambda}$ of $\mathcal{S}^{\mathbf{p}}$ modules. Since $f_{\lambda}\left(\varphi_{T^{\lambda}}^{(\lambda, 0)}\right)=\varphi_{T^{\lambda}} \notin \operatorname{rad} W^{\lambda}, \bar{f}_{\lambda}$ is a non-zero map. Since $L_{\mathbf{p}}^{(\lambda, 0)}$ is an irreducible $\mathcal{S}^{\mathbf{p}}$-module, $\bar{f}_{\lambda}$ is injective. This proves the lemma.

The following two results are generalizations of [Sa, Theorem 5.6, Theorem 5.7].

Proposition 3.11. Assume that $R$ is a field. Then for $\lambda, \mu \in \Lambda^{+}$,

$$
\left[Z_{\mathbf{p}}^{(\lambda, 0)}: L_{\mathbf{p}}^{(\mu, 0)}\right]_{\mathcal{S}^{\mathbf{p}}} \leq\left[W^{\lambda}: L^{\mu}\right]_{\mathcal{S}(\Lambda)} .
$$

Proof. We consider a composition series of $Z_{\mathbf{p}}^{(\lambda, 0)}$ as an $\mathcal{S}^{\mathbf{p}}$-module

$$
0=M_{0} \subsetneq M_{1} \subsetneq \cdots \subsetneq M_{k}=Z_{\mathbf{p}}^{(\lambda, 0)}
$$

such that $M_{j} / M_{j-1} \simeq L_{\mathbf{p}}^{\left(\mu_{j}, 0\right)}$. Let $i_{j}: M_{j} \hookrightarrow Z_{\mathbf{p}}^{(\lambda, 0)}$ be the inclusion map and $\iota_{j} \otimes \operatorname{Id}: M_{j} \otimes_{\mathcal{S}^{p}} \mathcal{S}(\Lambda) \rightarrow Z_{\mathbf{p}}^{(\lambda, 0)} \otimes_{\mathcal{S} \mathbf{p}} \mathcal{S}(\Lambda)$ be the induced map. Put $\mathcal{M}_{j}=\operatorname{Im}\left(\iota_{j} \otimes \operatorname{Id}\right)$. We have a filtration

$$
0=\mathcal{M}_{0} \subsetneq \mathcal{M}_{1} \subsetneq \cdots \subsetneq \mathcal{M}_{k}=Z_{\mathbf{p}}^{(\lambda, 0)} \otimes_{\mathcal{S} \mathbf{p}} \mathcal{S}(\Lambda) \simeq W^{\lambda}
$$

of $\mathcal{S}(\Lambda)$-submodules of $W^{\lambda}$ by Proposition 3.6 and Lemma 3.8. In order to prove the proposition, it is enough to show that $L^{\mu_{j}}$ occurs in the composition series of $\mathcal{M}_{j} / \mathcal{M}_{j-1}$ for each $j$. Since $M_{j} / M_{j-1} \simeq L_{\mathbf{p}}^{\left(\mu_{j}, 0\right)}$, we have the following diagram of $\mathcal{S}(\Lambda)$-modules:

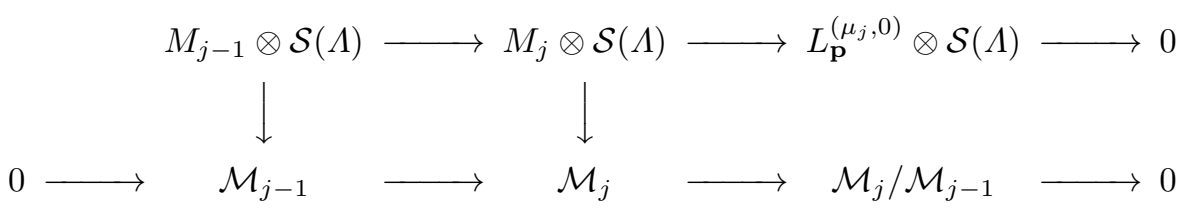

where the vertical maps are surjective. Thus we obtain a surjective homomorphism $L_{\mathbf{p}}^{(\lambda, 0)} \otimes_{\mathcal{S}^{\mathbf{P}}} \mathcal{S}(\Lambda) \rightarrow \mathcal{M}_{j} / \mathcal{M}_{j-1}$. On the other hand, by Lemma 3.9, we have a surjective homomorphism $L_{\mathbf{p}}^{(\lambda, 0)} \otimes_{\mathcal{S} \text { p }} \mathcal{S}(\Lambda) \rightarrow L^{\mu}$, whose kernel $N^{\lambda}$ is the unique maximal submodule. This implies that we have a surjective homomorphism $\mathcal{M}_{j} / \mathcal{M}_{j-1} \rightarrow L^{\mu_{j}}$. Hence $L^{\mu_{j}}$ occurs in the composition series of $\mathcal{M}_{j} / \mathcal{M}_{j-1}$, and the proposition is proved.

Proposition 3.12. Assume that $R$ is a field. Then for any $\lambda, \mu \in \Lambda^{+}$such that $\alpha_{\mathbf{p}}(\lambda)=\alpha_{\mathbf{p}}(\mu)$, we have

$$
\left[Z_{\mathbf{p}}^{(\lambda, 0)}: L_{\mathbf{p}}^{(\mu, 0)}\right]_{\mathcal{S}^{\mathbf{p}}} \geq\left[W^{\lambda}: L^{\mu}\right]_{\mathcal{S}(\Lambda)} .
$$


Proof. Consider a composition series of $W^{\lambda}$ as an $\mathcal{S}(\Lambda)$-module

$$
0=W_{0} \subsetneq W_{1} \subsetneq \cdots \subsetneq W_{k}=W^{\lambda}
$$

such that $W_{j} / W_{j-1} \simeq L^{\mu_{j}}$ for some $\mu_{j} \in \Lambda^{+}$. We consider this as a filtration of $W^{\lambda}$ as $\mathcal{S}^{\mathbf{p}}$-modules. Since $L^{\mu_{j}}$ contains $L_{\mathbf{p}}^{\left(\mu_{j}, 0\right)}$ as an $\mathcal{S}^{\mathbf{p}}$-submodule by Lemma 3.10 ,

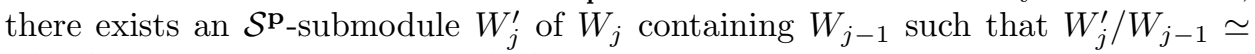
$L_{\mathbf{p}}^{\left(\mu_{j}, 0\right)}$. By 3.7, we identify $Z_{\mathbf{p}}^{(\lambda .0)}$ as an $\mathcal{S}^{\mathbf{p}}$-submodule of $W^{\lambda}$, and put $M_{j}=$ $Z_{\mathbf{p}}^{(\lambda, 0)} \cap W_{j}$ and $M_{j}^{\prime}=Z_{\mathbf{p}}^{(\lambda, 0)} \cap W_{j}^{\prime}$. We have a filtration of $Z_{\mathbf{p}}^{(\lambda, 0)}$ by $\mathcal{S}^{\mathbf{p}}$-modules

$$
0=M_{0} \subset M_{1}^{\prime} \subset M_{1} \subset \cdots \subset M_{k-1} \subset M_{k}^{\prime} \subset M_{k}=Z_{\mathbf{p}}^{(\lambda, 0)} \cap W^{\lambda}=Z_{\mathbf{p}}^{(\lambda, 0)} .
$$

We claim that

$$
M_{j-1} \neq M_{j}^{\prime} \quad \text { if } \quad \alpha_{\mathbf{p}}\left(\mu_{j}\right)=\alpha_{\mathbf{p}}(\lambda) .
$$

Note that (3.12.1) implies the proposition. In fact, $M_{j}^{\prime} / M_{j-1} \simeq L_{\mathbf{p}}^{\left(\mu_{j}, 0\right)}$ since it is isomorphic to a non-zero submodule of $L_{\mathbf{p}}^{\left(\mu_{j}, 0\right)}$. It follows that $L_{\mathbf{p}}^{\left(\mu_{j}, 0\right)}$ occurs in the composition series of $M_{j} / M_{j-1}$ for each $j$, and the proposition follows.

We show (3.12.1). Assume that $\alpha_{\mathbf{p}}\left(\mu_{j}\right)=\alpha_{\mathbf{p}}(\lambda)$. Then the image of $\varphi_{T^{\mu_{j}}}^{\left(\mu_{j}, 0\right)} \in$ $Z_{\mathbf{p}}^{\left(\mu_{j}, 0\right)}$ to $L_{\mathbf{p}}^{\left(\mu_{j}, 0\right)}$ gives a non-zero element $\bar{\varphi}_{j}$ in $L_{\mathbf{p}}^{\left(\mu_{j}, 0\right)}$ by Remark 2.9 (ii). We choose $x_{j} \in W_{j}^{\prime} \backslash W_{j-1}$ corresponding to $\bar{\varphi}_{j}$ under the isomorphism $W_{j}^{\prime} / W_{j-1} \simeq$ $L_{\mathbf{p}}^{\left(\mu_{j}, 0\right)}$. Since $\bar{\varphi}_{j} \varphi_{\mu_{j}}=\bar{\varphi}_{j}$, we have $x_{j} \varphi_{\mu_{j}} \in W_{j}^{\prime} \backslash W_{j-1}$. Since $x_{j} \in W_{j}^{\prime} \subset W^{\lambda}$, one can write $x_{j}=\sum_{T \in \mathcal{T}_{0}(\lambda)} r_{T} \varphi_{T}$. Now $\varphi_{\mu_{j}}$ is a projection from $M$ onto $M^{\mu_{j}}$. Hence

$$
x_{j} \varphi_{\mu_{j}}=\sum_{T \in \mathcal{T}_{0}^{\mathrm{P}}\left(\lambda, \mu_{j}\right)} r_{T} \varphi_{T} .
$$

Here $\mathcal{T}_{0}^{\mathbf{P}}\left(\lambda, \mu_{j}\right) \subset \mathcal{T}_{0}^{\mathbf{P}}(\lambda)=J_{\mathbf{p}}(\lambda, 0)$ since $\alpha_{\mathbf{p}}\left(\mu_{j}\right)=\alpha_{\mathbf{p}}(\lambda)$. It follows that the righthand side of the above equation is contained in $Z_{\mathbf{p}}^{(\lambda, 0)}$, and so $x_{j} \varphi_{\mu_{j}} \in M_{j}^{\prime} \backslash M_{j-1}$. This proves (3.12.1), and the proposition follows.

Combining Proposition 3.3, Proposition 3.11 and Proposition 3.12, we have the following theorem (cf. [Sa, Theorem 5.7]).

Theorem 3.13. Assume that $R$ is a field. For any $\lambda, \mu \in \Lambda^{+}$such that $\alpha_{\mathbf{p}}(\lambda)=$ $\alpha_{\mathbf{p}}(\mu)$, we have

$$
\left[\bar{Z}_{\mathbf{p}}^{\lambda}: \bar{L}_{\mathbf{p}}^{\mu}\right]_{\mathcal{S}^{\mathbf{p}}}=\left[Z_{\mathbf{p}}^{(\lambda, 0)}: L_{\mathbf{p}}^{(\mu, 0)}\right]_{\mathcal{S}^{\mathbf{p}}}=\left[W^{\lambda}: L^{\mu}\right]_{\mathcal{S}(\Lambda)} .
$$

\section{Structure theOrem For $\overline{\mathcal{S}}^{\mathbf{p}}$}

4.1. In this section, we assume that $\Lambda=\widetilde{\mathcal{P}}_{n, r}(\mathbf{m})$. For each $\mu \in \Lambda$, let $\widehat{N}^{\mathbf{a}_{\mathbf{p}}(\mu)}$ be the $R$-submodule of $\mathcal{H}$ spanned by $m_{\mathfrak{s t}}$ such that $\mathfrak{s}, \mathfrak{t} \in \operatorname{Std}(\lambda)$ with $\mathbf{a}_{\mathbf{p}}(\lambda)>\mathbf{a}_{\mathbf{p}}(\mu)$. Since $\lambda \unrhd \mu$ implies $\mathbf{a}_{\mathbf{p}}(\lambda) \geq \mathbf{a}_{\mathbf{p}}(\mu), \widehat{N}^{\mathbf{a}_{\mathbf{p}}(\mu)}$ is a two-sided ideal of $\mathcal{H}$. Put $\widehat{M}^{\mu}=$ $M^{\mu} \cap \widehat{N}^{\mathbf{a}_{\mathbf{p}}(\mu)}$. Then $\widehat{M}^{\mu}$ is an $\mathcal{H}$-module with the basis $\left\{m_{S \mathfrak{t}} \mid S \in \mathcal{T}_{0}(\lambda, \mu), \mathfrak{t} \in\right.$ $\left.\operatorname{Std}(\lambda), \mathbf{a}_{\mathbf{p}}(\lambda)>\mathbf{a}_{\mathbf{p}}(\mu)\right\}$. We define an $\mathcal{H}$-module $\bar{M}^{\mu}$ by $\bar{M}^{\mu}=M^{\mu} / \widehat{M}^{\mu}$ and let $f: M^{\mu} \rightarrow \bar{M}^{\mu}$ be the natural surjection. Put $\bar{m}_{S t}=f\left(m_{S t}\right)$ for a basis $m_{S t} \in M^{\mu}$. Then

$$
\left\{\bar{m}_{S \mathfrak{t}} \mid S \in \mathcal{T}_{0}^{\mathbf{p}}(\lambda, \mu), \mathfrak{t} \in \operatorname{Std}(\lambda) \text { for } \lambda \in \Lambda^{+}\right\}
$$

gives a basis of $\bar{M}^{\mu}$. 
4.2. We write $\mathbf{m}=\left(m_{1}, \ldots, m_{r}\right)$ in the form $\mathbf{m}=\left(\mathbf{m}^{[1]}, \ldots, \mathbf{m}^{[g]}\right)$ where $\mathbf{m}^{[k]}=$ $\left(m_{p_{k}+1}, \ldots, m_{p_{k}+r_{k}}\right)$. For each $n_{k} \in \mathbb{Z}_{\geq 0}$, put $\Lambda_{n_{k}}=\widetilde{\mathcal{P}}_{n_{k}, r_{k}}\left(\mathbf{m}^{[k]}\right)$ and $\Lambda_{n_{k}}^{+}=$ $\mathcal{P}_{n_{k}, r_{k}}\left(\mathbf{m}^{[k]}\right)$. $\quad\left(\Lambda_{n_{k}}\right.$ or $\Lambda_{n_{k}}^{+}$is regarded as the empty set if $n_{k}=0$.) Let $\mu=$ $\left(\mu^{(1)}, \ldots, \mu^{(r)}\right) \in \Lambda$ be an $r$-composition and write it as $\mu=\left(\mu^{[1]}, \ldots, \mu^{[g]}\right)$. Then a $\mu$-tableau $\mathfrak{t}=\left(\mathfrak{t}^{(1)}, \ldots, \mathfrak{t}^{(r)}\right)$ can be expressed as $\mathfrak{t}=\left(\mathfrak{t}^{[1]}, \ldots, \mathfrak{t}^{[g]}\right)$ with $\mathfrak{t}^{[k]}=$ $\left(\mathfrak{t}^{\left(p_{k}+1\right)}, \ldots, \mathfrak{t}^{\left(p_{k}+r_{k}\right)}\right)$, where $\mathfrak{t}^{[k]}$ is a $\mu^{[k]}$-tableau. Take $\lambda \in \Lambda^{+}, \mu \in \Lambda$ such that $\alpha_{\mathbf{p}}(\lambda)=\alpha_{\mathbf{p}}(\mu)$. Then a $\lambda$-tableau $T=\left(T^{(1)}, \ldots, T^{(r)}\right)$ of type $\mu$ can be expressed as $T=\left(T^{[1]}, \ldots, T^{[g]}\right)$ with $T^{[k]}=\left(T^{\left(p_{k}+1\right)}, \ldots, T^{\left(p_{k}+r_{k}\right)}\right)$, where $T^{[k]}$ is a $\lambda^{[k]}$-tableau of type $\mu^{[k]}$.

The following lemma is easily verified.

Lemma 4.3. Let $\alpha=\left(n_{1}, \ldots, n_{g}\right) \in \mathbb{Z}_{>0}^{g}$ be such that $n_{1}+\cdots+n_{g}=n$. Then:

(i) The map $\mu \mapsto\left(\mu^{[1]}, \ldots, \mu^{[g]}\right)$ gives a bijection between $\left\{\mu \in \Lambda \mid \alpha_{\mathbf{p}}(\mu)=\alpha\right\}$ and $\Lambda_{n_{1}} \times \cdots \times \Lambda_{n_{q}}$.

(ii) The map $\lambda \mapsto\left(\lambda^{[1]}, \ldots, \lambda^{[g]}\right)$ gives a bijection between $\left\{\lambda \in \Lambda^{+} \mid \alpha_{\mathbf{p}}(\lambda)=\right.$ $\alpha$ and $\Lambda_{n_{1}}^{+} \times \cdots \times \Lambda_{n_{g}}^{+}$.

(iii) For each $\lambda \in \Lambda^{+}, \mu \in \Lambda$ such that $\alpha_{\mathbf{p}}(\lambda)=\alpha_{\mathbf{p}}(\mu)$, the map $T \mapsto\left(T^{[1]}, \ldots\right.$, $\left.T^{[g]}\right)$ gives a bijection $\mathcal{T}_{0}^{\mathbf{p}}(\lambda, \mu) \simeq \mathcal{T}_{0}\left(\lambda^{[1]}, \mu^{[1]}\right) \times \cdots \times \mathcal{T}_{0}\left(\lambda^{[g]}, \mu^{[g]}\right)$.

4.4. Let $\alpha=\left(n_{1}, \ldots, n_{g}\right) \in \widetilde{\mathcal{P}}_{n, 1}$. For each $\lambda \in \Lambda^{+}$such that $\alpha_{\mathbf{p}}(\lambda)=\alpha$, we define a subset $\operatorname{Std}(\lambda)_{0}$ of $\operatorname{Std}(\lambda)$ as the set of $\mathfrak{t}=\left(\mathfrak{t}^{[1]}, \ldots, \mathfrak{t}^{[g]}\right)$ such that the letters contained in the tableau $\mathfrak{t}^{[k]}$ are exactly $\left\{n_{1}+\cdots+n_{k-1}+1, \ldots, n_{1}+\cdots+n_{k}\right\}$. Then the set $\operatorname{Std}(\lambda)_{0}$ is in bijection with the set $\operatorname{Std}\left(\lambda^{[1]}\right) \times \cdots \times \operatorname{Std}\left(\lambda^{[g]}\right)$ under the map $\mathfrak{t} \mapsto\left(\mathfrak{t}^{[1]}, \ldots, \mathfrak{t}^{[g]}\right)$. For each $\mu \in \Lambda$ such that $\alpha_{\mathbf{p}}(\mu)=\alpha$, we define an $R$-submodule $\bar{M}_{0}^{\mu}$ of $\bar{M}^{\mu}$ as the $R$-span of $\bar{m}_{S \mathfrak{t}}$ such that $S \in \mathcal{T}_{0}^{\mathbf{p}}(\lambda, \mu), \mathfrak{t} \in \operatorname{Std}(\lambda)_{0}$ for various $\lambda \in \Lambda^{+}$. We write $\mu=\left(\mu^{[1]}, \ldots, \mu^{[g]}\right)$ as before. Take $\mathfrak{s} \in \operatorname{Std}(\lambda) \operatorname{such}$ that $\mu(\mathfrak{s})=S$ for $S \in \mathcal{T}_{0}^{\mathbf{p}}(\lambda, \mu)$ with $\alpha_{\mathbf{p}}(\lambda)=\alpha_{\mathbf{p}}(\mu)=\alpha$. Then $\mathfrak{s} \in \operatorname{Std}(\lambda)_{0}$ and $\mathfrak{s}^{[k]} \in \operatorname{Std}\left(\lambda^{[k]}\right)$ has the property that $\mu^{[k]}\left(\mathfrak{s}^{[k]}\right)=S^{[k]}$. This gives a bijection between the set of $\mathfrak{s} \in \operatorname{Std}(\lambda)$ such that $\mu(\mathfrak{s})=S$ and the set of $\left(\mathfrak{s}^{[1]}, \ldots, \mathfrak{s}^{[g]}\right) \in$ $\operatorname{Std}\left(\lambda^{[1]}\right) \times \cdots \times \operatorname{Std}\left(\lambda^{[g]}\right)$ such that $\mu^{[k]}\left(\mathfrak{s}^{[k]}\right)=S^{[k]}$ for each $k$. Combined with (1.4.1) and (4.1.1), this implies that

(4.4.1) The map $\bar{m}_{S \mathfrak{t}} \mapsto m_{S^{[1]} \mathfrak{t}^{[1]}} \otimes \cdots \otimes m_{S^{[g]} \mathfrak{t}^{[g]}}$ gives an isomorphism of $R$-modules $\phi_{\mu}: \bar{M}_{0}^{\mu} \stackrel{\sim}{\rightarrow} M^{\mu^{[1]}} \otimes \cdots \otimes M^{\mu^{[g]}}$.

Put $\mathcal{H}_{\alpha}=\mathcal{H}_{n_{1}, r_{1}} \otimes \cdots \otimes \mathcal{H}_{n_{g}, r_{g}}$. Since $M^{\mu^{[k]}}$ is an $\mathcal{H}_{n_{k}, r_{k}}$-module, $M^{\mu^{[1]}} \otimes$ $\cdots \otimes M^{\mu^{[g]}}$ has a structure of an $\mathcal{H}_{\alpha}$-module. We denote by $T_{0}^{[k]}, \ldots, T_{n_{k}-1}^{[k]}$ the generators of $\mathcal{H}_{n_{k}, r_{k}}$ corresponding to $T_{0}, \ldots, T_{n-1}$ in the case of $\mathcal{H}_{n, r}$, and more generally we denote by $T_{w}^{[k]}$ for $w \in \mathfrak{S}_{n_{k}}$ the element corresponding to $T_{w} \in \mathcal{H}_{n}$. Then $T_{i}^{[k]}$ acts on $M^{\mu^{[1]}} \otimes \cdots \otimes M^{\mu^{[g]}}$ for $i=0, \ldots, n_{k}-1$, through the action of $1^{\otimes(k-1)} \otimes T_{i}^{[k]} \otimes 1^{\otimes(g-k)} \in \mathcal{H}_{\alpha}$ on it.

Recall that $L_{i}=T_{i-1} T_{i-2} \cdots T_{1} T_{0} T_{1} \cdots T_{i-2} T_{i-1} \in \mathcal{H}$ for $i=0, \ldots, n-1$. The following lemma is crucial for later discussions.

Lemma 4.5. Let $\mu \in \Lambda$ be such that $\alpha_{\mathbf{p}}(\mu)=\alpha$. For $\mu \in \Lambda$, put $\mathbf{a}_{\mathbf{p}}(\mu)=$ $\left(a_{1}, \ldots, a_{g}\right)$. Then the action of $L_{a_{k}+1}$ on $\bar{M}^{\mu}$ stabilizes the submodule $\bar{M}_{0}^{\mu}$, and it gives rise to the action of $T_{0}^{[k]}$ on $M^{\mu^{[1]}} \otimes \cdots \otimes M^{\mu^{[g]}}$ under the isomorphism $\phi_{\mu}$ in (4.4.1). 
Proof. Take $\lambda \in \Lambda^{+}$such that $\mathbf{a}_{\mathbf{p}}(\lambda)=\mathbf{a}_{\mathbf{p}}(\mu)$ and consider $\mathcal{T}_{0}^{\mathbf{p}}(\lambda, \mu)$. Let $\mathfrak{s} \in \operatorname{Std}(\lambda)$ such that $\mu(\mathfrak{s})=S$ for $S=\left(S^{[1]}, \ldots, S^{[g]}\right) \in \mathcal{T}_{0}^{\mathbf{p}}(\lambda, \mu)$. Then $\mathfrak{s}=\left(\mathfrak{s}^{[1]}, \ldots, \mathfrak{s}^{[g]}\right) \in$ $\operatorname{Std}(\lambda)_{0}$ and $\mu^{[k]}\left(\mathfrak{s}^{[k]}\right)=S^{[k]}$. Take $\mathfrak{s}$ as above, and take $\mathfrak{t}=\left(\mathfrak{t}^{[1]}, \ldots, \mathfrak{t}^{[g]}\right) \in \operatorname{Std}(\lambda)_{0}$. We consider a basis $m_{\mathfrak{s t}} \in \mathcal{H}$ and $m_{\mathfrak{s}^{[k]} \mathfrak{t}^{[k]}} \in \mathcal{H}_{n_{k}, r_{k}}$. We show that

(4.5.1) $m_{\mathfrak{s t}} L_{a_{k}+1}$ is written as a linear combination of the basis elements $m_{\mathfrak{u v}}$ of $\mathcal{H}$, where $\mathfrak{u}=\left(\mathfrak{u}^{[1]}, \ldots, \mathfrak{u}^{[g]}\right)$ is obtained from $\mathfrak{s}$ by replacing $\mathfrak{s}^{[k]}$ by some $\mathfrak{u}^{[k]}$, and $\mathfrak{v}$ is obtained from $\mathfrak{t}$ similarly. Here $\mathfrak{u}^{[k]}$ and $\mathfrak{v}^{[k]}$ have the same shape. The coefficient of $m_{\mathfrak{u v}}$ in the expansion of $m_{\mathfrak{s t}} L_{a_{k}+1}$ coincides with the coefficient of $m_{\mathfrak{u}^{[k]} \mathfrak{v}^{[k]}}$ in the expansion of $m_{\mathfrak{s}^{[k]}{ }_{[}^{[k]}} T_{0}^{[k]}$ under the bijection $\mathfrak{u} \leftrightarrow \mathfrak{u}^{[k]}, \mathfrak{v} \leftrightarrow \mathfrak{v}^{[k]}$.

(4.5.1) implies the lemma since $\mathfrak{u}, \mathfrak{v}$ are standard tableau of shape $\nu$ with $\mathbf{a}_{\mathbf{p}}(\nu)=$ $\mathbf{a}_{\mathbf{p}}(\mu)$ and $\mathfrak{v} \in \operatorname{Std}(\nu)_{0}$. We shall show (4.5.1). First we compute $m_{\mathfrak{s}^{[k]} \mathfrak{t}^{[k]}} T_{0}^{[k]}$ following the argument in the proof of [DJM, Proposition 3.20]. Recall that

$$
\lambda^{[k]}=\left(\lambda^{\left(p_{k}+1\right)}, \ldots, \lambda^{\left(p_{k}+r_{k}\right)}\right), \quad \mathfrak{t}^{[k]}=\left(\mathfrak{t}^{\left(p_{k}+1\right)}, \ldots, \mathfrak{t}^{\left(p_{k}+r_{k}\right)}\right),
$$

and put

$$
\begin{aligned}
& \beta=\left(\left|\lambda^{\left(p_{k}+1\right)}\right|, \ldots,\left|\lambda^{\left(p_{k}+r_{k}\right)}\right|\right)=\left(\beta_{1}, \ldots, \beta_{r_{k}}\right), \\
& \mathbf{b}=\left(b_{1}, \ldots, b_{r_{k}}\right) \text { with } b_{j}=\sum_{i=1}^{j-1} \beta_{i} .
\end{aligned}
$$

The letters contained in $\mathfrak{t}^{[k]}$ consist of $\left\{a_{k}+1, \ldots, a_{k}+n_{k}\right\}$. By the shift by $-a_{k}$, we regard $\mathfrak{t}^{[k]}$ as the tableau consisting of letters $\left\{1, \ldots, n_{k}\right\}$. Assume that the letter 1 is contained in $\mathfrak{t}^{\left(p_{k}+f\right)}$. One can write $d\left(\mathfrak{t}^{[k]}\right)=y c$, where $y \in \mathfrak{S}_{\beta}$ and $c$ is a distinguished coset representative in $\mathfrak{S}_{\beta} \backslash \mathfrak{S}_{n_{k}}$. Then $\mathfrak{t}^{\lambda^{[k]}} y$ is a standard tableau, and $c$ is a permutation which maps the letters $\left\{b_{i}+1, \ldots, b_{i}+\beta_{i}\right\}$ to the letters contained in $\mathfrak{t}^{\left(p_{k}+i\right)}$ for $i=1, \ldots, r_{k}$. Thus $y$ fixes the letter $b_{f}+1$, and $c$ can be expressed as $c=\left(b_{f+1}, b_{f}\right)\left(b_{f}, b_{f-1}\right) \cdots(2,1) c^{\prime}$, where $l(c)=b_{f}+l\left(c^{\prime}\right)$ and $c^{\prime}$ fixes the letter 1. It follows that $T_{c}^{[k]}=T_{b_{f}}^{[k]} T_{b_{f}-1}^{[k]} \cdots T_{2}^{[k]} T_{1}^{[k]} T_{c^{\prime}}^{[k]}$ and $T_{c^{\prime}}^{[k]} T_{0}^{[k]}=T_{0}^{[k]} T_{c^{\prime}}^{[k]}$. Recall that $m_{\mathfrak{s}^{[k]} \mathfrak{t}^{[k]}}=T_{d\left(\mathfrak{s}^{[k]}\right)}^{[k] *} m_{\lambda^{[k]}} T_{d\left(\mathfrak{t}^{[k]}\right)}^{[k]}$ with $m_{\lambda^{[k]}}=u_{\mathbf{b}}^{+} x_{\lambda^{[k]}}=x_{\lambda^{[k]}} u_{\mathbf{b}}^{+}$. Here $u_{\mathbf{b}}^{+}=u_{\mathbf{b}, 1} \cdots u_{\mathbf{b}, r_{k}}$, with

$$
u_{\mathbf{b}, j}=\prod_{i=1}^{b_{j}}\left(L_{i}^{[k]}-Q_{j}^{[k]}\right),
$$

where $L_{i}^{[k]}$ is the element in $\mathcal{H}_{n_{k}, r_{k}}$ corresponding to $L_{i} \in \mathcal{H}$, and $Q_{j}^{[k]}=Q_{p_{k}+j}$. Then as in the computation in DJM Prop. 3.20, Lemma 3.4], by noticing that $T_{y}^{[k]}$ commutes with $L_{b_{f}+1}^{[k]}$, we have

$$
\begin{aligned}
u_{\mathbf{b}}^{+} T_{y}^{[k]} T_{c}^{[k]} T_{0}^{[k]} & =u_{\mathbf{b}}^{+} T_{y}^{[k]} T_{b_{f}}^{[k]} T_{b_{f}-1}^{[k]} \cdots T_{1}^{[k]} T_{0}^{[k]} T_{c^{\prime}}^{[k]} \\
& =u_{\mathbf{b}}^{+} L_{b_{f}+1}^{[k]} T_{y}^{[k]}\left(T_{b_{f}}^{[k]}\right)^{-1} \cdots\left(T_{1}^{[k]}\right)^{-1} T_{c^{\prime}}^{[k]} \\
& =\left(Q_{f}^{[k]} u_{\mathbf{b}}^{+}+u_{\mathbf{b}^{\prime}}^{+}\right) T_{y}^{[k]}\left(T_{b_{f}}^{[k]}\right)^{-1} \cdots\left(T_{1}^{[k]}\right)^{-1} T_{c^{\prime}}^{[k]}
\end{aligned}
$$

where $\mathbf{b}^{\prime}=\left(b_{1}, \ldots, b_{f-1}, b_{f}+1, b_{f+1}, \ldots, b_{r_{k}}\right)$. It follows that

$$
m_{\mathfrak{s}^{[k]} \mathfrak{t}^{[k]}} T_{0}^{[k]}=T_{d\left(\mathfrak{s}^{[k]}\right)}^{[k] *} x_{\lambda^{[k]}}\left(Q_{f}^{[k]} u_{\mathbf{b}}^{+}+u_{\mathbf{b}^{\prime}}^{+}\right) T_{y}^{[k]} h,
$$


with

$$
h=\left(T_{b_{f}}^{[k]}\right)^{-1} \cdots\left(T_{1}^{[k]}\right)^{-1} T_{c^{\prime}}^{[k]} .
$$

Next we compute $m_{\mathfrak{s t}} L_{a_{k}+1}$ for $\mathfrak{s}, \mathfrak{t} \in \operatorname{Std}(\lambda)_{0}$. Recall that $m_{\mathfrak{s t}}=T_{d(\mathfrak{s})}^{*} m_{\lambda} T_{d(\mathfrak{t})}$ with $m_{\lambda}=x_{\lambda} u_{\mathbf{a}}^{+}$. Since $\mathfrak{t} \in \operatorname{Std}(\lambda)_{0}$, we have $d(\mathfrak{t})=d\left(\mathfrak{t}^{[1]}\right) \cdots d\left(\mathfrak{t}^{[g]}\right)$. (Note that the letters contained in $\mathfrak{t}^{[k]}$ consist of $\left\{a_{k}+1, \ldots, a_{k}+n_{k}\right\}$, and we compute $d\left(\mathfrak{t}^{[k]}\right)$ with respect to these letters.) We note that for $\mathfrak{t}=\left(\mathfrak{t}^{[1]}, \ldots, \mathfrak{t}^{[g]}\right)$, the letters contained in $\mathfrak{t}^{[1]}, \ldots, \mathfrak{t}^{[k-1]}$ consist of $\left\{1,2, \ldots, a_{k}\right\}$, and the letters contained in $\mathfrak{t}^{[k]}$ consist of $\left\{a_{k}+1, \ldots, a_{k}+n_{k}=a_{k+1}\right\}$, the letters contained in $\mathfrak{t}^{[k+1]}, \ldots, \mathfrak{t}^{[g]}$ consist of $\left\{a_{k+1}+1, \ldots, n\right\}$. It follows that

$$
\begin{aligned}
m_{\mathfrak{s t}} T_{a_{k}} T_{a_{k}-1} \cdots T_{1} T_{0}=T_{d(\mathfrak{s})}^{*} & x_{\lambda} u_{\mathbf{a}}^{+} T_{d(\mathfrak{t}[1])} \cdots T_{d\left(\mathfrak{t}^{[k]}\right)} \\
& \times T_{a_{k}} T_{a_{k}-1} \cdots T_{1} T_{0} T_{d\left(\mathfrak{t}^{[k+1]}\right)} \cdots T_{d(\mathfrak{t}[g]} .
\end{aligned}
$$

From the previous computation, we have $d\left(\mathfrak{t}^{[k]}\right)=y c$ with $y \in \mathfrak{S}_{\beta}$ and $c \in \mathfrak{S}_{\beta} \backslash \mathfrak{S}_{n_{k}}$. (Here we regard $\mathfrak{S}_{n_{k}}$ as the permutation group with respect to the letters $\left\{a_{k}+\right.$ $\left.1, \ldots, a_{k}+n_{k}\right\}$. In particular, $y$ fixes the letter $a_{k}+b_{f}+1$.) Hence

$$
T_{d(\mathfrak{t}[k])}=T_{y} T_{c}=T_{y} T_{a_{k}+b_{f}} T_{a_{k}+b_{f}-1} \cdots T_{a_{k}+1} T_{c^{\prime}} .
$$

Let $X$ be the left-hand side of (4.5.3). Since $T_{c^{\prime}}$ commutes with $T_{a_{k}}, \ldots, T_{1}, T_{0}$, we have

$$
\begin{aligned}
X=T_{d(\mathfrak{s})}^{*} & x_{\lambda} u_{\mathbf{a}}^{+} T_{d(\mathfrak{t}[1])} \cdots T_{d\left(\mathfrak{t}^{[k-1]}\right)} T_{y} \\
& \times T_{a_{k}+b_{f}} \cdots T_{a_{k}+1} T_{a_{k}} T_{a_{k}-1} \cdots T_{1} T_{0} T_{c^{\prime}} T_{d\left(\mathfrak{t}^{[k+1]}\right)} \cdots T_{d(\mathfrak{t}[g])} .
\end{aligned}
$$

Recall that $\mathbf{a}=\mathbf{a}(\lambda)=\left(a_{1}^{\prime}, \ldots, a_{r}^{\prime}\right)$ is defined by $a_{j}^{\prime}=\sum_{i=1}^{j-1}\left|\lambda^{(i)}\right|$, and $u_{\mathbf{a}}^{+}$is given by $u_{\mathbf{a}}^{+}=u_{\mathbf{a}, 1} u_{\mathbf{a}, 2} \cdots u_{\mathbf{a}, r}$, where $u_{\mathbf{a}, j}=\prod_{i=1}^{a_{j}^{\prime}}\left(L_{i}-Q_{j}\right)$. Hence $\mathbf{a}_{\mathbf{p}}=\left(a_{1}, \ldots, a_{g}\right)$ is given by $a_{i}=a_{p_{i}+1}^{\prime}$ for $i=1, \ldots, g$. Put

$$
u_{\mathbf{a}_{\mathbf{p}}, i}=u_{\mathbf{a}, p_{i}+1} \cdots u_{\mathbf{a}, p_{i}+r_{i}}
$$

for $i=1, \ldots, g$. Then we have $u_{\mathbf{a}}^{+}=u_{\mathbf{a}_{\mathbf{p}}, 1} \cdots u_{\mathbf{a}_{\mathbf{p}}, g}$ and $u_{\mathbf{a}_{\mathbf{p}}, k}, \ldots, u_{\mathbf{a}_{\mathbf{p}}, g}$ commutes with $T_{d\left(\mathfrak{t}^{[1]}\right)}, \ldots, T_{d\left(\mathfrak{t}^{[k-1]}\right)}$, and $u_{\mathbf{a}_{\mathbf{p}}, k+1}, \ldots, u_{\mathbf{a}_{\mathbf{p}}, g}$ commutes with $T_{y}$. It follows that

$$
\begin{aligned}
& u_{\mathbf{a}}^{+} T_{d\left(\mathfrak{t}^{[1]}\right)} \cdots T_{d\left(\mathfrak{t}^{[k-1]}\right)} T_{y} T_{a_{k}+b_{f}} \cdots T_{1} T_{0} \\
& \quad=u_{\mathbf{a}_{\mathbf{p}}, 1} \cdots u_{\mathbf{a}_{\mathbf{p}}, k-1} T_{d\left(\mathfrak{t}^{[1]}\right)} \cdots T_{d\left(\mathfrak{t}^{[k-1]}\right)} u_{\mathbf{a}_{\mathbf{p}}, k} T_{y} u_{\mathbf{a}_{\mathbf{p}}, k+1} \cdots u_{\mathbf{a}_{\mathbf{p}}, g} T_{a_{k}+b_{f}} \cdots T_{1} T_{0} .
\end{aligned}
$$

Since $u_{\mathbf{a}_{\mathbf{p}}, k+1}, \ldots, u_{\mathbf{a}_{\mathbf{p}}, g}$ commutes with $T_{a_{k}+b_{f}}, \ldots, T_{1}, T_{0}$, we have

$$
u_{\mathbf{a}_{\mathbf{p}}, k} T_{y} u_{\mathbf{a}_{\mathbf{p}}, k+1} \cdots u_{\mathbf{a}_{\mathbf{p}}, g} T_{a_{k}+b_{f}} \cdots T_{1} T_{0}=u_{\mathbf{a}_{\mathbf{p}}, k} T_{y} T_{a_{k}+b_{f}} \cdots T_{1} T_{0} u_{\mathbf{a}_{\mathbf{p}}, k+1} \cdots u_{\mathbf{a}_{\mathbf{p}}, g} .
$$

Since $T_{a_{k}+b_{f}} \cdots T_{1} T_{0}=L_{a_{k}+b_{f}+1} h^{\prime}$ with $h^{\prime}=T_{a_{k}+b_{f}}^{-1} \cdots T_{1}^{-1}$, and $T_{y}$ commutes with $L_{a_{k}+b_{f}+1}$, we have by [DJM, Lemma 3.4],

$$
\begin{aligned}
& u_{\mathbf{a}_{\mathbf{p}}, k} T_{y} T_{a_{k}+b_{f}} \cdots T_{1} T_{0} u_{\mathbf{a}_{\mathbf{p}}, k+1} \cdots u_{\mathbf{a}_{\mathbf{p}}, g} \\
& =\left(Q_{p_{k}+f} u_{\mathbf{a}_{\mathbf{p}}, k}+u_{\mathbf{a}_{\mathbf{p}}, k}^{\prime}\right) T_{y} h^{\prime} u_{\mathbf{a}_{\mathbf{p}}, k+1} \cdots u_{\mathbf{a}_{\mathbf{p}}, g},
\end{aligned}
$$

where $u_{\mathbf{a}_{\mathbf{p}}, k}^{\prime}$ is defined as in (4.5.5) by replacing $\mathbf{a}$ by

$$
\mathbf{a}^{\prime}=\left(a_{1}^{\prime}, \ldots, a_{p_{k}+f-1}^{\prime}, a_{p_{k}+f}^{\prime}+1, a_{p_{k}+f+1}^{\prime}, \ldots, a_{r}^{\prime}\right) .
$$

Summing up the above computation, we have

$$
\begin{aligned}
X=T_{d(\mathfrak{s})}^{*} & x_{\lambda} u_{\mathbf{a}_{\mathbf{p}}, 1} \cdots u_{\mathbf{a}_{\mathbf{p}}, k-1} T_{d\left(\mathfrak{t}^{[1]}\right)} \cdots T_{d\left(\mathfrak{t}^{[k-1]}\right)} \\
& \times\left(Q_{p_{k}+f} u_{\mathbf{a}_{\mathbf{p}}, k}+u_{\mathbf{a}_{\mathbf{p}}, k}^{\prime}\right) T_{y} h^{\prime} T_{c^{\prime}} u_{\mathbf{a}_{\mathbf{p}}, k+1} \cdots u_{\mathbf{a}_{\mathbf{p}}, g} T_{d\left(\mathfrak{t}^{[k+1]}\right)} \cdots T_{d\left(\mathfrak{t}^{[g]}\right)}
\end{aligned}
$$


It follows that

(4.5.6)

$$
\begin{aligned}
m_{\mathfrak{s t}} L_{a_{k}+1}= & X T_{1} \cdots T_{a_{k}}=T_{d(\mathfrak{s})}^{*} x_{\lambda} u_{\mathbf{a}_{\mathbf{p}}, 1} \cdots u_{\mathbf{a}_{\mathbf{p}}, k-1} T_{d\left(\mathfrak{t}^{[1]}\right)} \cdots T_{d\left(\mathfrak{t}^{[k-1]}\right)} \\
& \left.\times\left(Q_{p_{k}+f} u_{\mathbf{a}_{\mathbf{p}}, k}+u_{\mathbf{a}_{\mathbf{p}}, k}^{\prime}\right) T_{y} h^{\prime \prime} T_{c^{\prime}} u_{\mathbf{a}_{\mathbf{p}}, k+1} \cdots u_{\mathbf{a}_{\mathbf{p}}, g} T_{d\left(\mathfrak{t}^{[k+1]}\right)} \cdots T_{d(\mathfrak{t}[g]}\right)
\end{aligned}
$$

where $h^{\prime \prime}=T_{a_{k}+b_{f}}^{-1} \cdots T_{a_{k}+1}^{-1}$.

We now compare (4.5.2) and (4.5.6). The right-hand side of (4.5.2) is written as $X_{1}+X_{2}$, where $X_{1}=Q_{f}^{[k]} T_{d\left(\mathfrak{s}^{[k]}\right)}^{[k] *} x_{\lambda^{[k]}} u_{\mathbf{b}}^{+} T_{y}^{[k]} h$ and $X_{2}=T_{d(\mathfrak{s}[k])}^{[k] *} x_{\lambda^{[k]}} u_{\mathbf{b}^{\prime}}^{+} T_{y}^{[k]} h$. Since $x_{\lambda^{[k]}} u_{\mathbf{b}}^{+}=m_{\lambda^{[k]}}, X_{1}$ can be written, by Lemma 3.15 in [DJM], as a linear combination of the elements $m_{\mathfrak{s}^{[k]} \mathfrak{t}_{i}^{[k]}}$, where $\mathfrak{t}_{i}^{[k]}$ are row-standard tableaux. Then they are converted to a linear combination of the basis elements $m_{\mathfrak{u}[k] \mathfrak{v}[k]}$ in $\mathcal{H}_{n_{k}, r_{k}}$ by the procedure given in Proposition 3.18 in [loc. cit.], where $\mathfrak{u}^{[k]}, \mathfrak{v}^{[k]}$ are standard tableaux of shape $\mu^{[k]}$ for some $r_{k}$-partitions $\mu^{[k]}$. On the other hand, for $X_{2}$, first we convert $T_{d(\mathfrak{s}[k])}^{[k] *} x_{\lambda^{[k]}} u_{\mathbf{b}^{\prime}}^{+}$to a linear combination of the elements $m_{\mathfrak{u}_{1}^{[k]} \mathfrak{v}_{1}^{[k]}}$ where $\mathfrak{u}_{1}^{[k]}, \mathfrak{v}_{1}^{[k]}$ are row-standard tableau of shape $\nu^{[k]}\left(\nu^{[k]}\right.$ is determined from $\left.u_{\mathbf{b}^{\prime}}\right)$, and then we follow the argument in the case $X_{1}$. Note that in these computations, the parts $u_{\mathbf{b}}^{+}$and $u_{\mathbf{b}^{\prime}}^{+}$remain unchanged.

Next we consider (4.5.6). Since $T_{d(\mathfrak{s})}=T_{\left.d_{(\mathfrak{s}[1]}\right)} \cdots T_{d(\mathfrak{s}[g]}$ and $x_{\lambda}=x_{\lambda^{[1]}} \cdots x_{\lambda^{[g]}}$, one can write the formula (4.5.6) in the form

$$
m_{\mathfrak{s t}} L_{a_{k}+1}=Z \cdot T_{d(\mathfrak{s}[k]}^{*} x_{\lambda^{[k]}}\left(Q_{p_{k}+f} u_{\mathbf{a}_{\mathbf{p}}, k}+u_{\mathbf{a}_{\mathbf{p}}, k}^{\prime}\right) T_{y} h^{\prime \prime} T_{c^{\prime}} \cdot Z^{\prime}
$$

where

$$
\begin{aligned}
Z & =T_{d\left(\mathfrak{s}^{[1]}\right)}^{*} x_{\lambda^{[1]}} u_{\mathbf{a}_{\mathbf{p}}, 1} T_{d\left(\mathfrak{t}^{[1]}\right)} \cdots T_{d\left(\mathfrak{s}^{[k-1]}\right)}^{*} x_{\lambda^{[k-1]}} u_{\mathbf{a}_{\mathbf{p}}, k-1} T_{d\left(\mathfrak{t}^{[k-1]}\right)} \\
Z^{\prime} & =T_{d\left(\mathfrak{s}^{[k+1]}\right)}^{*} x_{\lambda^{[k+1]}} u_{\mathbf{a}_{\mathbf{p}}, k+1} T_{d\left(\mathfrak{t}^{[k+1]}\right)} \cdots T_{d\left(\mathfrak{s}^{[g]}\right)}^{*} x_{\lambda^{[g]}} u_{\mathbf{a}_{\mathbf{p}}, g} T_{d\left(\mathfrak{t}^{[g]}\right)} .
\end{aligned}
$$

Put

$$
\begin{aligned}
& Y_{1}=Q_{p_{k}+f} T_{d(\mathfrak{s}[k])}^{*} x_{\lambda^{[k]}} u_{\mathbf{a}_{\mathbf{p}}, k} T_{y} h^{\prime \prime} T_{c^{\prime}}, \\
& Y_{2}=T_{d(\mathfrak{s}[k])}^{*} x_{\lambda^{[k]}} u_{\mathbf{a}_{\mathbf{p}}, k}^{\prime} T_{y} h^{\prime \prime} T_{c^{\prime}},
\end{aligned}
$$

so that $m_{\mathfrak{s t}} L_{a_{k}+1}=Z\left(Y_{1}+Y_{2}\right) Z^{\prime}$. Let $\mathcal{H}_{n_{k}}^{\prime}$ be the subalgebra of $\mathcal{H}_{n}$ generated by $T_{a_{k}+1}, \cdots, T_{a_{k}+n_{k}-1}$. Then $T_{y}, T_{c^{\prime}}, h^{\prime \prime}$ belong to $\mathcal{H}_{n_{k}}^{\prime}$, and under the identification $\mathcal{H}_{n_{k}}^{\prime} \simeq \mathcal{H}_{n_{k}}, T_{y}, T_{c^{\prime}}$ coincide with $T_{y}^{[k]}, T_{c^{\prime}}^{[k]}$, and $h^{\prime \prime} T_{c^{\prime}}$ coincides with $h$. We also note that $Q_{p_{k}+f}=Q_{f}^{[k]}$. Now by applying Lemma 3.15 and Proposition 3.18 in [loc.cit], $Y_{1}$ can be expressed as a linear combination of the terms $T_{d\left(\mathfrak{u}^{[k]}\right)}^{*} x_{\mu^{[k]}} u_{\mathbf{a}_{\mathbf{p}}, k} T_{d(\mathfrak{v}[k])}$, where $\mathfrak{u}^{[k]}, \mathfrak{v}^{[k]}$ are standard tableaux of shape $\mu^{[k]}$ for some $r_{k}$-partitions $\mu^{[k]}$. Since this computation proceeds without referring $u_{\mathbf{a}_{\mathbf{p}}, k}$, the coefficients of these elements in the expansion of $Y_{1}$ are exactly the same as the coefficients of $m_{\mathfrak{u}^{[k] \mathfrak{v}[k]}}$ in the expansion of $X_{1}$. For $Y_{2}$, we first convert $T_{d(\mathfrak{s}[k]}^{*} x_{\lambda^{[k]}} u_{\mathbf{a}_{\mathbf{p}}, k}^{\prime}$ to a linear combination of the terms $T_{d\left(\mathfrak{u}_{1}^{[k]}\right)}^{*} x_{\nu[k]} u_{\mathbf{a}_{\mathbf{p}}, k}^{\prime} T_{d\left(\mathfrak{v}_{1}^{[k]}\right)}$ by using Proposition 3.20 in [loc.cit]. By comparing $\mathbf{b}^{\prime}$ and $\mathbf{a}^{\prime}$, we see that the coefficients in this expansion are exactly the same as the coefficients of $m_{\mathfrak{u}_{1}^{[k]} \mathfrak{v}_{1}^{[k]}}$ in the expansion of $T_{d\left(s^{[k]}\right)}^{[k] *} x_{\lambda^{[k]}} u_{\mathbf{b}^{\prime}}^{+}$. Thus again by applying Lemma 3.15 and Proposition 3.18 in [loc.cit], we conclude that $Y_{2}$ can be written as a linear combination of the terms $T_{d(\mathfrak{u}[k])}^{*} x_{\nu^{[k]}} u_{\mathbf{a}_{\mathbf{p}}, k}^{\prime} T_{d(\mathfrak{v}[k])}$, where $\mathfrak{u}^{[k]}, \mathfrak{v}^{[k]}$ are standard tableaux of shape $\nu^{[k]}$, and that their coefficients in the expansion of $Y_{2}$ is the same as the coefficients of $m_{\mathfrak{u}^{[k]} \mathfrak{v}^{[k]}}$ in the expansion of $X_{2}$. 
Now one sees easily that $Z \cdot T_{d\left(\mathfrak{u}^{[k]}\right)}^{*} x_{\mu^{[k]}} u_{\mathbf{a}_{\mathfrak{p}}, k} T_{d\left(\mathfrak{v}{ }^{[k]}\right)} \cdot Z^{\prime}=m_{\mathfrak{u v}}$, where $\mu$ is an $r$-partition obtained from $\lambda$ by replacing $\lambda^{[k]}$ by $\mu^{[k]}$, and $\mathfrak{u}, \mathfrak{v}$ are standard tableau of shape $\mu$ obtained from $\mathfrak{s}, \mathfrak{t}$ by replacing $\mathfrak{s}^{[k]}, \mathfrak{t}^{[k]}$ by $\mathfrak{u}^{[k]}, \mathfrak{v}^{[k]}$. A similar result holds also for $Z \cdot T_{d\left(\mathfrak{u}^{[k]}\right)}^{*} x_{\nu^{[k]}} u_{\mathbf{a}_{\mathbf{p}}, k}^{\prime} T_{d(\mathfrak{v}[k])} \cdot Z^{\prime}$. Summing up the above arguments, we see that (4.5.1) holds. Hence the lemma is proved.

The following lemma is easily verified by using a similar (but simpler) argument as in the proof of the previous lemma.

Lemma 4.6. Let the notation be as in Lemma 4.5. Then, for $i=1, \ldots, n_{k}-1$, the action of $T_{a_{k}+i}$ on $\bar{M}^{\mu}$ stabilizes $\bar{M}_{0}^{\mu}$, and it gives rise to the action of $T_{i}^{[k]}$ on $M^{\mu^{[1]}} \otimes \cdots \otimes M^{\mu^{[g]}}$ under the identification $\phi_{\mu}$ in (4.4.1).

4.7. We fix $\alpha=\left(n_{1}, \ldots, n_{g}\right) \in \widetilde{\mathcal{P}}_{n, 1}$, and let $\mathcal{H}_{\alpha}$ be as in 4.4. Assume that $\alpha_{\mathbf{p}}(\mu)=\alpha$ for $\mu \in \Lambda$. Then $\mathcal{H}_{\alpha}$ acts naturally on $M^{\mu^{[1]}} \otimes \cdots \otimes M^{\mu^{[g]}}$. Let $\mathbf{a}_{\mathbf{p}}(\mu)=\left(a_{1}, \ldots, a_{g}\right)$ be as before, and let $\widetilde{\mathcal{H}}_{\alpha}$ be the subalgebra of $\mathcal{H}$ generated by $T_{a_{k}+1}, \ldots, T_{a_{k}+r_{k}-1}, L_{a_{k}+1}$ for $k=1, \ldots, g$. As a corollary to Lemma 4.5 and Lemma 4.6, we have the following.

Corollary 4.8. For each $\mu \in \Lambda$ such that $\alpha_{\mathbf{p}}(\mu)=\alpha, \bar{M}_{0}^{\mu}$ is $\widetilde{H}_{\alpha}$-stable. The action of $\widetilde{H}_{\alpha}$ on $\bar{M}_{0}^{\mu}$ coincides with the action of $\mathcal{H}_{\alpha}$ on $M^{\mu^{[1]}} \otimes \cdots \otimes M^{\mu^{[g]}}$.

4.9. Recall that $\mathcal{S}=\bigoplus_{\mu, \nu \in \Lambda} \operatorname{Hom}_{\mathcal{H}}\left(M^{\nu}, M^{\mu}\right)$. It follows from the description of the basis of $\mathcal{S}^{\mathbf{p}}$ that

$$
\mathcal{S}^{\mathbf{p}}=\bigoplus_{\mu, \nu \in \Lambda} H_{\mu \nu}
$$

where $H_{\mu \nu}=\mathcal{S}^{\mathbf{p}} \cap \operatorname{Hom}_{\mathcal{H}}\left(M^{\nu}, M^{\mu}\right)$ is an $R$-submodule of $\operatorname{Hom}_{\mathcal{H}}\left(M^{\nu}, M^{\mu}\right)$ spanned by $\varphi_{S T}$ with $S \in \mathcal{T}_{0}(\lambda, \mu), T \in \mathcal{T}_{0}(\lambda, \nu)$ such that $\mathbf{a}_{\mathbf{p}}(\lambda)>\mathbf{a}_{\mathbf{p}}(\mu)$ if $\alpha_{\mathbf{p}}(\mu) \neq \alpha_{\mathbf{p}}(\nu)$. Then we have

$$
\overline{\mathcal{S}}^{\mathbf{p}}=\bigoplus_{\substack{\mu, \nu \in \Lambda \\ \alpha_{\mathbf{p}}(\mu)=\alpha_{\mathbf{p}}(\nu)}} \bar{H}_{\mu \nu},
$$

where $\bar{H}_{\mu \nu}=\pi\left(H_{\mu \nu}\right)$ is the $R$-span of the elements $\bar{\varphi}_{S T}$ such that $S \in \mathcal{T}_{0}^{\mathbf{p}}(\lambda, \mu)$, $T \in \mathcal{T}_{0}^{\mathbf{P}}(\lambda, \nu)$ for various $\lambda \in \Lambda^{+}$.

Assume that $\alpha_{\mathbf{p}}(\mu)=\alpha_{\mathbf{p}}(\nu)$. We claim that any $\varphi \in H_{\mu \nu}$ maps $\widehat{M}^{\nu}$ into $\widehat{M}^{\mu}$. In fact, take $\varphi \in H_{\mu \nu}$. Then by the property of $\varphi_{S T}$, there exists $h_{\varphi} \in \mathcal{H}$ such that $\varphi\left(m_{\nu} h\right)=h_{\varphi} m_{\nu} h$ for any $h \in \mathcal{H}$. Recall that $\widehat{M}^{\nu}$ is a linear combination of $m_{S \mathfrak{t}}$ with $S \in \mathcal{T}_{0}(\lambda, \nu), \mathfrak{t} \in \operatorname{Std}(\lambda)$ such that $\mathbf{a}_{\mathbf{p}}(\lambda)>\mathbf{a}_{\mathbf{p}}(\nu)$. Suppose that $m_{S t}$ is written as $m_{S t}=m_{\nu} h$ for some $h \in \mathcal{H}$. Then by the property of cellular basis, $\varphi\left(m_{S t}\right)=h_{\varphi} m_{S \mathfrak{t}}$ is a linear combination of $m_{\mathfrak{s}^{\prime} \mathfrak{t}^{\prime}}$, where $\mathfrak{s}^{\prime}, \mathfrak{t}^{\prime} \in \operatorname{Std}\left(\lambda^{\prime}\right)$ with $\lambda^{\prime} \unrhd \lambda$. Then we have $\mathbf{a}_{\mathbf{p}}\left(\lambda^{\prime}\right) \geq \mathbf{a}_{\mathbf{p}}(\lambda)>\mathbf{a}_{\mathbf{p}}(\nu)$. Since $\mathbf{a}_{\mathbf{p}}(\nu)=\mathbf{a}_{\mathbf{p}}(\mu)$, we have $\mathbf{a}_{\mathbf{p}}\left(\lambda^{\prime}\right)>\mathbf{a}_{\mathbf{p}}(\mu)$, and so $\varphi\left(m_{S t}\right) \in \widehat{M}^{\mu}$. Thus the claim holds.

By the claim, $\varphi$ induces a linear map $\bar{\varphi} \in \operatorname{Hom}_{\mathcal{H}}\left(\bar{M}^{\nu}, \bar{M}^{\mu}\right)$ under the condition that $\alpha_{\mathbf{p}}(\mu)=\alpha_{\mathbf{p}}(\nu)$. We note that $\bar{\varphi}=0$ if $\varphi \in \widehat{\mathcal{S}}^{\mathbf{p}}$. In fact, since $\mathbf{a}_{\mathbf{p}}(\mu)=\mathbf{a}_{\mathbf{p}}(\nu)$, we may consider the case where $\varphi=\varphi_{S T}$ for $S \in \mathcal{T}_{0}(\lambda, \mu), T \in \mathcal{T}_{0}(\lambda, \nu)$ with $\mathbf{a}_{\mathbf{p}}(\lambda) \neq \mathbf{a}_{\mathbf{p}}(\mu)$. Since $\lambda \unrhd \mu$, we have $\mathbf{a}_{\mathbf{p}}(\lambda)>\mathbf{a}_{\mathbf{p}}(\mu)$. It follows that $\varphi_{S T}\left(m_{\nu}\right)=$ $m_{S T} \in \widehat{M}^{\mu}$, and the image of $\varphi$ is contained in $\widehat{M}^{\mu}$. Hence $\bar{\varphi}=0$ as asserted. 
The above discussion allows us to define a linear map $\theta: H_{\mu \nu} \rightarrow \operatorname{Hom}_{\mathcal{H}}\left(\bar{M}^{\nu}, \bar{M}^{\mu}\right)$ by $\varphi \mapsto \bar{\varphi}$, which factors through the map $\bar{\theta}: \bar{H}_{\mu \nu} \rightarrow \operatorname{Hom}_{\mathcal{H}}\left(\bar{M}^{\nu}, \bar{M}^{\mu}\right)$. We show the following lemma.

Lemma 4.10. (i) For $\mu \in \Lambda$, let $\phi_{\mu}: \bar{M}_{0}^{\mu} \rightarrow M^{\mu^{[1]}} \otimes \cdots \otimes M^{\mu^{[g]}}$ be the isomorphism given in (4.4.1). Then we have

$$
\phi_{\mu}^{-1}\left(m_{\mu^{[1]}} \otimes \cdots \otimes m_{\mu[g]}\right)=\bar{m}_{\mu} .
$$

(ii) Assume that $\alpha_{\mathbf{p}}(\mu)=\alpha_{\mathbf{p}}(\nu)=\alpha$. Then for any $\varphi \in \bar{H}_{\mu \nu}, \bar{\varphi}=\bar{\theta}(\varphi)$ maps $\bar{M}_{0}^{\nu}$ to $\bar{M}_{0}^{\mu}$. In particular, $\bar{\varphi} \in \operatorname{Hom}_{\widetilde{\mathcal{H}}_{\alpha}}\left(\bar{M}_{0}^{\nu}, \bar{M}_{0}^{\mu}\right)$.

Proof. First we prove(i). Put $\mathbf{a}=\mathbf{a}(\mu)$ and $\mathbf{a}_{\mathbf{p}}=\mathbf{a}_{\mathbf{p}}(\mu)$. Then $m_{\mu}=x_{\mu} u_{\mathbf{a}}^{+}$, and $x_{\mu}=x_{\mu^{[1]}} \cdots x_{\mu[g]}, u_{\mathbf{a}}^{+}=u_{\mathbf{a}_{\mathbf{p}}, 1} \cdots u_{\mathbf{a}_{\mathbf{p}}, g}$, where $u_{\mathbf{a}_{\mathbf{p}}, i}$ is defined as in (4.5.5). One can write $m_{\mu}=x_{1} x_{2} \cdots x_{g}$ with $x_{k}=x_{\mu^{[k]}} u_{\mathbf{a}_{\mathbf{p}}, k}$. On the other hand, $m_{\mu^{[k]}}=$ $x_{\mu^{[k]}} u_{\mathbf{b}}^{+}$, where $\mathbf{b}=\mathbf{a}\left(\mu^{[k]}\right)$ is defined with respect to $\mu^{[k]} \in \widetilde{\mathcal{P}}_{n_{k}, r_{k}}\left(m^{[k]}\right)$. Then by Proposition 3.18 in [DJM], $m_{\mu^{[k]}}$ is written as a linear combination of the basis elements $m_{\mathfrak{u}^{[k]} \mathfrak{v}^{[k]}}$ of $\mathcal{H}_{n_{k}, r_{k}}$, where $\mathfrak{u}^{[k]}, \mathfrak{v}^{[k]}$ are standard tableau of shape $\lambda^{[k]}$. By the same procedure, $x_{k}$ is written as a linear combination of $x_{\mathfrak{u}^{[k]}} \mathfrak{v}^{[k]}=$ $T_{d(\mathfrak{u}[k])}^{*} x_{\lambda^{[k]}} u_{\mathbf{a}_{\mathbf{p}}, k} T_{d(\mathfrak{v}[k]}$, and the corresponding coefficient coincides with each other. Note that in the latter case $\mathfrak{u}^{[k]}, d\left(\mathfrak{u}^{[k]}\right)$, etc. are referred with respect to the letters $\left\{a_{k}+1, \ldots, a_{k}+n_{k}\right\}$ as in the proof of Lemma 4.5. We see that $x_{\mathfrak{u}^{[1]} \mathfrak{v}^{[1]}} \cdots x_{\mathfrak{u}^{[g]} \mathfrak{v}^{[g]}}$ gives rise to a basis element $m_{\mathfrak{u v}}$ of $\mathcal{H}$, where $\mathfrak{u}=\left(\mathfrak{u}^{[1]}, \ldots, \mathfrak{v}^{[g]}\right)$ and $\mathfrak{v}=\left(\mathfrak{v}^{[1]}, \ldots, \mathfrak{v}^{[g]}\right)$ are in $\operatorname{Std}(\lambda)_{0}$ with $\lambda=\left(\lambda^{[1]}, \ldots, \lambda^{[g]}\right)$. The assertion (i) follows from this.

Next we prove (ii). Now we have $\bar{m}_{\nu} \in \bar{M}_{0}^{\nu}$. Since $M^{\nu^{[1]}} \otimes \cdots \otimes M^{\nu^{[g]}}$ is generated by $m_{\nu^{[1]}} \otimes \cdots \otimes m_{\nu[g]}$ as an $\mathcal{H}_{\alpha}$-module, $\bar{M}_{0}^{\nu}$ is generated by $\bar{m}_{\nu}$ as an $\widetilde{H}_{\alpha}$-module. We take $\bar{\varphi}_{S T} \in \bar{H}_{\mu \nu}$. Then any element in $\bar{M}_{0}^{\nu}$ is written as $\bar{m}_{\nu} h$ with $h \in \widetilde{\mathcal{H}}_{\alpha}$, and $\varphi_{S T}\left(m_{\nu} h\right)=\varphi_{S T}\left(m_{\nu}\right) h=m_{S T} h$. Since $\bar{m}_{S T} \in \bar{M}_{0}^{\mu}$, we see that $\bar{\varphi}_{S T}\left(\bar{M}_{0}^{\nu}\right) \subseteq \bar{M}_{0}^{\mu}$. This proves (ii), and the lemma follows.

4.11. We keep the previous setting. By Lemma 4.10, one can define an $R$ linear map $\Theta: \bar{H}_{\mu \nu} \rightarrow \operatorname{Hom}_{\widetilde{\mathcal{H}}_{\alpha}}\left(\bar{M}_{0}^{\nu}, \bar{M}_{0}^{\mu}\right)$ induced from $\bar{\theta}$. On the other hand, in view of the isomorphisms $\phi_{\mu}, \phi_{\nu}$ together with Corollary 4.8, we have a natural isomorphism of $R$-modules:

$$
\begin{aligned}
& \operatorname{Hom}_{\tilde{\mathcal{H}}_{\alpha}}\left(\bar{M}_{0}^{\nu}, \bar{M}_{0}^{\mu}\right) \\
& \quad \simeq \operatorname{Hom}_{\mathcal{H}_{n_{1}, r_{1}}}\left(M^{\nu^{[1]}}, M^{\mu^{[1]}}\right) \otimes \cdots \otimes \operatorname{Hom}_{\mathcal{H}_{n_{g}, r_{g}}}\left(M^{\nu^{[g]}}, M^{\mu^{[g]}}\right) .
\end{aligned}
$$

We have the following lemma.

Lemma 4.12. The map $\Theta$ gives an isomorphism

$$
\bar{H}_{\mu \nu} \simeq \operatorname{Hom}_{\tilde{\mathcal{H}}_{\alpha}}\left(\bar{M}_{0}^{\nu}, \bar{M}_{0}^{\mu}\right)
$$

of $R$-modules. Let $\bar{\varphi}_{S T}$ be a basis element of $\bar{H}_{\mu \nu}$, where $S=\left(S^{[1]}, \ldots, S^{[g]}\right) \in$ $\mathcal{T}_{0}^{\mathbf{p}}(\lambda, \mu)$ and $T=\left(T^{[1]}, \ldots, T^{[g]}\right) \in \mathcal{T}_{0}^{\mathbf{p}}(\lambda, \nu)$ for some $\lambda \in \Lambda^{+}$. Then under the identification in (4.11.1), $\Theta$ maps $\bar{\varphi}_{S T}$ to $\varphi_{S^{[1]} T^{[1]}} \otimes \cdots \otimes \varphi_{S^{[g]} T^{[g]}}$.

Proof. It is enough to show the second assertion since $\varphi_{S^{[1]} T^{[1]}} \otimes \cdots \otimes \varphi_{S^{[g]} T^{[g]}}$ gives a basis of $\operatorname{Hom}_{\widetilde{\mathcal{H}}_{\alpha}}\left(\bar{M}_{0}^{\nu}, \bar{M}_{0}^{\mu}\right)$ under the identification in (4.11.1). Take $\bar{\varphi}_{S T} \in \bar{H}_{\mu \nu}$. Then $\bar{\varphi}_{S T}$ is defined by $\bar{\varphi}_{S T}\left(\bar{m}_{\nu}\right)=\bar{m}_{S T}$. By Lemma 4.10 (i), $\bar{m}_{\nu}$ is mapped to $m_{\nu^{[1]}} \otimes \cdots \otimes m_{\nu^{[g]}}$ via $\phi_{\nu} \cdot \bar{m}_{S T}$ is also mapped to $m_{S^{[1]} T^{[1]}} \otimes \cdots \otimes m_{S^{[g]} T^{[g]}}$ 
via $\phi_{\mu}$. Hence via the isomorphism (4.11.1), $\bar{\varphi}_{S T}$ corresponds to the $\mathcal{H}_{\alpha}$-linear map sending $m_{\nu^{[1]}} \otimes \cdots \otimes m_{\nu^{[g]}}$ to $m_{S^{[1]} T^{[1]}} \otimes \cdots \otimes m_{S^{[g]} T^{[g]}}$, which coincides with $\varphi_{S^{[1]} T^{[1]}} \otimes \cdots \otimes \varphi_{S^{[g]} T^{[g]}}$. The lemma is proved.

Remark 4.13. There exists an $R$-linear map $\psi: \bar{\theta}\left(\bar{H}_{\mu \nu}\right) \rightarrow \operatorname{Hom}_{\tilde{\mathcal{H}}_{\alpha}}\left(\bar{M}_{0}^{\nu}, \bar{M}_{0}^{\mu}\right)$ such that $\psi \circ \bar{\theta}=\Theta$ by Lemma 4.10. Hence $\bar{\theta}$ is injective by Lemma 4.12. However, $\bar{\theta}$ is not necessarily surjective. In Section 7 , we describe $\operatorname{Im} \bar{\theta}$ in terms of a modified Ariki-Koike algebra.

4.14. Let $\Delta_{n, g}$ be the set of $\alpha=\left(n_{1}, \ldots, n_{g}\right) \in \mathbb{Z}_{\geq 0}^{g}$ such that $n_{1}+\cdots+n_{g}=n$. For $\alpha \in \Delta_{n, g}$, put

$$
M^{\alpha}=\bigoplus_{\substack{\mu \in \Lambda \\ \alpha_{\mathbf{p}}(\mu)=\alpha}} M^{\mu}, \quad \bar{M}_{0}^{\alpha}=\bigoplus_{\substack{\mu \in \Lambda \\ \alpha_{\mathbf{p}}(\mu)=\alpha}} \bar{M}_{0}^{\mu} .
$$

Then $\mathcal{S}_{\alpha}^{\mathbf{p}}=\operatorname{End}_{\mathcal{H}}\left(M^{\alpha}\right)$ is a subalgebra of $\mathcal{S}^{\mathbf{p}}$, and we have $\mathcal{S}_{\alpha}^{\mathbf{p}}=\bigoplus_{\mu, \nu} H_{\mu \nu}$, where the sum is taken over all $\mu, \nu \in \Lambda$ such that $\alpha_{\mathbf{p}}(\mu)=\alpha_{\mathbf{p}}(\nu)=\alpha$. Put $\overline{\mathcal{S}}_{\alpha}^{\mathbf{p}}=\pi\left(\mathcal{S}_{\alpha}^{\mathbf{p}}\right)$. Then $\overline{\mathcal{S}}_{\alpha}^{\mathbf{p}}$ is a subalgebra of $\overline{\mathcal{S}}^{\mathbf{P}}$ such that $\overline{\mathcal{S}}_{\alpha}^{\mathbf{p}}=\bigoplus_{\mu, \nu} \bar{H}_{\mu \nu}$. Hence we have

$$
\overline{\mathcal{S}}^{\mathbf{p}}=\bigoplus_{\alpha \in \Delta_{n, g}} \overline{\mathcal{S}}_{\alpha}^{\mathbf{p}}
$$

On the other hand, Lemma 4.12 implies that

$$
\overline{\mathcal{S}}_{\alpha}^{\mathbf{p}} \simeq \operatorname{End}_{\widetilde{\mathcal{H}}_{\alpha}}\left(\bar{M}_{0}^{\alpha}\right) \text {. }
$$

We define an $\mathcal{H}_{n_{k}, r_{k}}$-module $M^{[k]}$ by $M^{[k]}=\bigoplus_{\mu^{[k]} \in \Lambda_{n_{k}}} M^{\mu^{[k]}}$. Define a cyclotomic $q$-Schur algebra $\mathcal{S}\left(\Lambda_{n_{k}}\right)$ associated to $\mathcal{H}_{n_{k}, r_{k}}$ by $\mathcal{S}\left(\Lambda_{n_{k}}\right)=\operatorname{End}_{\mathcal{H}_{n_{k}, r_{k}}} M^{[k]}$. Then we see that

$$
\operatorname{End}_{\mathcal{H}_{\alpha}}\left(\bigoplus_{\substack{\mu \in \Lambda \\ \alpha_{\mathbf{p}}(\mu)=\alpha}} M^{\mu^{[1]}} \otimes \cdots \otimes M^{\mu^{[g]}}\right) \simeq \mathcal{S}\left(\Lambda_{n_{1}}\right) \otimes \cdots \otimes \mathcal{S}\left(\Lambda_{n_{g}}\right) .
$$

The following structure theorem follows from (4.14.1)-(4.14.3) together with (4.11.1). Note that in the special case where $\mathbf{p}=\left(1^{r}\right)$, this result was proved in [SawS, Theorem 5.5 (i)] under the assumption that $Q_{i}-Q_{j}$ are units in $R$ for any $i \neq j$, and that $\Lambda=\widetilde{\mathcal{P}}_{n, r}(\mathbf{m})$ with $m_{i} \geq n$ for $i=1, \ldots, r$. In our case, we don't need any assumption for parameters $Q_{i}$ nor $\mathbf{m}$.

Theorem 4.15. Assume that $\Lambda=\widetilde{\mathcal{P}}_{n, r}(\mathbf{m})$. Then there exists an isomorphism of $R$-algebras

$$
\overline{\mathcal{S}}^{\mathbf{p}}(\Lambda) \simeq \bigoplus_{\substack{\left(n_{1}, \ldots, n_{g}\right) \\ n_{1}+\cdots+n_{g}=n}} \mathcal{S}\left(\Lambda_{n_{1}}\right) \otimes \cdots \otimes \mathcal{S}\left(\Lambda_{n_{g}}\right),
$$

where $\bar{\varphi}_{S T}$ is mapped to $\varphi_{S^{[1]} T^{[1]}} \otimes \cdots \otimes \varphi_{S^{[g]} T^{[g]}}$.

For $\lambda^{[k]}, \mu^{[k]} \in \Lambda_{n_{k}}^{+}$, let $W^{\lambda^{[k]}}$ be the Weyl module, and let $L^{\mu^{[k]}}$ be the irreducible module with respect to $\mathcal{S}\left(\Lambda_{n_{k}}\right)$. As a corollary to the previous theorem, we have

Corollary 4.16. Assume that $R$ is a field and $\Lambda$ is as above. Let $\lambda, \mu \in \Lambda^{+}$. Then under the isomorphism in Theorem 4.15, we have the following:

(i) $\bar{Z}_{\mathbf{p}}^{\lambda} \simeq W^{\lambda^{[1]}} \otimes \cdots \otimes W^{\lambda^{[g]}}$. 
(ii) $\bar{L}_{\mathbf{p}}^{\mu} \simeq L^{\mu^{[1]}} \otimes \cdots \otimes L^{\mu^{[g]}}$.

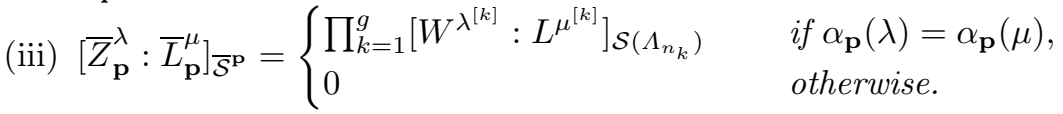

Combining this with Theorem 3.13, we have the following product formula for the decomposition numbers of $\mathcal{S}(\Lambda)$, which is a generalization of Sa, Corollary $5.10]$.

Theorem 4.17. Assume that $R$ is a field and that $\Lambda=\widetilde{\mathcal{P}}_{n, r}(\mathbf{m})$. For $\lambda, \mu \in \Lambda^{+}$ such that $\alpha_{\mathbf{p}}(\lambda)=\alpha_{\mathbf{p}}(\mu)$, we have

$$
\left[W^{\lambda}: L^{\mu}\right]_{\mathcal{S}(\Lambda)}=\prod_{k=1}^{g}\left[W^{\lambda^{[k]}}: L^{\mu[k]}\right]_{\mathcal{S}\left(\Lambda_{n_{k}}\right)} .
$$

\section{Modified Ariki-Koike algebra of type $\mathbf{p}$}

5.1. Throughout this section we assume the following property for $\mathbf{m}=\left(m_{1}, \ldots\right.$, $\left.m_{r}\right)$.

$$
m_{i} \geq n \text { for } i=1, \ldots, r .
$$

We keep the assumption that $\Lambda=\widetilde{\mathcal{P}}_{n, r}(\mathbf{m})$. Let $\Omega=\Omega^{\mathrm{p}}$ be a subset of $\Lambda$ consisting of $\omega=\left(\omega_{i}^{(j)}\right)=\left(\omega^{[1]}, \ldots, \omega^{[g]}\right)$ satisfying the properties

(i) $\omega_{i}^{(j)} \in\{0,1\}$,

(ii) $\sum_{j=1}^{r} \omega_{i}^{(j)}=1$ for $1 \leq i \leq n, 1 \leq j \leq r$,

(iii) $\omega_{i}^{(j)}=0$ unless $j=p_{1}+r_{1}, \ldots, p_{g}+r_{g}$. Hence $\omega^{[k]}=\left(-, \ldots,-, \omega^{\left(p_{k}+r_{k}\right)}\right)$ for $k=1, \ldots, g$.

Note that $\Omega$ coincides with $\Omega$ in $[\mathrm{SawS}, 7.1]$ in the case where $\mathbf{p}=\left(1^{r}\right)$ (i.e., the case $g=r$ ). While in the case where $\mathbf{p}=(r)$ (i.e., the case $g=1$ ), $\Omega=\{\omega\}$, where $\omega$ is an $r$-partition $\omega=\left(-, \ldots,-,\left(1^{n}\right)\right)$ which coincides with $\omega$ in [M, $\S 4$ ].

Let $I=\{1, \ldots, n\}$. For $\omega \in \Omega$, we denote by $I_{k}$ the set of $i$ such that $\omega_{i}^{\left(p_{k}+r_{k}\right)}=1$ for $k=1, \ldots, g$. Then $I=\coprod_{k=1}^{g} I_{k}$ gives a partition of $I$ into $g$ parts, and thanks to (5.1.1), the set $\Omega$ is in bijection with the set of partitions of $I$ into $g$ parts. For $\mathfrak{t}=\left(\mathfrak{t}^{[1]}, \ldots, \mathfrak{t}^{[t]}\right) \in \operatorname{Std}(\lambda)$, we denote by $I_{k}$ the letters contained in the standard tableau $\mathfrak{t}^{[k]}$. Then $I=\coprod I_{k}$ determines $\omega=\omega_{\mathfrak{t}} \in \Omega$. We associate to $\mathfrak{t}$ a semistandard tableau $T$ of shape $\lambda$ as follows: for each $k(1 \leq k \leq g)$, the first terms of the entries of $T^{\left(p_{k}+i\right)}$ consist of the entries of $\mathfrak{t}^{\left(p_{k}+i\right)}$, and the second term of them has the common value $p_{k}+r_{k}$ for $i=1, \ldots, r_{k}$. Then $T \in \mathcal{T}_{0}^{\mathbf{p}}(\lambda, \omega)$, and any element of $\mathcal{T}_{0}^{\mathrm{p}}(\lambda, \omega)$ is obtained from $\mathfrak{t} \in \operatorname{Std}(\lambda)$ such that $\omega=\omega_{\mathfrak{t}}$ by the above procedure. The correspondence $\mathfrak{t} \mapsto T$ gives a bijective correspondence

$$
\operatorname{Std}(\lambda) \simeq \bigcup_{\omega \in \Omega} \mathcal{T}_{0}^{\mathbf{p}}(\lambda, \omega)
$$

We denote by $\operatorname{Std}(\lambda)_{\omega}$ the subset of $\operatorname{Std}(\lambda)$ corresponding to $\mathcal{T}_{0}^{\mathbf{p}}(\lambda, \omega)$ under the bijection (5.1.2), i.e., $\operatorname{Std}(\lambda)_{\omega}=\left\{\mathfrak{t} \in \operatorname{Std}(\lambda) \mid \omega_{\mathfrak{t}}=\omega\right\}$.

Assume that $\omega \in \Omega$ corresponds to the partition $I=\coprod_{k} I_{k}$, where $\mathbf{a}_{\mathbf{p}}(\omega)=$ $\left(a_{1}, \ldots, a_{g}\right)$. We write $I_{k}$ as $I_{k}=\left\{i_{k 1}<i_{k 2}<\cdots<i_{k n_{k}}\right\}$. We define $d(\omega) \in \mathfrak{S}_{n}$ as

$$
d(\omega)=\left(\begin{array}{cccccc}
\ldots & a_{k}+1 & a_{k}+2 & \ldots & a_{k}+n_{k} & \ldots \\
\ldots & i_{k 1} & i_{k 2} & \ldots & i_{k n_{k}} & \ldots
\end{array}\right) .
$$


Suppose that $T \in \mathcal{T}_{0}^{\mathbf{p}}(\lambda, \omega)$ corresponds to $\mathfrak{t} \in \operatorname{Std}(\lambda)$ via (5.1.2). Let $\mathfrak{t}_{1} \in \operatorname{Std}(\lambda)$ be such that $\mathfrak{t}=\mathfrak{t}_{1} d(\omega)$. Then the letters contained in $\mathfrak{t}_{1}^{[k]}$ consist of $\left\{a_{k}+1, \ldots, a_{k}+\right.$ $\left.n_{k}\right\}$, and $\mathfrak{t}_{1}$ is the unique element in $\operatorname{Std}(\lambda)$ such that $\omega\left(\mathfrak{t}_{1}\right)=T$. In particular, assume that $S \in \mathcal{T}_{0}^{\mathbf{p}}(\lambda, \mu), T \in \mathcal{T}_{0}^{\mathbf{p}}(\lambda, \omega)$, for $\mu \in \Lambda, \omega \in \Omega$, and that $\mathfrak{t} \in \operatorname{Std}(\lambda)$ corresponds to $T$ via (5.1.2). Then we have

$$
m_{S T} T_{d(\omega)}=m_{S \mathrm{t}} .
$$

5.2. For each $\mu \in \Lambda$, let $\varphi_{\mu}$ be the identity map on $M^{\mu}$. By 2.4, $\varphi_{\mu} \in H_{\mu \mu}$, and we put $\bar{\varphi}_{\mu}=\pi\left(\varphi_{\mu}\right) \in \bar{H}_{\mu \mu}$. If we put $\bar{\varphi}_{\Omega}=\sum_{\omega \in \Omega} \bar{\varphi}_{\omega}, \bar{\varphi}_{\Omega}$ is an idempotent in $\overline{\mathcal{S}}^{\mathbf{p}}$, and we define a subalgebra $\overline{\mathcal{H}}^{\mathbf{p}}$ of $\overline{\mathcal{S}}^{\mathbf{p}}$ by $\overline{\mathcal{H}}^{\mathbf{p}}=\bar{\varphi}_{\Omega} \overline{\mathcal{S}}^{\mathbf{p}} \bar{\varphi}_{\Omega}$. We call $\overline{\mathcal{H}}^{\mathbf{p}}$ the modified Ariki-Koike algebra of type $\mathbf{p}$. In the case where $\mathbf{p}=\left(1^{r}\right), \overline{\mathcal{H}}^{\mathbf{p}}$ can be identified with the modified Ariki-Koike algebra given in [SawS] (see 7.1 in [loc. cit.]). One can write $\overline{\mathcal{H}}^{\mathbf{p}}=\bigoplus_{\omega, \omega^{\prime} \in \Omega} \bar{H}_{\omega \omega^{\prime}}$. In particular, $\overline{\mathcal{H}}^{\mathbf{p}}$ has an $R$-free basis

$$
\mathcal{B}^{\mathbf{p}}=\left\{\bar{\varphi}_{S T} \mid S \in \mathcal{T}_{0}^{\mathbf{p}}(\lambda, \omega), T \in \mathcal{T}_{0}^{\mathbf{p}}\left(\lambda, \omega^{\prime}\right) \text { for } \omega, \omega^{\prime} \in \Omega, \lambda \in \Lambda^{+}\right\} .
$$

Note that each $\bar{\varphi}_{S T} \in \mathcal{B}^{\mathbf{p}}$ determines uniquely the pair $\mathfrak{s}, \mathfrak{t}$ of standard tableau of shape $\lambda$ by (5.1.2). We denote $\bar{\varphi}_{S T}$ by $m_{\mathfrak{s t}}^{\mathbf{p}}$ if $S, T$ correspond to $\mathfrak{s}, \mathfrak{t} \in \operatorname{Std}(\lambda)$. Thus we see that

$$
\mathcal{B}^{\mathbf{p}}=\left\{m_{\mathfrak{s t}}^{\mathbf{p}} \mid \mathfrak{s}, \mathfrak{t} \in \operatorname{Std}(\lambda) \text { for some } \lambda \in \Lambda^{+}\right\} .
$$

Note that $\overline{\mathcal{S}}^{\mathbf{p}}$ has a structure of the cellular algebra with the cellular basis $\overline{\mathcal{C}}^{\mathbf{p}}$. Since the involution $*$ on $\overline{\mathcal{S}}^{\mathbf{p}}$ stabilizes the set $\mathcal{B}^{\mathrm{p}}$, we see that

$$
\overline{\mathcal{H}}^{\mathbf{p}} \text { is a cellular algebra with the cellular basis } \mathcal{B}^{\mathbf{p}} \text {. }
$$

More generally, we consider for each $\mu \in \Lambda$ an $R$-submodule $\bar{\varphi}_{\mu} \overline{\mathcal{S}}^{\mathbf{p}} \bar{\varphi}_{\Omega}$ of $\overline{\mathcal{S}}^{\mathbf{p}}$. Then $\bar{\varphi}_{\mu} \overline{\mathcal{S}}^{\mathbf{p}} \bar{\varphi}_{\Omega}$ has an $R$-basis

$$
\left\{\bar{\varphi}_{S T} \mid S \in \mathcal{T}_{0}^{\mathbf{p}}(\lambda, \mu), T \in \mathcal{T}_{0}^{\mathbf{p}}(\lambda, \omega) \text { for } \omega \in \Omega, \lambda \in \Lambda^{+}\right\} .
$$

Let $\bar{M}^{\Omega}=\bigoplus_{\omega \in \Omega} \bar{M}^{\omega}$, and put $\bar{m}_{\Omega}=\sum_{\omega \in \Omega} \bar{m}_{\omega} T_{d(\omega)} \in \bar{M}^{\Omega}$. Then for $S \in$ $\mathcal{T}_{0}^{\mathbf{p}}(\lambda, \mu), T \in \mathcal{T}_{0}^{\mathbf{p}}(\lambda, \omega)$, we have

$$
\bar{\varphi}_{S T}\left(\bar{m}_{\Omega}\right)=\bar{\varphi}_{S T}\left(\bar{m}_{\omega} T_{d(\omega)}\right)=\bar{m}_{S T} T_{d(\omega)}=\bar{m}_{S \mathfrak{t}}
$$

by (5.1.3), where $\mathfrak{t} \in \operatorname{Std}(\lambda)$ corresponds to $T$ via (5.1.2). Since $\left\{\bar{m}_{S \mathfrak{t}} \mid S \in\right.$ $\left.\mathcal{T}_{0}^{\mathbf{P}}(\lambda, \mu), \mathfrak{t} \in \operatorname{Std}(\lambda)\right\}$ gives a basis of $\bar{M}^{\mu}$, we see that the map $\varphi \mapsto \varphi\left(\bar{m}_{\Omega}\right)$ gives an isomorphism of $R$-modules

$$
\bar{\varphi}_{\mu} \overline{\mathcal{S}}^{\mathbf{p}} \bar{\varphi}_{\Omega} \simeq \bar{M}^{\mu}, \quad \bar{\varphi}_{S T} \leftrightarrow \bar{m}_{S \mathrm{t}} .
$$

Since $\bar{\varphi}_{\Omega} \overline{\mathcal{S}}^{\mathbf{p}} \bar{\varphi}_{\Omega}=\overline{\mathcal{H}}^{\mathbf{p}}$ acts naturally on $\bar{\varphi}_{\mu} \overline{\mathcal{S}}^{\mathbf{p}} \bar{\varphi}_{\Omega}$ from the right, one can define a right action of $\overline{\mathcal{H}}^{\mathbf{p}}$ on $\bar{M}^{\mu}$ through (5.2.4). Let $\mu, \nu \in \Lambda$ be such that $\alpha_{\mathbf{p}}(\mu)=\alpha_{\mathbf{p}}(\nu)$. By 4.9 , we know that $\varphi \in \bar{H}_{\mu \nu}$ gives a map $\bar{\theta}(\varphi)$ from $\bar{M}^{\nu}$ to $\bar{M}^{\mu}$. It is clear by definition, that $\bar{\theta}(\varphi)$ commutes with the action of $\overline{\mathcal{H}}^{\mathbf{p}}$. Hence we have an $R$-linear map $\theta^{\prime}: \bar{H}_{\mu \nu} \rightarrow \operatorname{Hom}_{\overline{\mathcal{H}}^{\mathbf{p}}}\left(\bar{M}^{\nu}, \bar{M}^{\mu}\right)$, which induces an $R$-algebra homomorphism $\theta^{\prime}: \overline{\mathcal{S}}^{\mathbf{p}} \rightarrow \operatorname{End}_{\overline{\mathcal{H}^{\mathrm{p}}}}(\bar{M})$, where $\bar{M}=\bigoplus_{\mu \in \Lambda} \bar{M}^{\mu}$.

The following result is a generalization of Proposition 7.5 in SawS. 
Proposition 5.3. For each $\alpha=\left(n_{1}, \ldots, n_{g}\right) \in \Delta_{n, g}$, put $n_{\alpha}=n ! / n_{1} ! \cdots n_{g} !$. Then we have an isomorphism of $R$-algebras

$$
\overline{\mathcal{H}}^{\mathbf{p}} \simeq \bigoplus_{\alpha \in \Delta_{n, g}} M_{n_{\alpha}}\left(\mathcal{H}_{n_{1}, r_{1}} \otimes \cdots \otimes \mathcal{H}_{n_{g}, r_{g}}\right) .
$$

Proof. By (4.14.1), one can write

$$
\overline{\mathcal{H}}^{\mathbf{p}}=\bar{\varphi}_{\Omega} \overline{\mathcal{S}}^{\mathbf{p}} \bar{\varphi}_{\Omega}=\bigoplus_{\alpha \in \Lambda_{n, g}} \bar{\varphi}_{\Omega, \alpha} \overline{\mathcal{S}}_{\alpha}^{\mathbf{p}} \bar{\varphi}_{\Omega, \alpha} .
$$

Here $\bar{\varphi}_{\Omega, \alpha}=\sum_{\omega} \bar{\varphi}_{\omega}$ is an idempotent of $\overline{\mathcal{S}}_{\alpha}^{\mathbf{p}}$, where the sum is taken over all $\omega \in \Omega$ such that $\alpha_{\mathbf{p}}(\omega)=\alpha$. We define a subalgebra $\overline{\mathcal{H}}_{\alpha}^{\mathbf{p}}$ of $\overline{\mathcal{H}}^{\mathbf{p}}$ by $\overline{\mathcal{H}}_{\alpha}^{\mathbf{p}}=\bar{\varphi}_{\Omega, \alpha} \overline{\mathcal{S}}_{\alpha}^{\mathbf{p}} \bar{\varphi}_{\Omega, \alpha}$. Put $\bar{M}_{0}^{\Omega, \alpha}=\bar{M}^{\Omega} \cap \bar{M}_{0}^{\alpha}$. Then by (4.14.2) we have

$$
\overline{\mathcal{H}}_{\alpha}^{\mathbf{p}} \simeq \operatorname{End}_{\tilde{\mathcal{H}}_{\alpha}}\left(\bar{M}_{0}^{\Omega, \alpha}\right)=\bigoplus_{\substack{\omega, \omega^{\prime} \in \Omega \\ \alpha_{\mathbf{p}}(\omega)=\alpha_{\mathbf{p}}\left(\omega^{\prime}\right)=\alpha}} \operatorname{Hom}_{\tilde{\mathcal{H}}_{\alpha}}\left(\bar{M}_{0}^{\omega}, \bar{M}_{0}^{\omega^{\prime}}\right) .
$$

Now the $\widetilde{\mathcal{H}}_{\alpha}$-module $\bar{M}_{0}^{\omega}$ is isomorphic to the $\mathcal{H}_{\alpha}$-module $M^{\omega^{[1]}} \otimes \cdots \otimes M^{\omega^{[g]}}$ by Corollary 4.8. In our case $M^{\omega^{[k]}}=\mathcal{H}_{n_{k}, r_{k}}$ (see 5.1). Hence for any $\omega, \omega^{\prime} \in \Omega$ such that $\alpha_{\mathbf{p}}(\omega)=\alpha_{\mathbf{p}}\left(\omega^{\prime}\right)=\alpha$, we have

$$
\begin{aligned}
\operatorname{Hom}_{\widetilde{\mathcal{H}}_{\alpha}}\left(\bar{M}_{0}^{\omega}, \bar{M}_{0}^{\omega^{\prime}}\right) & \simeq \operatorname{End}_{\mathcal{H}_{\alpha}}\left(\mathcal{H}_{n_{1}, r_{1}} \otimes \cdots \otimes \mathcal{H}_{n_{g}, r_{g}}\right) \\
& \simeq \mathcal{H}_{n_{1}, r_{1}} \otimes \cdots \otimes \mathcal{H}_{n_{g}, r_{g}} .
\end{aligned}
$$

The proposition follows from this by noticing that $\sharp\left\{\omega \in \Omega \mid \alpha_{\mathbf{p}}(\omega)=\alpha\right\}=n_{\alpha}$.

5.4. By $\bar{\theta}, \overline{\mathcal{S}}^{\mathrm{p}}$ acts on $\bar{M}$ from the left, and which commutes with the right action of $\mathcal{H}$. Hence we have a homomorphism $\rho: \mathcal{H} \rightarrow \operatorname{End}_{\mathcal{\mathcal { S }}^{\mathrm{p}}}^{0} \bar{M}$ (see Notation). Since $\sum_{\mu \in \Lambda} \bar{\varphi}_{\mu}=\operatorname{Id}_{\bar{M}}$, we have $\overline{\mathcal{S}}^{\mathrm{p}} \bar{\varphi}_{\Omega} \simeq \bar{M}$ by (5.2.4). This implies a natural isomorphism of $R$-algebras

$$
\operatorname{End} \frac{0}{\overline{\mathcal{S}}^{\mathbf{p}}} \bar{M} \simeq \operatorname{End} \frac{0}{\mathcal{S}}\left(\overline{\mathcal{S}}^{\mathbf{p}} \bar{\varphi}_{\Omega}\right) \simeq \bar{\varphi}_{\Omega} \overline{\mathcal{S}}^{\mathbf{p}} \bar{\varphi}_{\Omega}=\overline{\mathcal{H}}^{\mathbf{p}},
$$

where the second isomorphism is given by $f \mapsto f\left(\bar{\varphi}_{\Omega}\right)$ for $f \in \operatorname{End}_{\overline{\mathcal{S}}^{\mathrm{p}}}\left(\overline{\mathcal{S}}^{\mathbf{p}} \bar{\varphi}_{\Omega}\right)$. It follows that we have a homomorphism $\rho_{0}: \mathcal{H} \rightarrow \overline{\mathcal{H}}^{\mathbf{p}}$ of $R$-algebras thorough $\mathcal{H} \rightarrow \operatorname{End} \frac{0}{\mathcal{S}^{\mathrm{p}}} \bar{M}$. The homomorphism $\rho_{0}$ is explicitly given as follows: we have $\overline{\mathcal{H}}^{\mathbf{p}}=\bar{\varphi}_{\Omega} \overline{\mathcal{S}}^{\mathbf{p}} \bar{\varphi}_{\Omega} \simeq \bar{M}^{\Omega}$ via $\varphi \mapsto \varphi\left(\bar{m}_{\Omega}\right)$. Then for each $h \in \mathcal{H}$, there exists a unique $\varphi_{h} \in \overline{\mathcal{H}}^{\mathbf{p}}$ such that $\varphi_{h}\left(\bar{m}_{\Omega}\right)=\bar{m}_{\Omega} h \in \bar{M}^{\Omega}$. The map $h \mapsto \varphi_{h}$ gives $\rho_{0}$.

Now $\overline{\mathcal{H}}^{\mathbf{p}}$-module $\bar{M}$ is regarded as an $\mathcal{H}$-module via $\rho_{0}$, which coincides with the original $\mathcal{H}$-module $\bar{M}$. It follows that we have an injection

$$
\operatorname{Hom}_{\overline{\mathcal{H}}^{\mathbf{p}}}\left(\bar{M}^{\nu}, \bar{M}^{\mu}\right) \hookrightarrow \operatorname{Hom}_{\mathcal{H}}\left(\bar{M}^{\nu}, \bar{M}^{\mu}\right),
$$

and $\bar{\theta}$ factors through $\theta^{\prime}$ via this injection. Since $\bar{\theta}$ is injective by Remark 4.13, we see that

(5.4.2) The map $\theta^{\prime}: \bar{H}_{\mu \nu} \rightarrow \operatorname{Hom}_{\overline{\mathcal{H}}^{\mathbf{p}}}\left(\bar{M}^{\nu}, \bar{M}^{\mu}\right)$ is injective.

Since $\bar{M}^{\mu}$ is generated by $\bar{m}_{\mu}$ as an $\mathcal{H}$-module, it is generated by $\bar{m}_{\mu}$ as an $\overline{\mathcal{H}}^{\mathbf{p}}$ module, i.e., we have $\bar{M}^{\mu}=\bar{m}_{\mu} \overline{\mathcal{H}}^{\mathbf{P}}$. The following lemma is also clear from the fact that $\overline{\mathcal{H}}^{\mathbf{p}} \simeq \bar{M}^{\Omega}$ via $\varphi \mapsto \varphi\left(\bar{m}_{\Omega}\right)$ as noticed above. 
Lemma 5.5. We have $\bar{M}^{\Omega}=\bar{m}_{\Omega} \overline{\mathcal{H}}^{\mathbf{p}}$. The map $h \mapsto \bar{m}_{\Omega} h$ gives an isomorphism of $R$-modules $\overline{\mathcal{H}}^{\mathbf{P}} \rightarrow \bar{M}^{\Omega}$, namely $\bar{M}^{\Omega}$ is the regular representation of $\overline{\mathcal{H}}^{\mathbf{P}}$.

\section{Presentation for $\overline{\mathcal{H}}^{\mathbf{p}}$}

6.1 We shall define several elements in $\overline{\mathcal{H}}^{\mathbf{p}}$, and show that they generate $\overline{\mathcal{H}}^{\mathbf{p}}$. For each $\omega \in \Omega$ let $I=\coprod I_{k}$ be the corresponding partition of $I$. Define a map $b_{\omega}: I \rightarrow \mathbb{Z}_{>0}$ by $b_{\omega}(i)=k$ if $i \in I_{k}$. We put $Q_{k}^{\mathbf{p}}=Q_{p_{k}+r_{k}}$ for $k=1, \ldots, g$. Under this notation, we define elements $\xi_{i} \in \overline{\mathcal{S}}^{\mathrm{p}}$, for $i=1, \ldots, n$, by

$$
\xi_{i}=\sum_{\omega \in \Omega} Q_{b_{\omega}(i)}^{\mathbf{p}} \bar{\varphi}_{\omega}
$$

Clearly, $\bar{\varphi}_{\Omega} \xi_{i} \bar{\varphi}_{\Omega}=\xi_{i}$, and so $\xi_{1}, \ldots, \xi_{n}$ are elements in $\overline{\mathcal{H}}^{\mathbf{p}}$. They commute each other. Moreover, they satisfy the relation

$$
\left(\xi_{j}-Q_{1}^{\mathbf{p}}\right)\left(\xi_{j}-Q_{2}^{\mathbf{p}}\right) \cdots\left(\xi_{j}-Q_{g}^{\mathbf{p}}\right)=0
$$

for $j=1, \ldots, n$.

Under the isomorphism in (5.2.4), the action of $\xi_{i}$ on the basis element $\bar{m}_{S t}$ in $\bar{M}^{\mu}$ is given as follows:

$$
\bar{m}_{S \mathfrak{t}} \xi_{i}=Q_{b_{\omega}(i)}^{\mathbf{p}} \bar{m}_{S \mathfrak{t}} \quad \text { if } \mathfrak{t} \in \operatorname{Std}(\lambda)_{\omega}
$$

where $\operatorname{Std}(\lambda)_{\omega}$ is as in 5.1. Note that in this case $b_{\omega}(i)$ coincides with $k$ such that the letter $i$ is contained in $\mathfrak{t}^{[k]}$. By [DJM, Proposition 3.18], $\bar{m}_{\mu}$ is written, for $\mu \in \Lambda$, as a linear combination of $\bar{m}_{\mathfrak{s t}}$ such that the letters contained in the $k$ component of $\mathfrak{t}$ is the same as that of $\mathfrak{t}^{\mu}$. It follows from this, by making use of (6.1.3), that

$$
\bar{m}_{\mu} \xi_{i}=Q_{b(i)}^{\mathrm{p}} \bar{m}_{\mu},
$$

where $b(i)=k$ if $a_{k}+1 \leq i \leq a_{k}+n_{k}$ under the notation $\mathbf{a}_{\mathbf{p}}(\mu)=\left(a_{1}, \ldots, a_{g}\right)$ and $\alpha_{\mathbf{p}}(\mu)=\left(n_{1}, \ldots, n_{g}\right)$.

Let $\rho_{0}: \mathcal{H} \rightarrow \overline{\mathcal{H}}^{\mathbf{p}}$ be the homomorphism defined in 5.4. We note that

(6.1.5) The restriction of $\rho_{0}$ on $\mathcal{H}_{n}$ is injective.

In fact, it is enough to show that $\rho_{0}\left(T_{w}\right)\left(w \in \mathfrak{S}_{n}\right)$ are linearly independent as operators on $\bar{M}$. Now $\bar{M}=\bigoplus_{\alpha \in \Delta_{n, g}} \bar{M}^{\alpha}$, and $T_{w}$ preserves the subspaces $\bar{M}^{\alpha}$. We choose $\alpha$ such that $\alpha=(n, 0, \ldots, 0)$. Then $\mathcal{H}_{n}$ is contained in $\widetilde{\mathcal{H}}_{\alpha}=\mathcal{H}$, and $\rho_{0}\left(T_{w}\right)$ induces an operator on $\bar{M}_{0}^{\alpha}$. By our choice of $\alpha$, Corollary 4.8 implies that $\bar{M}_{0}^{\alpha}$ can be identified with $M^{\prime}$, the $\mathcal{H}_{n, r_{1}}$-module corresponding to $M$ for $\mathcal{H}$, and the action of $\widetilde{\mathcal{H}}_{\alpha}$ on $\bar{M}_{0}^{\alpha}$ coincides with the action of $\mathcal{H}_{n, r_{1}}$ on $M^{\prime}$. In particular, the action of $\rho_{0}\left(T_{w}\right)$ on $\bar{M}_{0}^{\alpha}$ corresponds to the action of $T_{w}$ on $M^{\prime}$ (we regard $\left.T_{w} \in \mathcal{H}_{n} \subset \mathcal{H}_{n, r_{1}}\right)$. Since $T_{w}\left(w \in \mathfrak{S}_{n}\right)$ are linearly independent as operators on $M^{\prime}$, we see that $\rho_{0}\left(T_{w}\right)$ are linearly independent as asserted.

By (6.1.5), we regard $\mathcal{H}_{n}$ as a subalgebra of $\overline{\mathcal{H}}^{\mathbf{p}}$, and define the elements $T_{1}, \ldots$, $T_{n-1} \in \overline{\mathcal{H}}^{\mathbf{p}}$ by the generators of $\mathcal{H}_{n}$.

6.2. We shall determine the commutation relations between $T_{j}$ and $\xi_{k}$. In view of Lemma 5.5, we compare the elements $\bar{m}_{\Omega} T_{j} \xi_{k}$ and $\bar{m}_{\Omega} \xi_{k} T_{j}$. First we compute the element $\bar{m}_{\Omega} T_{j}$ for $T_{j} \in \mathcal{H}_{n}$. Since $\bar{m}_{\Omega} T_{j}=\sum_{\omega \in \Omega} \bar{m}_{\omega} T_{d(\omega)} T_{j}$, we compute 
$m_{\omega} T_{d(\omega)} T_{j}$. Let $I=\coprod I_{k}$ be the partition corresponding to $\omega$. Assume that $j \in I_{k}$ and $j+1 \in I_{k^{\prime}}$. Then we see that

$$
T_{d(\omega)} T_{j}= \begin{cases}T_{d(\omega) s_{j}} & \text { if } k \leq k^{\prime}, \\ T_{d(\omega) s_{j}}+\left(q-q^{-1}\right) T_{d(\omega)} & \text { if } k>k^{\prime},\end{cases}
$$

where $s_{j}$ is the element in $\mathfrak{S}_{n}$ corresponding to $T_{j}$. Note that $m_{\omega}=u_{\mathbf{a}}^{+}=m_{\lambda}$, where $\lambda$ is the multi-partition obtained from $\omega$ by rearranging the rows. Put $\mathfrak{t}_{\omega}=$ $\mathfrak{t}^{\lambda} d(\omega) \in \operatorname{Std}(\lambda)$. Put $\mathfrak{v}_{\omega}=\mathfrak{t}_{\omega} s_{j}$. If $k \neq k^{\prime}$, then $\mathfrak{v}_{\omega} \in \operatorname{Std}(\lambda)$ and it is expressed as $\mathfrak{t}_{\omega^{\prime}}$, where $\omega^{\prime} \in \Omega$ is obtained from $\omega$ by exchanging $j$ and $j+1$ in $I_{k}$ and $I_{k^{\prime}}$. One can write $m_{\omega} T_{d(\omega)}=m_{S_{\omega} \mathfrak{t}_{\omega}}$ and $m_{\omega} T_{d(\omega) s_{j}}=m_{S_{\omega} \mathfrak{b}_{\omega}}$, where $S_{\omega}=\omega\left(\mathfrak{t}^{\lambda}\right) \in \mathcal{T}_{0}^{\mathbf{P}}(\lambda, \omega)$. Hence we have

$$
\bar{m}_{\omega} T_{d(\omega)} T_{j}= \begin{cases}\bar{m}_{S_{\omega} \mathfrak{v}_{\omega}} & \text { if } k=k^{\prime}, \\ \bar{m}_{S_{\omega} \mathfrak{t}_{\omega^{\prime}}} & \text { if } k<k^{\prime}, \\ \bar{m}_{S_{\omega} \mathfrak{t}_{\omega^{\prime}}}+\left(q-q^{-1}\right) \bar{m}_{S_{\omega} \mathfrak{t}_{\omega}} & \text { if } k>k^{\prime} .\end{cases}
$$

Note that in the first case, by [DJM, Proposition 3.18], $\bar{m}_{S_{\omega} \mathfrak{v}_{\omega}}$ is expressed as a linear combination of basis elements $\bar{m}_{S^{\prime} \mathfrak{v}}$ such that $\omega_{\mathfrak{v}}=\omega$. It follows from (6.2.1) that

$$
\begin{aligned}
\bar{m}_{\Omega} T_{j} & =\sum_{\substack{\omega \in \Omega \\
b_{\omega}(j)<b_{\omega}(j+1)}} \bar{m}_{S_{\omega \mathfrak{t}_{\omega^{\prime}}}} \\
& +\sum_{\substack{\omega \in \Omega \\
b_{\omega}(j)=b_{\omega}(j+1)}} \bar{m}_{S_{\omega^{\prime} \mathfrak{b}_{\omega}}}+\sum_{\substack{\omega \in \Omega \\
b_{\omega}(j)>b_{\omega}(j+1)}}\left(\bar{m}_{S_{\omega^{\prime} \boldsymbol{t}^{\prime}}}+\left(q-q^{-1}\right) \bar{m}_{S_{\omega} \mathfrak{t}_{\omega}}\right),
\end{aligned}
$$

where $\omega^{\prime} \in \Omega$ is obtained from $\omega$ by $s_{j}$ as above, and $\mathfrak{v}_{\omega}=\mathfrak{t}_{\omega} s_{j}$. Thus by (6.1.3) and (6.1.4), we have

$$
\begin{aligned}
\bar{m}_{\Omega} T_{j} \xi_{k} & =\sum_{\substack{\omega \in \Omega \\
b_{\omega}(j) \neq b_{\omega}(j+1)}} Q_{b_{\omega^{\prime}}(k)}^{\mathbf{p}} \bar{m}_{S_{\omega^{\prime} \omega^{\prime}}} \\
& +\sum_{\substack{\omega \in \Omega \\
b_{\omega}(j)=b_{\omega}(j+1)}} Q_{b_{\omega}(k)}^{\mathbf{p}} \bar{m}_{S_{\omega} \mathfrak{v}_{\omega}}+\sum_{\substack{\omega \in \Omega \\
b_{\omega}(j)>b_{\omega}(j+1)}} Q_{b_{\omega}(k)}^{\mathbf{p}}\left(q-q^{-1}\right) \bar{m}_{S_{\omega} \mathfrak{t}_{\omega}} .
\end{aligned}
$$

On the other hand, we have

$$
\begin{aligned}
\bar{m}_{\Omega} \xi_{k} T_{j} & =\sum_{\substack{\omega \in \Omega \\
b_{\omega}(j) \neq b_{\omega}(j+1)}} Q_{b_{\omega}(k)}^{\mathbf{p}} \bar{m}_{S_{\omega} \mathfrak{t}_{\omega^{\prime}}} \\
& +\sum_{\substack{\omega \in \Omega \\
b_{\omega}(j)=b_{\omega}(j+1)}} Q_{b_{\omega}(k)}^{\mathbf{p}} \bar{m}_{S_{\omega} \mathfrak{v}_{\omega}}+\sum_{\substack{\omega \in \Omega \\
b_{\omega}(j)>b_{\omega}(j+1)}} Q_{b_{\omega}(k)}^{\mathbf{p}}\left(q-q^{-1}\right) \bar{m}_{S_{\omega} \mathfrak{t}_{\omega}} .
\end{aligned}
$$

It follows that

$$
\bar{m}_{\Omega}\left(T_{j} \xi_{k}-\xi_{k} T_{j}\right)=\sum_{\substack{\omega \in \Omega \\ b_{\omega}(j) \neq b_{\omega}(j+1)}}\left(Q_{b_{\omega^{\prime}}(k)}^{\mathbf{p}}-Q_{b_{\omega}(k)}^{\mathbf{p}}\right) \bar{m}_{S_{\omega^{\prime} t_{\omega^{\prime}}}} .
$$

Note that if $k \neq j, j+1$, then $b_{\omega}(k)=b_{\omega^{\prime}}(k)$ for any $\omega$. It follows that

$$
T_{j} \xi_{k}=\xi_{k} T_{j} \text { if } k \neq j, j+1 \text {. }
$$


6.3. Let $A$ be a square matrix of degree $g$ whose $i j$-entry is given by $\left(Q_{j}^{\mathbf{p}}\right)^{i-1}$ for $1 \leq i, j \leq g$. Thus $A$ is the Vandermonde matrix, and $\Delta=\operatorname{det} A=\prod_{i>j}\left(Q_{i}^{\mathbf{p}}-Q_{j}^{\mathbf{p}}\right)$. We pose the following assumption so that $\Delta^{-1} \in R$.

$$
Q_{i}^{\mathbf{p}}-Q_{j}^{\mathbf{p}} \text { are units in } R \text { for any } i \neq j \text {. }
$$

We express $A^{-1}=\Delta^{-1} B$ with $B=\left(h_{i j}\right)$ for $h_{i j} \in R$. We define a polynomial $F_{i}(X) \in R[X]$, for $1 \leq i \leq g$, by

$$
F_{i}(X)=\sum_{j=1}^{g} h_{i j} X^{j-1}
$$

We denote by $\Omega_{j}^{[c]}$ the set of $\omega \in \Omega$ such that $b_{\omega}(j)=c$ for $1 \leq j \leq n, 1 \leq c \leq g$. As in 6.2 , one can write $\bar{m}_{\Omega}=\sum_{\omega \in \Omega} \bar{m}_{S_{\omega} \mathrm{t}_{\omega}}$, and so

$$
\bar{m}_{\Omega} \xi_{j}^{b}=\sum_{\omega \in \Omega}\left(Q_{b_{\omega}(j)}^{\mathbf{p}}\right)^{b} \bar{m}_{S_{\omega} \boldsymbol{t}_{\omega}}=\sum_{c=1}^{g}\left(Q_{c}^{\mathbf{p}}\right)^{b} \sum_{\omega \in \Omega_{j}^{[c]}} \bar{m}_{S_{\omega} \boldsymbol{t}_{\omega}}
$$

for $b=0, \ldots, g-1$. We regard (6.3.2) as a system of linear equations with unknown variables $\sum_{\omega \in \Omega_{j}^{[c]}} \bar{m}_{S_{\omega} t_{\omega}}$. Since $\Delta^{-1} \in R$, we see that

$$
\sum_{\omega \in \Omega_{j}^{[c]}} \bar{m}_{S_{\omega} \mathfrak{t}_{\omega}}=\bar{m}_{\Omega} \cdot \Delta^{-1} \sum_{b=1}^{g} h_{c b} \xi_{j}^{b-1}=\bar{m}_{\Omega} \cdot \Delta^{-1} F_{c}\left(\xi_{j}\right) .
$$

Repeating a similar procedure, we have

$$
\sum_{\omega \in \Omega_{j}^{\left[c_{1}\right]} \cap \Omega_{j+1}^{\left[c_{2}\right]}} \bar{m}_{S_{\omega} t_{\omega}}=\bar{m}_{\Omega} \cdot \Delta^{-2} F_{c_{1}}\left(\xi_{j}\right) F_{c_{2}}\left(\xi_{j+1}\right) .
$$

By applying $T_{j}$ on both sides of (6.3.3), and by using (6.2.1), we have

$$
\sum_{\omega \in \Omega_{j}^{\left[c_{1}\right]} \cap \Omega_{j+1}^{\left[c_{2}\right]}} \bar{m}_{S_{\omega} \boldsymbol{t}_{\omega^{\prime}}}= \begin{cases}\bar{m}_{\Omega} \cdot \Delta^{-2} F_{c_{1}}\left(\xi_{j}\right) F_{c_{2}}\left(\xi_{j+1}\right) T_{j} & \text { if } c_{1}<c_{2}, \\ \bar{m}_{\Omega} \cdot \Delta^{-2} F_{c_{1}}\left(\xi_{j}\right) F_{c_{2}}\left(\xi_{j+1}\right)\left(T_{j}-\left(q-q^{-1}\right)\right) & \text { if } c_{1}>c_{2} .\end{cases}
$$

We show the following lemma, which is analogous to [Sh, Lemma 3.4].

Lemma 6.4. For $j=1, \ldots, n-1$, we have

$$
\begin{aligned}
T_{j} \xi_{j+1} & =\xi_{j} T_{j}+\Delta^{-2} \sum_{c_{1}>c_{2}}\left(Q_{c_{2}}^{\mathbf{p}}-Q_{c_{1}}^{\mathbf{p}}\right)\left(q-q^{-1}\right) F_{c_{1}}\left(\xi_{j}\right) F_{c_{2}}\left(\xi_{j+1}\right), \\
T_{j} \xi_{j} & =\xi_{j+1} T_{j}-\Delta^{-2} \sum_{c_{1}>c_{2}}\left(Q_{c_{2}}^{\mathbf{p}}-Q_{c_{1}}^{\mathbf{p}}\right)\left(q-q^{-1}\right) F_{c_{1}}\left(\xi_{j}\right) F_{c_{2}}\left(\xi_{j+1}\right), \\
T_{j} \xi_{k} & =\xi_{k} T_{j} \quad(k \neq j, j+1) .
\end{aligned}
$$

Proof. The third formula is already shown in (6.2.3). So assume that $k=j$ or $j+1$. Substituting (6.3.4) into (6.2.2), and by using Lemma 5.5, we have

$$
\begin{aligned}
T_{j} \xi_{k}-\xi_{k} T_{j}= & \varepsilon \Delta^{-2}\left\{\sum_{c_{1}<c_{2}}\left(Q_{c_{2}}^{\mathbf{p}}-Q_{c_{1}}^{\mathbf{p}}\right) F_{c_{1}}\left(\xi_{j}\right) F_{c_{2}}\left(\xi_{j+1}\right) T_{j}\right. \\
& \left.+\sum_{c_{1}>c_{2}}\left(Q_{c_{2}}^{\mathbf{p}}-Q_{c_{1}}^{\mathbf{p}}\right) F_{c_{1}}\left(\xi_{j}\right) F_{c_{2}}\left(\xi_{j+1}\right)\left(T_{j}-\left(q-q^{-1}\right)\right)\right\},
\end{aligned}
$$


where $\varepsilon=1$ (resp. $\varepsilon=-1$ ) if $k=j$ (resp. $k=j+1$ ).

We note that the following formula holds.

$$
\xi_{j+1}-\xi_{j}=\Delta^{-2} \sum_{c_{1}<c_{2}}\left(Q_{c_{2}}^{\mathbf{p}}-Q_{c_{1}}^{\mathbf{p}}\right)\left\{F_{c_{1}}\left(\xi_{j}\right) F_{c_{2}}\left(\xi_{j+1}\right)-F_{c_{2}}\left(\xi_{j}\right) F_{c_{1}}\left(\xi_{j+1}\right)\right\}
$$

In fact, it is enough to compare the values at $\bar{m}_{S_{\omega} \boldsymbol{t}_{\omega}} \in \bar{M}^{\Omega}$. This is essentially the same as the case where $\mathbf{p}=\left(1^{r}\right)$, and in that case the formula is proved in $\mathbf{S h}$, (3.4.2)].

Now (6.4.1) can be written, by making use of (6.4.2), as

$$
\begin{aligned}
T_{j} \xi_{k}-\xi_{k} T_{j}= & \varepsilon\left(\xi_{j+1}-\xi_{j}\right) T_{j} \\
& -\varepsilon \sum_{c_{1}>c 2}\left(Q_{c_{2}}^{\mathbf{p}}-Q_{c_{1}}^{\mathbf{p}}\right)\left(q-q^{-1}\right) F_{c_{1}}\left(\xi_{j}\right) F_{c_{2}}\left(\xi_{j+1}\right) .
\end{aligned}
$$

The first and the second equalities in the lemma follow from this.

6.5. For each $\alpha \in \Delta_{n, g}$ and for $k=1, \ldots, g$, we define $T_{\alpha, 0}^{[k]} \in \overline{\mathcal{H}}^{\mathbf{p}}$ as follows. We regard $T_{0}^{[k]} \in \mathcal{H}_{n_{k}, r_{k}}$ as an element in $\mathcal{H}_{n_{1}, r_{1}} \otimes \cdots \otimes \mathcal{H}_{n_{g}, r_{g}}$, and we denote by $T_{\alpha, 0}^{[k]} \in \overline{\mathcal{H}}_{\alpha}^{\mathbf{p}}$ the diagonal matrix consisting of $T_{0}^{[k]}$ in the diagonal entries under the isomorphism in Proposition 5.3. In particular, one can write

$$
\bar{m}_{\Omega} T_{\alpha, 0}^{[k]}=\sum_{\omega \in \Omega^{\alpha}} \bar{m}_{\omega} T_{d(\omega)} L_{a_{k}+1},
$$

where $\Omega^{\alpha}=\left\{\omega \in \Omega \mid \alpha_{\mathbf{p}}(\omega)=\alpha\right\}$. Thus we see that $T_{\alpha, 0}^{[k]}$ acts on $\bar{M}^{\Omega, \alpha}=\bar{M}^{\Omega} \cap \bar{M}^{\alpha}$ as $L_{a_{k}+1}$, and annihilates $\bar{M}^{\Omega, \alpha^{\prime}}$ for any $\alpha^{\prime} \neq \alpha$.

For a given $\omega \in \Omega$, put $c_{i}=b_{\omega}(i)$ for $i=1, \ldots, n$. We define $F_{\omega}(\xi) \in \overline{\mathcal{H}}^{\mathbf{p}}$ by

$$
F_{\omega}(\xi)=F_{c_{1}}\left(\xi_{1}\right) F_{c_{2}}\left(\xi_{2}\right) \cdots F_{c_{n}}\left(\xi_{n}\right) .
$$

We have the following lemma.

Lemma 6.6. Under the assumption of (6.3.1), the elements

$$
\xi_{i}(1 \leq i \leq n), \quad T_{j}(1 \leq j \leq n-1), \quad T_{\alpha, 0}^{[k]}\left(\alpha \in \Delta_{n, g}, 1 \leq k \leq g\right)
$$

generate $\overline{\mathcal{H}}^{\mathbf{p}}$.

Proof. Let $\mathcal{K}$ be the subalgebra of $\overline{\mathcal{H}}^{\mathbf{p}}$ generated by elements in the lemma. In view of Lemma 5.5 , it is enough to show that $\bar{M}^{\Omega}=\bar{m}_{\Omega} \mathcal{K}$. First we show that

$$
\bar{m}_{\omega} \in \bar{m}_{\Omega} \mathcal{K}
$$

for any $\omega \in \Omega$. In fact, we have $\bigcap_{i=1}^{n} \Omega_{i}^{\left[c_{i}\right]}=\{\omega\}$ with $c_{i}=b_{\omega(i)}$. Hence by repeating the argument used to prove (6.3.3), we see that

$$
\bar{m}_{\omega} T_{d(\omega)}=\bar{m}_{S_{\omega} \mathfrak{t}_{w}}=\bar{m}_{\Omega} \cdot \Delta^{-n} F_{\omega}(\xi) .
$$

This implies that $\bar{m}_{\omega} T_{d(\omega)} \in \bar{m}_{\Omega} \mathcal{K}$. Since $T_{d(\omega)}$ is an invertible element in $\mathcal{K}$, we obtain (6.6.1). 
Now take $\bar{m}_{\omega}$ and put $\alpha=\alpha_{\mathbf{p}}(\omega)$. We know that $\bar{m}_{\omega} \in \bar{M}_{0}^{\omega}$, and that $\bar{M}_{0}^{\omega}=$ $\bar{m}_{\omega} \widetilde{\mathcal{H}}_{\alpha}$ (see the proof of Lemma 4.10). Note that $\widetilde{H}_{\alpha}$ is generated by $L_{a_{k}+1}$ and $\widetilde{H}_{\alpha} \cap \mathcal{H}_{n}$, and the action of $L_{a_{k}+1}$ on $\bar{M}_{0}^{\alpha}$ coincides with that of $T_{\alpha, 0}^{[k]}$. It follows that $\bar{M}_{0}^{\omega}=\bar{m}_{\omega} \widetilde{\mathcal{H}}_{\alpha} \subset \bar{m}_{\Omega} \mathcal{K}$. Here $\bar{M}_{0}^{\omega}$ has the basis $\left\{\bar{m}_{S \mathfrak{t}}\right\}$ with $S \in \mathcal{T}_{0}^{\mathbf{p}}(\lambda, \omega)$ and $\mathfrak{t} \in \operatorname{Std}(\lambda)_{0}$. While the basis of $\bar{M}^{\omega}$ is given by $\left\{\bar{m}_{S \mathfrak{t}^{\prime}}\right\}$ for $S \in \mathcal{T}^{\mathbf{p}}(\lambda, \omega)$ and $\mathfrak{t}^{\prime} \in \operatorname{Std}(\lambda)$. If we take $\mathfrak{t}=\mathfrak{t}^{\lambda} \in \operatorname{Std}(\lambda)_{0}$, any $\mathfrak{t}^{\prime}$ is obtained as $\mathfrak{t}^{\prime}=\mathfrak{t} d\left(\mathfrak{t}^{\prime}\right)$, and we have $\bar{m}_{S \mathrm{t}^{\prime}}=\bar{m}_{S \mathrm{t}} T_{d\left(\mathfrak{t}^{\prime}\right)}$. It follows that $\bar{M}^{\omega} \subseteq \bar{M}_{0}^{\omega} \mathcal{H}_{n} \subseteq \bar{m}_{\Omega} \mathcal{K}$ for any $\omega \in \Omega$, and so $\bar{M}^{\Omega}=\bar{m}_{\Omega} \mathcal{K}$. The lemma is proved.

6.7. Recall that $\overline{\mathcal{H}}^{\mathbf{p}}=\bigoplus_{\alpha \in \Delta_{n, g}} \overline{\mathcal{H}}_{\alpha}^{\mathbf{p}}$. For each $\alpha \in \Delta_{n, g}$, we denote by $T_{\alpha, j}$ the projection of $T_{j}$ onto $\overline{\mathcal{H}}_{\alpha}^{\mathbf{p}}$. Also we denote by $\xi_{\alpha, i}$ the projection of $\xi_{i}$ onto $\overline{\mathcal{H}}_{\alpha}^{\mathbf{p}}$. Hence we have $T_{j}=\sum_{\alpha} T_{\alpha, j}$ and $\xi_{i}=\sum_{\alpha} \xi_{\alpha, i}$. It follows from the construction that under the isomorphism $\bar{M}_{0}^{\omega} \simeq M^{\omega^{[1]}} \otimes \cdots \otimes M^{\omega^{[g]}}$, the action of $T_{\alpha, a_{k}+i}$ corresponds to the action of $T_{i}^{[k]}$ on $M^{\omega^{[k]}}$.

We note the relation

$$
\xi_{\alpha, i} T_{\alpha, 0}^{[k]}=T_{\alpha, 0}^{[k]} \xi_{\alpha, i}
$$

for any $i$ and any $k$. In fact, by (5.3.1), it is enough to show the formula regarding $\xi_{\alpha, i}$ and $T_{\alpha, 0}^{[k]}$ as operators on $\bar{M}_{0}^{\Omega, \alpha}$. Under the isomorphism $\bar{M}_{0}^{\omega} \simeq M^{\omega^{[1]}} \otimes \cdots \otimes$ $M^{\omega^{[g]}}$ for $\omega \in \Omega$ such that $\alpha_{\mathbf{p}}(\omega)=\alpha, \xi_{\alpha, a_{h}+i}$ corresponds to the operator $\xi_{i}^{[h]}$ on $M^{\omega^{[h]}}$, where $\xi_{i}^{[h]}$ is an element of $\mathcal{H}_{n_{h}, r_{h}}$ defined similar to $\xi_{i}$ for $\overline{\mathcal{H}}^{\mathbf{p}}$ (i.e., the special case where $n=n_{h}, r=r_{h}, g=1, \mathbf{p}=\left(r_{h}\right)$ ). But it is easy to see that in this case $\xi_{i}^{[h]}$ is a scalar multiplication on $M^{\omega^{[h]}}$ by $Q_{h}^{\mathbf{p}}$. Hence $\xi_{\alpha, i}$ is a scalars operator on $\bar{M}^{\omega}$, and so commutes with $T_{\alpha, 0}^{[k]}$. Equation (6.7.1) follows from this.

For each $\omega \in \Omega$ and $\alpha \in \Delta_{n, g}$, let

$$
F_{\omega}\left(\xi_{\alpha}\right)=F_{c_{1}}\left(\xi_{\alpha, 1}\right) F_{c_{2}}\left(\xi_{\alpha, 2}\right) \cdots F_{c_{n}}\left(\xi_{\alpha, n}\right)
$$

with $c_{i}=b_{\omega}(i)$. We claim that

$$
F_{\omega}\left(\xi_{\alpha}\right)=0 \quad \text { unless } \alpha_{\mathbf{p}}(\omega)=\alpha \text {. }
$$

In fact, we have $\bar{m}_{\Omega} F_{\omega}\left(\xi_{\alpha}\right) \in \bar{M}^{\alpha^{\prime}}$ by (6.6.2), where $\alpha^{\prime}=\alpha_{\mathbf{p}}(\omega)$. But since $F_{\omega}\left(\xi_{\alpha}\right) \in \overline{\mathcal{H}}_{\alpha}^{\mathbf{p}}=\varphi_{\Omega, \alpha} \overline{\mathcal{S}}_{\alpha}^{\mathbf{p}} \varphi_{\Omega, \alpha}$, we have $\bar{m}_{\Omega} F_{\omega}\left(\xi_{\alpha}\right) \in \bar{M}^{\alpha}$. It follows that $\bar{m}_{\Omega} F_{\omega}\left(\xi_{\alpha}\right)$ $=0$ unless $\alpha_{\mathbf{p}}(\omega)=\alpha$, and the claim follows.

The following theorem gives a presentation of $\overline{\mathcal{H}}^{\text {p }}$.

Theorem 6.8. Assume that (6.3.1) holds. Recall that $Q_{k}^{\mathbf{p}}=Q_{p_{k}+r_{k}}$. Then for each $\alpha \in \Delta_{n, g}$, the algebra $\overline{\mathcal{H}}_{\alpha}^{\mathbf{p}}$ is generated by

$$
\xi_{\alpha, i}(1 \leq i \leq n), \quad T_{\alpha, j}(1 \leq j \leq n-1), \quad T_{\alpha, 0}^{[k]}(1 \leq k \leq g)
$$


with the relations

$$
\begin{aligned}
& \left(T_{\alpha, i}-q\right)\left(T_{\alpha, i}+q^{-1}\right)=0 \quad(1 \leq i \leq n-1) \\
& T_{\alpha, i} T_{\alpha, i+1} T_{\alpha, i}=T_{\alpha, i+1} T_{\alpha, i} T_{\alpha, i+1} \quad(1 \leq i \leq n-2), \\
& T_{\alpha, i} T_{\alpha, j}=T_{\alpha, j} T_{\alpha, i} \quad(1 \leq i, j \leq n-1,|i-j| \geq 2), \\
& \left(T_{\alpha, 0}^{[k]}-Q_{p_{k}+1}\right) \cdots\left(T_{\alpha, 0}^{[k]}-Q_{p_{k}+r_{k}}\right)=0 \quad(1 \leq k \leq g), \\
& T_{\alpha, 0}^{[k]} T_{\alpha, a_{k}+1} T_{\alpha, 0}^{[k]} T_{\alpha, a_{k}+1}=T_{\alpha, a_{k}+1} T_{\alpha, 0}^{[k]} T_{\alpha, a_{k}+1} T_{\alpha, 0}^{[k]} \quad(1 \leq k \leq g), \\
& T_{\alpha, 0}^{[k]}=T_{\alpha, a_{k}} \cdots T_{\alpha, a_{k-1}+2} T_{\alpha, a_{k-1}+1} T_{\alpha, 0}^{[k-1]} T_{\alpha, a_{k-1}+1} T_{\alpha, a_{k-1}+2} \cdots T_{\alpha, a_{k}}, \\
& T_{\alpha, 0}^{[k]} T_{\alpha, j}=T_{\alpha, j} T_{\alpha, 0}^{[k]} \quad\left(j \neq a_{k}, a_{k}+1\right), \\
& \left(\xi_{\alpha, i}-Q_{1}^{\mathbf{p}}\right)\left(\xi_{\alpha, i}-Q_{2}^{\mathbf{p}}\right) \cdots\left(\xi_{\alpha, i}-Q_{g}^{\mathbf{p}}\right)=0 \quad(1 \leq i \leq n), \\
& \xi_{\alpha, i} \xi_{\alpha, j}=\xi_{\alpha, j} \xi_{\alpha, i} \quad(1 \leq i, j \leq n), \\
& F_{\omega}\left(\xi_{\alpha}\right)=0 \quad i f \alpha_{\mathbf{p}}(\omega) \neq \alpha, \\
& T_{\alpha, j} \xi_{\alpha, j+1}=\xi_{\alpha, j} T_{\alpha, j} \quad \Delta_{c_{1}<c_{2}} \\
& \quad+\Delta_{c_{2}}^{-2} \sum_{c_{1}}\left(Q^{\mathbf{p}}-Q^{\mathbf{p}}\right)\left(q-q_{c_{1}}\left(\xi_{\alpha, j}\right) F_{c_{2}}\left(\xi_{\alpha, j+1}\right),\right. \\
& T_{\alpha, j} \xi_{\alpha, j}=\xi_{\alpha, j+1} T_{\alpha, j} \\
& \quad-\Delta^{-2} \sum_{c_{1}<c_{2}}\left(Q_{c_{2}}^{\mathbf{p}}-Q_{c_{1}}^{\mathbf{p}}\right)\left(q-q^{-1}\right) F_{c_{1}}\left(\xi_{\alpha, j}\right) F_{c_{2}}\left(\xi_{\alpha, j+1}\right), \\
& T_{\alpha, j} \xi_{\alpha, k}=\xi_{\alpha, k} T_{\alpha, j} \quad(k \neq j, j+1), \\
& T_{\alpha, 0}^{[k]} \xi_{i}=\xi_{i} T_{\alpha, 0}^{[k]} \quad(1 \leq i \leq n, 1 \leq k \leq g) .
\end{aligned}
$$

Proof. One sees that these elements generate $\overline{\mathcal{H}}_{\alpha}^{\mathbf{p}}$ by Lemma 6.6. We show that these generators satisfy the relations (A1)-(A14). (A1)-(A3) follow from the relations for $\mathcal{H}_{n}$. Relation (A8) follows from (6.1.2). Relation (A9) is also clear from 6.1. Relation (A10) follows from (6.7.2). (A11)-(A13) follows from Lemma 6.4. Relation (A14) is given in (6.7.1). We show the remaining relations (A4)-(A7). We may prove the formulas, by regarding $T_{\alpha, 0}^{[k]}$ and $T_{\alpha, j}$ as operators on $\bar{M}_{0}^{\omega}$ for $\omega \in \Omega$ such that $\alpha_{\mathbf{p}}(\omega)=\alpha$ by (5.3.1). Since $T_{\alpha, 0}^{[k]}$ corresponds to the action of $T_{0}^{[k]} \in \mathcal{H}_{n_{k}, r_{k}}$ on $M^{\omega^{[k]}}$, and $T_{\alpha, a_{k}+i}$ corresponds to the action of $T_{i}^{[k]},(\mathrm{A} 4)$, (A5) and (A7) follow from the relations for $\mathcal{H}_{n_{k}, r_{k}}$. While (A6) follows from the property that $T_{\alpha, 0}^{[k]}$ is the restriction of $L_{a_{k}+1}$ on $\bar{M}^{\Omega, \alpha}$. Thus those generators satisfy the relations (A1)-(A14).

Next we show that (A1)-(A14) gives a fundamental relation for $\overline{\mathcal{H}}_{\alpha}^{\mathbf{p}}$. Let $\widehat{\mathcal{H}}_{\alpha}$ be the algebra with generators $\widehat{\xi}_{\alpha, i}, \widehat{T}_{\alpha, j}$ and $\widehat{T}_{\alpha, 0}^{[k]}$, and relations as in the theorem. (We denote by $\widehat{X}$ the generator in $\widehat{\mathcal{H}}_{\alpha}$ corresponding to the generator $X$ in $\overline{\mathcal{H}}_{\alpha}^{\mathbf{p}}$.) Let $\widehat{\mathcal{H}}_{\alpha}^{0}$ be the subalgebra of $\widehat{\mathcal{H}}_{\alpha}$ generated by $\widehat{T}_{\alpha, i}^{[k]}$ for $k=1, \ldots, g, i=0, \ldots, n_{k}-1$. Recall that $\widehat{T}_{\alpha, i}^{[k]}=\widehat{T}_{\alpha, a_{k}+i}$ for $1 \leq i \leq n_{k}-1$. Then by the relations in the theorem, $\widehat{\mathcal{H}}_{\alpha}^{0}$ is isomorphic to the quotient of the algebra $\mathcal{H}_{n_{1}, r_{1}} \otimes \cdots \otimes \mathcal{H}_{n_{g}, r_{g}}$. Also, we note that the subalgebra $\widehat{\mathcal{H}}_{n}$ of $\widehat{\mathcal{H}}_{\alpha}$ generated by $\widehat{T}_{\alpha, j}$ is the quotient of $\mathcal{H}_{n}$. We denote by $\widehat{T}_{\alpha, w}$ the image of $T_{w} \in \mathcal{H}_{n}$ to $\widehat{\mathcal{H}}_{n}$ for $w \in \mathfrak{S}_{n}$. Let $\mathfrak{S}_{\alpha}$ be the Young subgroup of $\mathfrak{S}_{n}$ corresponding to the composition $\alpha$ of $n$. Let $\widehat{\Xi}_{\alpha}$ be the subalgebra of $\widehat{\mathcal{H}}_{\alpha}$ 
generated by $\widehat{\xi}_{\alpha, 1}, \ldots, \widehat{\xi}_{\alpha, n}$. For each $\omega \in \Omega^{\alpha}$, we define $F_{\omega}\left(\widehat{\xi}_{\alpha}\right) \in \widehat{\Xi}_{\alpha}$ in a similar way as $F_{\omega}\left(\xi_{\alpha}\right)$, but replacing $\xi_{\alpha, i}$ by $\widehat{\xi}_{\alpha, i}$. We show that

(6.8.1) Any element of $\widehat{\mathcal{H}}_{\alpha}$ can be written as a linear combination of elements in

$$
\mathcal{C}=\left\{F_{\omega}\left(\widehat{\xi}_{\alpha}\right) \widehat{\mathcal{H}}_{\alpha}^{0} \widehat{T}_{\alpha, w} \mid \omega \in \Omega^{\alpha}, w \in \mathfrak{S}_{\alpha} \backslash \mathfrak{S}_{n}\right\}
$$

In fact, let $\widehat{\mathcal{H}}_{\alpha}^{\natural}$ be the subalgebra of $\widehat{\mathcal{H}}_{\alpha}$ generated by $\widehat{T}_{\alpha, j}$ and $\widehat{T}_{\alpha, 0}^{[k]}$. Then by the commuting relations in the theorem, $\widehat{\mathcal{H}}_{\alpha}$ can be written as

$$
\widehat{\mathcal{H}}_{\alpha}=\sum_{c_{1}, \ldots, c_{n}} \widehat{\xi}_{\alpha, 1}^{c_{1}} \widehat{\xi}_{\alpha, 2}^{c_{2}} \cdots \widehat{\xi}_{\alpha, n}^{c_{n}} \widehat{\mathcal{H}}_{\alpha}^{\natural},
$$

where $c_{i}$ are integers such that $0 \leq c_{i} \leq g-1$. It is easy to see that any element in $\widehat{\Xi}_{\alpha}$ can be written as a linear combination of $F_{\omega}\left(\widehat{\xi}_{\alpha}\right)$ for various $\omega \in \Omega$. Thus by $(\mathrm{A} 10)$, any element in $\widehat{\mathcal{H}}_{\alpha}$ is written as a linear combination of $F_{\omega}\left(\widehat{\xi}_{\alpha}\right) \widehat{\mathcal{H}}_{\alpha}^{\natural}$ with $\omega \in \Omega^{\alpha}$. We now concentrate on $\widehat{\mathcal{H}}_{\alpha}^{\natural}$. Define $\widehat{L}_{i}^{[k]} \in \widehat{\mathcal{H}}_{\alpha}^{0}$ by

$$
\widehat{L}_{i}^{[k]}=\widehat{T}_{\alpha, a_{k}+i-1} \cdots \widehat{T}_{\alpha, a_{k}+2} \widehat{T}_{\alpha, a_{k}+1} \widehat{T}_{\alpha, 0}^{[k]} \widehat{T}_{\alpha, a_{k}+1} \widehat{T}_{\alpha, a_{k}+2} \cdots \widehat{T}_{\alpha, a_{k}+i-1}
$$

for $i=1, \ldots, n_{k}$. Then $L_{i}^{[k]}$ commutes with $\widehat{T}_{\alpha, j}$ for $j \neq a_{k}+i-1, a_{k}+i$ and we have

$$
\widehat{T}_{\alpha, a_{k}+i} \widehat{L}_{i}^{[k]} \widehat{T}_{\alpha, a_{k}+i}= \begin{cases}\widehat{L}_{i+1}^{[k]} & \text { if } i \neq n_{k}, \\ \widehat{L}_{1}^{[k+1]} & \text { if } i=n_{k} .\end{cases}
$$

by (A6). (Note that in the latter case, $\widehat{T}_{\alpha, a_{k}+n_{k}} \notin \widehat{\mathcal{H}}_{\alpha}^{0}$.) It follows that any element in $\widehat{\mathcal{H}}_{\alpha}^{\natural}$ can be written as a linear combination of the elements in $\widehat{L} \widehat{\mathcal{H}}_{n}$, where $\widehat{L}$ is the subalgebra of $\widehat{\mathcal{H}}_{\alpha}^{0}$ generated by $\widehat{L}_{i}^{[k]}$. Let $\mathcal{H}_{n, \alpha}$ be the subalgebra of $\mathcal{H}_{n}$ corresponding to the Young subgroup $\mathfrak{S}_{\alpha}$ of $\mathfrak{S}_{n}$, and let $\widehat{\mathcal{H}}_{n, \alpha}$ be the corresponding subalgebra of $\widehat{\mathcal{H}}_{n}$. Since $\widehat{\mathcal{H}}_{n}$ is the quotient of $\mathcal{H}_{n}$, it is written as a sum of $\widehat{\mathcal{H}}_{n, \alpha} \widehat{T}_{\alpha, w}$ with $w \in \mathfrak{S}_{\alpha} \backslash \mathfrak{S}_{n}$. Since $\widehat{\mathcal{H}}_{n, \alpha} \subset \widehat{\mathcal{H}}_{\alpha}^{0}$, one sees that $\widehat{\mathcal{H}}_{\alpha}^{\natural}=\sum_{w \in \mathfrak{S}_{\alpha} \backslash \mathfrak{S}_{n}} \widehat{\mathcal{H}}_{\alpha}^{0} \widehat{T}_{w}$. Hence (6.8.1) holds.

Since $\overline{\mathcal{H}}_{\alpha}^{\mathbf{p}}$ satisfies the same relations, we have a surjective homomorphism $\psi$ : $\widehat{\mathcal{H}}_{\alpha} \rightarrow \overline{\mathcal{H}}_{\alpha}^{\mathbf{p}}$. In order to show that $\psi$ is injective, it is enough to see that the set of elements in (6.8.1) gives an $R$-free basis of $\widehat{\mathcal{H}}_{\alpha}$ and that the image under $\psi$ of this basis gives a basis of $\overline{\mathcal{H}}_{\alpha}^{\mathbf{p}}$. We denote by $\mathcal{C}^{\prime}$ the image of $\mathcal{C}$ under $\psi$. By a similar argument as above, we see that $\mathcal{C}^{\prime}$ spans $\overline{\mathcal{H}}_{\alpha}^{\mathbf{p}}$ as an $R$-module. We show that $\mathcal{C}^{\prime}$ gives an $R$-free basis of $\overline{\mathcal{H}}^{\mathbf{p}}$. For this, it is enough to see that the elements in $\mathcal{C}^{\prime}$ are linearly independent over $R$, or equivalently, they are linearly independent over $K$, where $K$ is the quotient field of $R$. It is easy to see that the cardinality of the set $\mathcal{C}^{\prime}$ is equal to

$$
\left|\Omega^{\alpha}\right| \times \operatorname{dim} \mathcal{H}_{\alpha} \times n_{\alpha}=n_{\alpha}^{2} \times \operatorname{dim} \mathcal{H}_{\alpha}=\operatorname{dim} \overline{\mathcal{H}}_{\alpha}^{\mathbf{p}}
$$

by Proposition 5.3. Hence the elements of $\mathcal{C}^{\prime}$ are linearly independent, and $\mathcal{C}^{\prime}$ gives an $R$-free basis of $\overline{\mathcal{H}}_{\alpha}^{\mathbf{p}}$. This shows that the elements in $\mathcal{C}$ are also linearly independent, and so $\mathcal{C}$ is an $R$-free basis of $\widehat{\mathcal{H}}_{\alpha}$. Therefore $\psi$ is an isomorphism, and the theorem is proved.

Remark 6.9. In the case where $\mathbf{p}=(r), \overline{\mathcal{H}}_{\alpha}^{\mathbf{p}}=\overline{\mathcal{H}}^{\mathbf{p}}$ coincides with $\mathcal{H}$, and the fundamental relation $(\mathrm{A} 1)-(\mathrm{A} 14)$ is reduced to the fundamental relation for $\mathcal{H}$. On the other hand, in the case where $\mathbf{p}=\left(1^{r}\right), \mathcal{H}_{\alpha}$ is a subalgebra of $\mathcal{H}_{n}$ for each 
$\alpha \in \Delta_{n, r}$. Then $T_{\alpha, 0}^{[k]}$ turns out to be scalar operators, and the relations (A4)(A7), (A14) can be ignored. The remaining relations give the fundamental relation for $\overline{\mathcal{H}}_{\alpha}^{\mathbf{p}}$. Note that a similar argument as in the proof shows that the relations (A1)-(A3), (A8), (A9), (A11)-(A13) gives a fundamental relation for $\overline{\mathcal{H}}^{\mathbf{p}}$, which is nothing but the fundamental relation for the modified Ariki-Koike algebra given in Saws.

\section{Schur-Weyl DUALity}

7.1. It is known by [M] $\S 5]$ that the Schur-Weyl duality i.e., the double centralizer property holds between $\mathcal{H}$ and $\mathcal{S}=\operatorname{End}_{\mathcal{H}} M$. A similar duality also holds by SawS, Theorem 8.2] for the modified Ariki-Koike algebra $\overline{\mathcal{H}}$ on the action of the tensor space $V^{\otimes n}$. In our setting, $\overline{\mathcal{H}}$ coincides with $\overline{\mathcal{H}}^{\mathbf{p}}$ with $\mathbf{p}=\left(1^{r}\right)$, and $V^{\otimes n} \simeq \bar{M}$ as $\overline{\mathcal{H}} \mathbf{p}$-modules. In what follows we shall give a generalization of this property for the arbitrary $\mathbf{p}$, i.e., we show the Schur-Weyl duality between $\overline{\mathcal{S}}^{\mathbf{p}}$ and $\overline{\mathcal{H}} \mathbf{p}$ acting on $\bar{M}$. Although the proof is carried out for the action on $\bar{M}$, we formulate the theorem for $\overline{\mathcal{H}}^{\mathbf{p}}$-module $M_{\mathbf{p}}=\bigoplus M_{\mathbf{p}}^{\mu}$ which is isomorphic to $\bar{M}$, where $M_{\mathbf{p}}^{\mu}$ is a right ideal of $\overline{\mathcal{H}}^{\mathbf{p}}$, so that it fits to the situation above.

7.2. In order to give an expression of $\bar{M}^{\mu}$ as a right ideal of $\overline{\mathcal{H}}^{\mathbf{p}}$, we describe the cellular basis $m_{\mathfrak{s t}}^{\mathbf{p}}$ of $\overline{\mathcal{H}}^{\mathbf{p}}$ more explicitly. For each $\alpha=\left(n_{1}, \ldots, n_{g}\right) \in \Delta_{n, g}$ we define $F_{\alpha} \in \overline{\mathcal{H}}^{\mathbf{p}}$ by

$$
F_{\alpha}=\Delta^{-n} F_{c_{1}}\left(\xi_{1}\right) \cdots F_{c_{n}}\left(\xi_{n}\right),
$$

where

$$
\left(c_{1}, \ldots, c_{n}\right)=(\underbrace{1, \ldots, 1}_{n_{1} \text {-times }}, \underbrace{2, \ldots, 2}_{n_{2} \text {-times }}, \ldots, \underbrace{g, \ldots, g}_{n_{g} \text {-times }}) .
$$

If we define $\omega=\omega_{\alpha}$ as the unique element in $\Omega^{\alpha}$ such that $d(\omega)=1$, we see that $F_{\alpha}=\Delta^{-n} F_{\omega}(\xi)$ in the notation of (6.5.1). It follows from (6.6.2) that

$$
\bar{m}_{\Omega} F_{\alpha}=\bar{m}_{\omega} .
$$

Take $\lambda \in \Lambda^{+}$such that $\alpha_{\mathbf{p}}(\lambda)=\alpha$. Then $\mathfrak{t}^{\lambda} \in \operatorname{Std}(\lambda)_{\omega}$. Let $S_{\omega}=\omega\left(\mathfrak{t}^{\lambda}\right) \in \mathcal{T}_{0}^{\mathbf{p}}(\lambda, \omega)$. Then $\bar{m}_{S_{\omega} \mathfrak{t}^{\lambda}}=\bar{m}_{\mathfrak{t}^{\lambda} \mathfrak{t}^{\lambda}}=\bar{m}_{\lambda} \in \bar{M}^{\omega}$. Since $\bar{M}^{\omega}=\bar{m}_{\omega} \overline{\mathcal{H}}^{\mathbf{p}}$, there exists $y_{\lambda} \in \overline{\mathcal{H}}^{\mathbf{p}}$ such that $\bar{m}_{\lambda}=\bar{m}_{\omega} y_{\lambda}$. One can choose $y_{\lambda}$ in the following way. Let $\overline{\mathcal{H}}_{\alpha}^{0}$ be the subalgebra of $\overline{\mathcal{H}}^{\mathbf{p}}$ consisting of scalar matrices with entries in $\mathcal{H}_{\alpha}=\mathcal{H}_{n_{1}, r_{1}} \otimes \cdots \otimes \mathcal{H}_{n_{g}, r_{g}}$ under the isomorphism in Proposition 5.3. Thus $\overline{\mathcal{H}}_{\alpha}^{0} \simeq \mathcal{H}_{\alpha}$. We have $\bar{m}_{\omega}, \bar{m}_{\lambda} \in \bar{M}_{0}^{\omega}$, and under the isomorphism $\bar{M}_{0}^{\omega} \simeq M^{\omega^{[1]}} \otimes \cdots \otimes M^{\omega^{[g]}}=\mathcal{H}_{\alpha}, \bar{m}_{\omega}$ corresponds to $1 \otimes \cdots \otimes 1$, and $\bar{m}_{\lambda}$ corresponds to $m_{\lambda^{[1]}} \otimes \cdots \otimes m_{\lambda^{[g]}}$ in $\mathcal{H}_{\alpha}$. Then we choose $y_{\lambda} \in \overline{\mathcal{H}}_{\alpha}^{0}$ as the scalar matrix consisting of $m_{\lambda^{[1]}} \otimes \cdots \otimes m_{\lambda^{[g]}}$ in $\mathcal{H}_{\alpha}$ under the isomorphism in Proposition 5.3.

Note that $F_{\alpha}$ commutes with any element in $\overline{\mathcal{H}}_{\alpha}^{0}$. In fact, by (7.2.1) $F_{\alpha} \in$ $R\left[\xi_{1}, \ldots, \xi_{n}\right]^{\mathfrak{S}_{\alpha}}$ with $\mathfrak{S}_{\alpha}=\mathfrak{S}_{n_{1}} \times \cdots \times \mathfrak{S}_{n_{g}}$, and a similar argument as in [SawS, Lemma 2.8] can be applied. In particular, $y_{\lambda}$ commutes with $F_{\alpha}$. Let $*: \overline{\mathcal{H}}^{\mathbf{p}} \rightarrow \overline{\mathcal{H}}^{\mathbf{p}}$ be the anti-automorphism. Since $\xi_{i}$ are fixed by $*, F_{\alpha}$ is fixed by $*$. Also, $y_{\lambda}$ is fixed by $*$ since the corresponding elements in $\mathcal{H}_{n_{k}, r_{k}}$ are fixed by $*$. We have the following lemma. 
Lemma 7.3. For each $\mathfrak{t}, \mathfrak{s} \in \operatorname{Std}(\lambda)$, we have

$$
m_{\mathfrak{s t}}^{\mathbf{p}}=T_{d(\mathfrak{s})}^{*} F_{\alpha} y_{\lambda} T_{d(\mathfrak{t})} .
$$

Proof. By the construction in 7.2, we see that $\bar{m}_{\Omega} F_{\alpha} y_{\lambda}=\bar{m}_{t^{\lambda} \mathfrak{t}^{\lambda}}$ for $\lambda$ such that $\alpha_{\mathbf{p}}(\lambda)=\alpha$. Thus $\bar{m}_{\Omega} F_{\alpha} y_{\lambda} T_{d(\mathfrak{t})}=\bar{m}_{\mathfrak{t}^{\lambda} \mathfrak{t}}$ for any $\mathfrak{t} \in \operatorname{Std}(\lambda)$. If $T \in \mathcal{T}_{0}^{\mathbf{p}}\left(\lambda, \omega^{\prime}\right)$ corresponds to $\mathfrak{t} \in \operatorname{Std}(\lambda)$ under (5.1.2), and $S_{\omega} \in \mathcal{T}_{0}^{\mathbf{p}}(\lambda, \omega)$ with $\omega=\omega_{\alpha}$, then we have $\varphi_{S_{\omega} T}\left(\bar{m}_{\Omega}\right)=\bar{m}_{\mathfrak{t}^{\lambda} \mathfrak{t}}$. It follows that $\varphi_{S_{\omega} T}=F_{\alpha} y_{\lambda} T_{d(\mathfrak{t})}$. This shows that $\varphi_{S S_{\omega}}=$ $T_{d(\mathfrak{s})}^{*} F_{\alpha} y_{\lambda}$. Take $T \in \mathcal{T}_{0}^{\mathbf{p}}\left(\lambda, \omega^{\prime}\right)$ corresponding to $\mathfrak{t} \in \operatorname{Std}(\lambda)$. Since $\varphi_{S S_{\omega}}\left(\bar{m}_{\Omega}\right)=$ $\bar{m}_{\mathfrak{s t}}{ }^{\lambda}$, we have

$$
\bar{m}_{\Omega} \cdot T_{d(\mathfrak{s})}^{*} F_{\alpha} y_{\lambda} T_{d(\mathfrak{t})}=\bar{m}_{\mathfrak{s t} \mathfrak{\lambda}^{\lambda}} \cdot T_{d(\mathfrak{t})}=\bar{m}_{\mathfrak{s t}}=\varphi_{S T}\left(\bar{m}_{\Omega}\right) .
$$

Thus we have $m_{\mathfrak{s t}}^{\mathbf{p}}=\varphi_{S T}=T_{d(\mathfrak{s})}^{*} F_{\alpha} y_{\lambda} T_{d(\mathfrak{t})}$.

7.4. For each $\mu \in \Lambda$ such that $\alpha_{\mathbf{p}}(\mu)=\alpha$, we define $y_{\mu} \in \overline{\mathcal{H}}_{\alpha}^{\mathbf{p}}$ similarly as before, by extending the definition of $y_{\lambda}$ for $\lambda \in \Lambda^{+}$. We define a right ideal $M_{\mathbf{p}}^{\mu}$ of $\overline{\mathcal{H}}^{\mathbf{p}}$ by $M_{\mathbf{p}}^{\mu}=F_{\alpha} y_{\mu} \overline{\mathcal{H}}^{\mathbf{p}}$ and put $M_{\mathbf{p}}=\bigoplus_{\mu \in \Lambda} M_{\mathbf{p}}^{\mu}$. By Lemma 4.10, we have $\bar{m}_{\Omega} F_{\alpha} y_{\mu}=\bar{m}_{\mu}$ and so $\bar{m}_{\Omega} F_{\alpha} y_{\mu} \overline{\mathcal{H}}^{\mathbf{p}}=\bar{m}_{\mu} \overline{\mathcal{H}}^{\mathbf{p}}=\bar{M}^{\mu}$. This shows that there exists an isomorphism $\phi: M_{\mathbf{p}}^{\mu} \rightarrow \bar{M}^{\mu}$ of $\overline{\mathcal{H}}^{\mathbf{p}}$-modules by $F_{\alpha} y_{\mu} h \mapsto \bar{m}_{\Omega} F_{\alpha} y_{\mu} h=\bar{m}_{\mu} h$.

Recall that $\left\{\bar{m}_{S \mathfrak{t}} \mid S \in \mathcal{T}_{0}^{\mathbf{P}}(\lambda, \mu), \mathfrak{t} \in \operatorname{Std}(\lambda)\right.$ for $\left.\lambda \in \Lambda^{+}\right\}$gives a basis of $\bar{M}^{\mu}$. In connection with this, we define, for each $S \in \mathcal{T}_{0}^{\mathbf{p}}(\lambda, \mu), \mathfrak{t} \in \operatorname{Std}(\lambda)$ with $\lambda \in \Lambda^{+}$,

$$
m_{S \mathfrak{t}}^{\mathbf{p}}=\sum_{\substack{\mathfrak{s} \in \operatorname{Std}(\lambda) \\ \mu(\mathfrak{s})=S}} q^{l(d(\mathfrak{s}))+l(d(\mathfrak{t}))} m_{\mathfrak{s t}}^{\mathbf{p}} .
$$

The following lemma holds.

Lemma 7.5. The set $\left\{m_{S \mathfrak{t}}^{\mathbf{p}}\right\}$ gives rise to a basis of $M_{\mathbf{p}}^{\mu}$, and we have $\phi\left(m_{S \mathfrak{t}}^{\mathbf{p}}\right)=\bar{m}_{S t}$ for each basis element.

Proof. By the proof of Lemma 7.3, we know that $\bar{m}_{\Omega} m_{\mathfrak{s t}}^{\mathbf{p}}=\bar{m}_{\mathfrak{s t}}$ for any $\mathfrak{t}, \mathfrak{s} \in \operatorname{Std}(\lambda)$. It follows that $\bar{m}_{\Omega} m_{S \mathfrak{t}}^{\mathbf{p}}=\bar{m}_{S \mathfrak{t}} \in \bar{M}^{\mu}$ for any $S \in \mathcal{T}_{0}^{\mathbf{p}}(\lambda, \mu)$ and $\mathfrak{t} \in \operatorname{Std}(\lambda)$. In particular, we see that $m_{S \mathfrak{t}}^{\mathbf{p}} \in M_{\mathbf{p}}^{\mu}$, and the lemma follows.

The following result gives the Schur-Weyl duality, i.e., the double centralizer property between $\overline{\mathcal{H}}^{\mathbf{p}}$ and $\overline{\mathcal{S}}^{\mathbf{p}}$.

Theorem 7.6. Under the assumptions (5.1.1) and (6.3.1), there exist isomorphisms of R-algebras

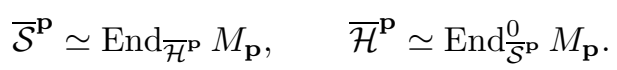

Proof. We argue on $\bar{M}$ instead of $M_{\mathbf{p}}$. The second isomorphism is already shown in (5.4.1). So we prove the first isomorphism. Let $\mu, \nu \in \Lambda$ be such that $\alpha_{\mathbf{p}}(\mu)=$ $\alpha_{\mathbf{p}}(\nu)=\alpha$, and take $\varphi \in \operatorname{Hom}_{\overline{\mathcal{H}}^{\mathbf{p}}}\left(\bar{M}^{\nu}, \bar{M}^{\mu}\right)$. Since $\bar{M}^{\nu}=\bar{m}_{\nu} \overline{\mathcal{H}}^{\mathbf{p}}$, the map $\varphi$ is determined by $\varphi\left(\bar{m}_{\nu}\right)$. We show that

$$
\varphi\left(\bar{m}_{\nu}\right) \in \bar{M}_{0}^{\mu} .
$$

In fact, since $\bar{m}_{S \mathfrak{t}}\left(S \in \mathcal{T}_{0}^{\mathbf{P}}(\lambda, \mu), \mathfrak{t} \in \operatorname{Std}(\lambda)\right)$ gives a basis of $\bar{M}^{\mu}$, one can write

$$
\varphi\left(\bar{m}_{\nu}\right)=\sum_{S, \mathfrak{t}} c_{S \mathrm{t}} \bar{m}_{S \mathrm{t}}
$$


with $c_{S t} \in R$. By (6.1.4), we have

$$
\varphi\left(\bar{m}_{\nu} \xi_{i}\right)=Q_{b(i)}^{\mathbf{p}} \sum_{S, \mathfrak{t}} c_{S \mathfrak{t}} \bar{m}_{S \mathfrak{t}}
$$

for $i=1, \ldots, n$, where $b(i)=k$ if $a_{k}+1 \leq i \leq a_{k}+n_{k}$. On the other hand, by (6.1.3), we have

$$
\varphi\left(\bar{m}_{\nu} \xi_{i}\right)=\varphi\left(\bar{m}_{\nu}\right) \xi_{i}=\sum_{S, \mathfrak{t}} c_{S \mathfrak{t}} Q_{\mathfrak{t}(i)}^{\mathbf{p}} \bar{m}_{S \mathfrak{t}}
$$

where $\mathfrak{t}(i)=k$ if the letter $i$ is contained in $\mathfrak{t}^{[k]}$ (see the remark after (6.1.3)). Comparing (7.6.2) and (7.6.3), we see that $\mathfrak{t} \in \operatorname{Std}(\lambda)_{0}$. Since $\bar{M}_{0}^{\mu}$ is spanned by $\bar{m}_{S \mathfrak{t}}$ with $\mathfrak{t} \in \operatorname{Std}(\lambda)_{0}$, we obtain (7.6.1).

Let $\overline{\mathcal{H}}_{\alpha}^{0}$ be the subalgebra of $\overline{\mathcal{H}}^{\mathbf{p}}$ generated by $T_{\alpha, i}^{[k]}$ for $k=1, \ldots, g, i=0, \ldots, n_{k}-$ 1. Since $\bar{M}_{0}^{\nu}=\bar{m}_{\nu} \overline{\mathcal{H}}_{\alpha}^{0}$, and similarly for $\bar{M}_{0}^{\mu}$, it follows from (7.6.1) that any $\varphi \in \operatorname{Hom}_{\overline{\mathcal{H}}^{\mathbf{p}}}\left(\bar{M}^{\nu}, \bar{M}^{\mu}\right)$ has the property that $\varphi\left(\bar{M}_{0}^{\nu}\right) \subset \bar{M}_{0}^{\mu}$. Thus we have a natural $R$-linear map

$$
\theta^{\prime \prime}: \operatorname{Hom}_{\overline{\mathcal{H}}^{\mathbf{p}}}\left(\bar{M}^{\nu}, \bar{M}^{\mu}\right) \rightarrow \operatorname{Hom}_{\overline{\mathcal{H}}_{\alpha}^{0}}\left(\bar{M}_{0}^{\nu}, \bar{M}_{0}^{\mu}\right),
$$

which is clearly injective. Let $\bar{H}_{\mu \nu}$ be the $\mu \nu$-part of $\bar{S}^{\mathbf{p}}$ as in (4.9.2). Since the action of $\widetilde{H}_{\alpha}$ on $\bar{M}_{0}^{\nu}$ coincides with the action of $\overline{\mathcal{H}}_{\alpha}^{0}$, we see that there exists an $R$-linear isomorphism

$$
\Theta: \bar{H}_{\mu \nu} \rightarrow \operatorname{Hom}_{\overline{\mathcal{H}}_{\alpha}^{0}}\left(\bar{M}_{0}^{\nu}, \bar{M}_{0}^{\mu}\right)
$$

by Lemma 4.12. On the other hand, by (5.4.2), we know that there exists an injective map $\theta^{\prime}: \bar{H}_{\mu \nu} \rightarrow \operatorname{Hom}_{\overline{\mathcal{H}}^{\mathrm{p}}}\left(\bar{M}^{\nu}, \bar{M}^{\mu}\right)$. It is clear that the composite of $\theta^{\prime}$ and $\theta^{\prime \prime}$ coincides with $\Theta$. Hence $\theta^{\prime}$ is an isomorphism. This shows that $\overline{\mathcal{S}}^{\mathbf{p}} \simeq \operatorname{End}_{\overline{\mathcal{H}}^{\mathbf{p}}} \bar{M}$, and the theorem follows.

Remark 7.7. The assumption (6.3.1) is used to give an expression of $\bar{M}$ as an ideal of $\overline{\mathcal{H}}^{\mathbf{p}}$. But the Schur-Weyl duality holds for $\bar{M}$ without referring to the ideal $M_{\mathbf{p}}$. In that case, (6.3.1) can be replaced by a weaker assumption "the parameters $Q_{1}^{\mathrm{p}}, \ldots, Q_{g}^{\mathbf{p}}$ are all distinct.".

By making use of Theorem 7.6, we obtain the following additional information on the space $\bar{H}_{\mu \nu}=\operatorname{Hom}_{\overline{\mathcal{H}}^{\mathbf{p}}}\left(M_{\mathbf{p}}^{\nu}, M_{\mathbf{p}}^{\mu}\right)$. Put $m_{\nu}^{\mathbf{p}}=F_{\alpha} y_{\nu}$ so that $M_{\mathbf{p}}^{\nu}=m_{\nu}^{\mathbf{p}} \overline{\mathcal{H}}^{\mathbf{p}}$.

Proposition 7.8. Let $\mu, \nu \in \Lambda$ such that $\alpha_{\mathbf{p}}(\mu)=\alpha_{\mathbf{p}}(\nu)=\alpha$.

(i) The map $\varphi \mapsto \varphi\left(m_{\nu}^{\mathbf{p}}\right)$ gives an isomorphism of $R$-modules,

$$
\operatorname{Hom}_{\overline{\mathcal{H}}^{\mathbf{p}}}\left(M_{\mathbf{p}}^{\nu}, M_{\mathbf{p}}^{\mu}\right) \rightarrow M_{\mathbf{p}}^{\nu *} \cap M_{\mathbf{p}}^{\mu},
$$

where $M_{\mathbf{p}}^{\nu *}=\overline{\mathcal{H}}^{\mathbf{p}} m_{\nu}^{\mathbf{p}}$ is the image of $M_{\mathbf{p}}^{\nu}$ under the operation $*$.

(ii) We have

$$
M_{\mathbf{p}}^{\nu *} \cap M_{\mathbf{p}}^{\mu}=F_{\alpha}\left(\overline{\mathcal{H}}_{\alpha}^{0} y_{\nu} \cap y_{\mu} \overline{\mathcal{H}}_{\alpha}^{0}\right)
$$

Proof. For each $m \in M_{\mathbf{p}}^{\nu *} \cap M_{\mathbf{p}}^{\mu}$, the map $m_{\nu}^{\mathbf{p}} h \mapsto m h\left(h \in \overline{\mathcal{H}}^{\mathbf{p}}\right)$ gives a welldefined map $\varphi_{m} \in \operatorname{Hom}_{\overline{\mathcal{H}}^{\mathbf{p}}}\left(M_{\mathbf{p}}^{\nu}, M_{\mathbf{p}}^{\mu}\right)$, and the map $m \mapsto \varphi_{m}$ gives an $R$-linear map $M_{\mathbf{p}}^{\nu *} \cap M_{\mathbf{p}}^{\mu} \rightarrow \operatorname{Hom}_{\overline{\mathcal{H}}^{\mathbf{p}}}\left(M_{\mathbf{p}}^{\nu}, M_{\mathbf{p}}^{\mu}\right)$, which is clearly injective. 
On the other hand, we have

$$
\begin{aligned}
\operatorname{Hom}_{\overline{\mathcal{H}}^{\mathbf{p}}}\left(M_{\mathbf{p}}^{\nu}, M_{\mathbf{p}}^{\mu}\right) & =\operatorname{Hom}_{\overline{\mathcal{H}}_{\alpha}^{\mathrm{p}}}\left(M_{\mathbf{p}}^{\nu}, M_{\mathbf{p}}^{\mu}\right) \\
& \simeq \operatorname{Hom}_{\overline{\mathcal{H}}_{\alpha}^{0}}\left(m_{\nu}^{\mathbf{p}} \overline{\mathcal{H}}_{\alpha}^{0}, m_{\mu}^{\mathbf{p}} \mathcal{H}_{\alpha}^{0}\right) \\
& \simeq \operatorname{Hom}_{\mathcal{H}_{\alpha}}\left(M^{\nu^{[1]}} \otimes \cdots \otimes M^{\nu^{[g]}}, M^{\mu^{[1]}} \otimes \cdots \otimes M^{\mu^{[g]}}\right),
\end{aligned}
$$

where $\mathcal{H}_{\alpha}=\mathcal{H}_{n_{1}, r_{1}} \otimes \cdots \otimes \mathcal{H}_{n_{g}, r_{g}}$. (Note that $m_{\mu}^{\mathbf{p}} \overline{\mathcal{H}}_{\alpha}^{0}$ corresponds to $\bar{M}_{0}^{\mu}$ under the isomorphism $M_{\mathbf{p}}^{\mu} \simeq \bar{M}^{\mu}$.) It is known, by [DJM] that the last set is isomorphic to $\overline{\mathcal{H}}_{\alpha}^{0} y_{\nu} \cap y_{\mu} \overline{\mathcal{H}}_{\alpha}^{0}$ as $R$-modules. Hence

$$
\operatorname{Hom}_{\overline{\mathcal{H}}_{\alpha}^{0}}\left(m_{\nu}^{\mathbf{p}} \overline{\mathcal{H}}_{\alpha}^{0}, m_{\mu}^{\mathbf{p}} \mathcal{H}_{\alpha}^{0}\right) \simeq\left(m_{\nu}^{\mathbf{p}} \overline{\mathcal{H}}_{\alpha}^{0}\right)^{*} \cap m_{\mu}^{\mathbf{p}} \overline{\mathcal{H}}_{\alpha}^{0}
$$

via the map $\varphi \mapsto \varphi\left(m_{\nu}^{\mathbf{p}}\right)$. But since $m_{\nu}^{\mathbf{p}} \overline{\mathcal{H}}_{\alpha}^{0}=F_{\alpha} y_{\nu} \overline{\mathcal{H}}_{\alpha}^{0}$ and $F_{\alpha}$ commutes with $y_{\nu}$ and $\overline{\mathcal{H}}_{\alpha}^{0}$, we see that $\left(m_{\nu}^{\mathbf{p}} \overline{\mathcal{H}}_{\alpha}^{0}\right)^{*}=F_{a} \overline{\mathcal{H}}_{\alpha}^{0} y_{\nu}$. This shows that

$$
\operatorname{Hom}_{\overline{\mathcal{H}^{\mathbf{p}}}}\left(M_{\nu}^{\mathbf{p}}, M_{\mu}^{\mathbf{p}}\right) \simeq F_{\alpha}\left(\overline{\mathcal{H}}_{\alpha}^{0} y_{\nu} \cap y_{\mu} \overline{\mathcal{H}}_{\alpha}^{0}\right) \subseteq M_{\mathbf{p}}^{\nu *} \cap M_{\mathbf{p}}^{\mu},
$$

where the first isomorphism is given by the map $\varphi \mapsto \varphi\left(m_{\nu}^{\mathbf{p}}\right)$. Both statements of the proposition follow from this, by being combined with the remark in the first paragraph.

\section{Comparison of $\overline{\mathcal{H}}^{\mathbf{p}}$ FOR various $\mathbf{p}$}

8.1. We shall consider the relationship among $\mathcal{S}^{\mathbf{p}}$ and $\overline{\mathcal{H}}^{\mathbf{p}}$ for various types $\mathbf{p}$. First consider the case of $\mathcal{S}^{\mathbf{p}}$. Let $\mathbf{p}=\left(r_{1}, \ldots, r_{g}\right)$ and let $\mathbf{p}^{\prime}=\left(r_{1}^{\prime}, \ldots, r_{g^{\prime}}^{\prime}\right)$ be two compositions of $r$. We define $\left(p_{1}^{\prime}, \ldots, p_{g^{\prime}}^{\prime}\right)$ for $\mathbf{p}^{\prime}$ similar to $\mathbf{p}$. We write $\mathbf{p}^{\prime} \preceq \mathbf{p}$ if $\mathbf{p}^{\prime}$ is obtained as a refinement of $\mathbf{p}$, namely if $p_{j}$ coincides with some $p_{k_{j}}^{\prime}$ for each $j$. In particular, we have $\left(1^{r}\right) \preceq \mathbf{p} \preceq(r)$ for any $\mathbf{p}$. Assume that $\mathbf{p}^{\prime} \preceq \mathbf{p}$. Then we see that $\mathbf{a}_{\mathbf{p}}(\lambda) \geq \mathbf{a}_{\mathbf{p}}(\mu)$ if $\mathbf{a}_{\mathbf{p}^{\prime}}(\lambda) \geq \mathbf{a}_{\mathbf{p}^{\prime}}(\mu)$ for $\lambda, \mu \in \Lambda$. Moreover, $\alpha_{\mathbf{p}}(\lambda)=\alpha_{\mathbf{p}}(\mu)$ if $\alpha_{\mathbf{p}^{\prime}}(\lambda)=\alpha_{\mathbf{p}^{\prime}}(\mu)$. This implies that

$$
\mathcal{S}^{\mathbf{p}^{\prime}} \subseteq \mathcal{S}^{\mathbf{p}} \quad \text { if } \mathbf{p}^{\prime} \preceq \mathbf{p} .
$$

Concerning the modified Ariki-Koike algebras $\overline{\mathcal{H}}^{\mathbf{p}}$, we have the following.

Proposition 8.2. There exists an algebra homomorphism $\rho_{\mathbf{p}^{\prime} \mathbf{p}}: \overline{\mathcal{H}}^{\mathbf{p}} \rightarrow \overline{\mathcal{H}}^{\mathbf{p}^{\prime}}$ for any pair $\mathbf{p}, \mathbf{p}^{\prime}$ such that $\mathbf{p}^{\prime} \preceq \mathbf{p}$ satisfying the following property: for $\mathbf{p}^{\prime \prime} \preceq \mathbf{p}^{\prime} \preceq \mathbf{p}$, we have $\rho_{\mathbf{p}^{\prime \prime} \mathbf{p}}=\rho_{\mathbf{p}^{\prime \prime} \mathbf{p}^{\prime}} \circ \rho_{\mathbf{p}^{\prime} \mathbf{p}}$.

Proof. Let $\bar{M}=\bigoplus_{\mu} \bar{M}^{\mu}$ be the $\mathcal{H}$-module defined by $\widehat{N}^{\mathbf{a}_{\mathbf{p}}(\mu)}$ as before. We denote $\bar{M}$ by $\bar{M}_{\mathbf{p}}$ to indicate its dependence on $\mathbf{p}$. Assume that $\mathbf{p}^{\prime} \preceq \mathbf{p}$. Then we have a natural surjection $\bar{M}_{\mathbf{p}} \rightarrow \bar{M}_{\mathbf{p}^{\prime}}$ of $\mathcal{H}$-modules. If we regard $\bar{M}_{\mathbf{p}}$ as a left $\mathcal{S}^{\mathbf{p}}$-module, and $\bar{M}_{\mathbf{p}^{\prime}}$ as a left $\mathcal{S}^{\mathbf{p}^{\prime}}$-module, then the map $\bar{M}_{\mathbf{p}} \rightarrow \bar{M}_{\mathbf{p}^{\prime}}$ is compatible with the actions of $\mathcal{S}^{\mathbf{p}}$ and $\mathcal{S}^{\mathbf{p}^{\prime}}$ via the inclusion $\mathcal{S}^{\mathbf{p}^{\prime}} \hookrightarrow \mathcal{S}^{\mathbf{p}}$. Let $M_{\mathbf{p}}=\bigoplus_{\mu} M_{\mathbf{p}}^{\mu}$ be as in 7.4. By Theorem 7.6, $\overline{\mathcal{H}}^{\mathbf{p}}$ is realized as $\overline{\mathcal{H}}^{\mathbf{p}}=\operatorname{End} \frac{0}{\mathcal{S}^{\mathbf{p}}} M_{\mathbf{p}}$. By using the property of the cellular structure of $\overline{\mathcal{H}}^{\mathbf{p}}$ described in the beginning of Section 7, together with Lemma 7.5, the above property of $\bar{M}_{\mathbf{p}}$ can be made more precise for $M_{\mathbf{p}}$ as follows (which is a generalization of the argument in 4.1). Let $\widehat{N}_{\mathbf{p}}^{\mathbf{a}^{\prime}(\mu)}$ be the $R$-submodule of $\overline{\mathcal{H}}^{\mathbf{p}}$ spanned by $m_{\mathfrak{s t}}^{\mathbf{p}}$ such that $\mathfrak{s}, \mathfrak{t} \in \operatorname{Std}(\lambda)$ with $\mathbf{a}_{\mathbf{p}^{\prime}}(\lambda)>\mathbf{a}_{\mathbf{p}^{\prime}}(\mu)$. Then $\widehat{N}_{\mathbf{p}}^{\mathbf{a}_{\mathbf{p}^{\prime}}(\mu)}$ 
is a two-sided ideal of $\overline{\mathcal{H}}^{\mathbf{p}}$. Put $\widehat{M}_{\mathbf{p}}^{\mu}=M_{\mathbf{p}}^{\mu} \cap N_{\mathbf{p}}^{\mathbf{a}^{\mathbf{p}^{\prime}}(\mu)}$. Then $\widehat{M}_{\mathbf{p}}^{\mu}$ is an $\overline{\mathcal{H}}^{\mathbf{p}}$-submodule of $M_{\mathbf{p}}^{\mu}$ with the basis $\left\{m_{S \mathfrak{t}}^{\mathbf{p}} \mid S \in \mathcal{T}_{0}^{\mathbf{P}}(\lambda, \mu), \mathfrak{t} \in \operatorname{Std}(\lambda), \mathbf{a}_{\mathbf{p}^{\prime}}(\lambda)>\mathbf{a}_{\mathbf{p}^{\prime}}(\mu)\right\}$, and we have an isomorphism of $R$-modules

$$
M_{\mathbf{p}}^{\mu} / \widehat{M}_{\mathbf{p}}^{\mu} \simeq M_{\mathbf{p}^{\prime}}^{\mu}
$$

A similar argument as in 4.9 shows that any $\varphi \in \bar{H}_{\mu \nu}$ maps $\widehat{M}^{\nu}$ to $\widehat{M}^{\mu}$, and so $\varphi \in \overline{\mathcal{S}}^{\mathbf{p}}=\operatorname{End}_{\overline{\mathcal{H}}^{\mathbf{p}}} M_{\mathbf{p}}$ induces an action on $M_{\mathbf{p}} / \widehat{M}_{\mathbf{p}}$, where $\widehat{M}_{\mathbf{p}}=\bigoplus_{\mu} \widehat{M}_{\mathbf{p}}^{\mu}$. This gives an isomorphism $M_{\mathbf{p}} / \widehat{M}_{\mathbf{p}} \simeq M_{\mathbf{p}^{\prime}}$ as $\overline{\mathcal{S}}^{\mathbf{p}^{\prime}}$-modules.

Now the action of $\overline{\mathcal{H}}^{\mathbf{p}}$ on $M_{\mathbf{p}}$ induces an action on $M_{\mathbf{p}} / \widehat{M}_{\mathbf{p}}$, which is compatible with the action of $\overline{\mathcal{S}}^{\mathbf{p}}$. Hence this induces an action of $\overline{\mathcal{H}}^{\mathbf{p}}$ on $M_{\mathbf{p}^{\prime}}$ compatible with the action of $\overline{\mathcal{S}}^{\mathbf{p}^{\prime}}$. Thus we have an $R$-algebra homomorphism

$$
\rho_{\mathbf{p}^{\prime} \mathbf{p}}: \overline{\mathcal{H}}^{\mathbf{p}} \rightarrow \operatorname{End}_{\overline{\mathcal{S}}^{\mathbf{p}^{\prime}}}^{0} M_{\mathbf{p}^{\prime}} \simeq \overline{\mathcal{H}}^{\mathbf{p}^{\prime}} .
$$

It is clear that this map $\rho_{\mathbf{p}^{\prime} \mathbf{p}}$ satisfies the required property.

8.3. In the case where $\mathbf{p}=(r)$, we have $\overline{\mathcal{H}}^{\mathbf{p}} \simeq \mathcal{H}$, and in the case where $\mathbf{p}^{\prime}=\left(1^{r}\right)$, we have $\overline{\mathcal{H}}^{\mathbf{p}^{\prime}} \simeq \overline{\mathcal{H}}$, the modified Ariki-Koike algebra introduced in SawS. We have $\mathbf{p}^{\prime} \preceq \mathbf{p}$, and the map $\rho_{\mathbf{p}^{\prime} \mathbf{p}}: \mathcal{H} \rightarrow \overline{\mathcal{H}}$ coincides with the map $\rho_{0}$ given in SawS, Lemma 1.5]. We consider the following separation condition on parameters of $\mathcal{H}$, which was first introduced in $\mathrm{A}$.

(8.3.1) $q^{2 k} Q_{i}-Q_{j} \in R$ are invertible in $R$ for $|k|<n, i \neq j$.

Note that the condition (8.3.1) is stronger than the condition (6.3.1) for any $\mathbf{p}$. It is shown by [SawS, 8.3.2], based on the result in [HS, that $\rho_{0}: \mathcal{H} \rightarrow \overline{\mathcal{H}}$ gives an isomorphism if the separation condition (8.3.1) holds. We have the following corollary.

Corollary 8.4. Suppose that the condition (8.3.1) holds for $\mathcal{H}$. Then $\mathcal{H} \simeq \overline{\mathcal{H}}^{\mathbf{p}}$ for any $\mathbf{p}$. In particular, Theorem 6.8 gives a new presentation for the Ariki-Koike algebra $\mathcal{H}$.

Proof. We have $\left(1^{r}\right) \preceq \mathbf{p} \preceq(r)$ for any $\mathbf{p}$. Since $\rho_{0}: \mathcal{H} \rightarrow \overline{\mathcal{H}}$ is an isomorphism, the map $\rho_{\mathbf{p}^{\prime} \mathbf{p}}: \overline{\mathcal{H}}^{\mathbf{p}} \rightarrow \overline{\mathcal{H}}^{\mathbf{p}^{\prime}} \simeq \mathcal{H}$ is surjective by Proposition 8.2 for $\mathbf{p}^{\prime}=\left(1^{r}\right)$. Since both $\mathcal{H}$ and $\overline{\mathcal{H}}^{\mathbf{p}}$ are free $R$-modules of the same rank, we obtain $\overline{\mathcal{H}}^{\mathbf{p}} \simeq \mathcal{H}$ as asserted.

\section{REFERENCES}

[A] S. Ariki, Cyclotomic $q$-Schur algebras as quotients of quantum algebras, J. Reine Angew. Math. 513 (1999), 53-69. MR.1713319 (2001a:16065)

[DJM] R. Dipper, G. James and A. Mathas, Cyclotomic $q$-Schur algebras, Math. Z. 229, (1998), 385-416. MR:1658581 (2000a:20033)

[DR] J. Du and H. Rui, Based algebras and standard bases for quasi-hereditary algebras, Trans. Amer. Math. Soc. 350 (1998), 3207-3235. MR1603902 (99b:16027)

[GL] J.J. Graham and G.I. Lehrer, Cellular algebras, Invent. Math., 123 (1996), 1-34. MR 1376244 (97h:20016)

[HS] J. Hu and F. Stoll, On double centralizer properties between quantum groups and ArikiKoike algebras, J. Algebra 275 (2004), no. 1, 397-418. MR2047454 (2005f:20012)

[JM] G.D. James and A. Mathas, The Jantzen sum formula for cyclotomic $q$-Schur algebras, Trans Amer. Math. Soc. 352 (2000), 5381-5404. MR1665333 (2001b:16017) 
[M] A. Mathas, The representation theory of the Ariki-Koike and cyclotomic $q$-Schur algebras, in "Representation Theory of Algebraic Groups and Quantum Groups", Adv. Stud. in Pure Math., 40 (2004), pp. 261-320. MR 2074597 (2005f:20014)

[Sa] N. Sawada, On decomposition numbers of the cyclotomic $q$-Schur algebras, J. Algebra 311 (2007), no. 1, 147-177. MR2309882 (2008c:20007)

[Sh] T. Shoji, A Frobenius formula for the characters of Ariki-Koike algebras. J. Algebra 226, (2000), 818-856. MR:1752762 (2001f:20013)

[SakS] M. Sakamoto and T. Shoji, Schur-Weyl reciprocity for Ariki-Koike algebras, J. Algebra 221 (1999), 293-314. MR.1722914 (2001f:17030)

[SawS] N. Sawada and T. Shoji, Modified Ariki-Koike algebras and cyclotomic $q$-Schur algebras, Math. Z. 249 (2005), 829-867. MR2126219 (2005j:20006)

[W] K. Wada, On decomposition numbers with Jantzen filtration of cyclotomic $q$-Schur algebras, to appear in Representation Theory, 2010.

Graduate School of Mathematics, Nagoya University, Chikusa-Ku, Nagoya 464-8602, JAPAN

Graduate School of Mathematics, Nagoya University, Chikusa-ku, Nagoya 464-8602, JAPAN

Current address: Research Institute for Mathematical Sciences, Kyoto University, Kyoto 6068502 Japan 